University of Louisville

ThinkIR: The University of Louisville's Institutional Repository

Electronic Theses and Dissertations

$12-2018$

\title{
Present but not accounted for: a phenomenological study of gifted African American males.
}

Ronda Elizabeth George
University of Louisville

Follow this and additional works at: https://ir.library.louisville.edu/etd

Part of the Bilingual, Multilingual, and Multicultural Education Commons, Gifted Education Commons, and the Junior High, Intermediate, Middle School Education and Teaching Commons

\section{Recommended Citation}

George, Ronda Elizabeth, "Present but not accounted for: a phenomenological study of gifted African American males." (2018). Electronic Theses and Dissertations. Paper 3103.

https://doi.org/10.18297/etd/3103

This Doctoral Dissertation is brought to you for free and open access by ThinkIR: The University of Louisville's Institutional Repository. It has been accepted for inclusion in Electronic Theses and Dissertations by an authorized administrator of ThinkIR: The University of Louisville's Institutional Repository. This title appears here courtesy of the author, who has retained all other copyrights. For more information, please contact thinkir@louisville.edu. 
PRESENT BUT NOT ACCOUNTED FOR: A PHENOMENOLOGICAL STUDY OF GIFTED AFRICAN AMERICAN MALES

\author{
By \\ Ronda Elizabeth George \\ B.A., University of Louisville, 1995 \\ M.A., Western Kentucky University, 2002
}

\begin{abstract}
A Dissertation Submitted to the Faculty of the College of Education and Human Development of the University of Louisville in Partial Fulfillment of the Requirements for the Degree of
\end{abstract}

Doctor of Education in

Educational Leadership and Organizational Development

Department of Evaluation, Leadership, and Organizational Development University of Louisville

Louisville, KY

December 2018 

PRESENT BUT NOT ACCOUNTED FOR: A PHENOMENOLOGICAL STUDY OF GIFTED AFRICAN AMERICAN MALES

By

Ronda Elizabeth George

B.A., University of Louisville, 1995

M.A., Western Kentucky University, 2002

A Dissertation Approved on

November 12, 2018

by the following Dissertation Committee

William Kyle Ingle, Ph.D.

Dissertation Co-Chair

Detra DeVerne Johnson, Ph.D.

Dissertation Co-Director

Mary Brydon-Miller, Ph.D.

Professor

Derrick R. Brooms, Ph.D.

Associate Professor 


\section{DEDICATION}

For my parents, Rev. Darwin Scrubb and Dr. Monica Scrubb and my brother Jason

Scrubb, in sincere appreciation for your unconditional and unwavering love, support, and encouragement. I am blessed beyond measure to have you in my life. 


\section{ACKNOWLEDGEMENTS}

"The Lord will accomplish that which concerns me; your lovingkindness, O Lord, endures forever...” (Psalm 138:8, AMP).

I would like to give honor to the Sovereign God, who is the head of my life, for the determination, strength, and courage to dream and the faith to see those dreams come true. My life is orchestrated by God and I am thankful that He has ordered my steps and his will is continually being accomplished in my life. I thank my parents, the most amazing people I know. You have been the wind beneath my wings, encouraging me to give my best, do my best, and be my best. Your prayers and words of encouragement have carried me through this journey, without which I would not have made it. Preparing

meals, running errands, and completing household chores for me so that I would not have to be concerned with trivialities, but work on the work, is a testament of your support and selflessness. Thank you for listening to doctoral student chatter (sometimes positive and sometimes in the form of complaining), being my audience when practicing for an important presentation, and giving valuable feedback to improve the substance and quality of my work. Thank you for leading by example and being life-long learners and leaders; my inspiration for being the same. I could not have asked for better parents. You have been to me what every child wants, needs, and aspires to become. Thank you, Jason, for being a wonderful brother and encouraging me to push beyond my own limits. 


\section{ABSTRACT \\ PRESENT BUT NOT ACCOUNTED FOR: A PHENOMENOLOGICAL STUDY OF GIFTED AFRICAN AMERICAN MALES \\ Ronda E. George}

November 12, 2018

The purpose of this research study was to explore the lived experiences of gifted African American males in grades 6-8. Specifically, the study examined how gifted African American males perceived and understood their participation in a gifted program and how their sense of self-efficacy shaped their disposition and approach toward academic persistence. Much of the literature published on the social, emotional, and academic success of gifted African American males has focused on college-aged students, therefore the current study is crucial in contributing to the body of knowledge on African American males, in particular, those identified as gifted during early adolescence (Ford, 2005). Additionally, voices of gifted African American males have been disregarded, yet are quintessential to understanding how they create meaning from their experiences.

This study utilized interpretive phenomenological analysis as a means of synthesizing and making sense from the themes which emerged in the study. Analysis of the data indicated that gifted African American males found the issue of underrepresentation and relationships with teachers and peers to have the most influence 
in understanding their experiences in a gifted program. The findings also revealed that scholar identity and the shaping of self-efficacy, driven by a need to succeed, influenced their academic persistence. The findings discussed give insight into the challenges and triumphs of gifted African American males participating in a gifted program and provide implications for policy and practice. 
TABLE OF CONTENTS

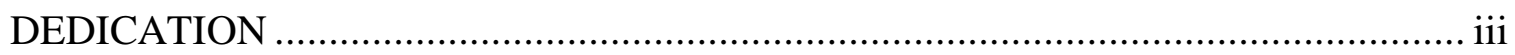

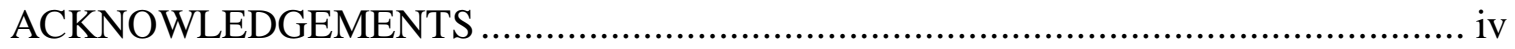

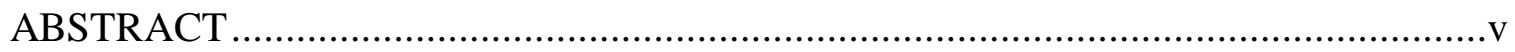

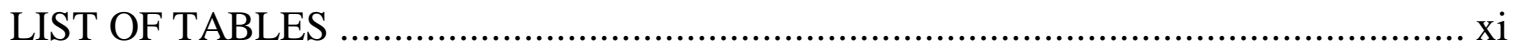

LIST OF FIGURES ...................................................................................... xii

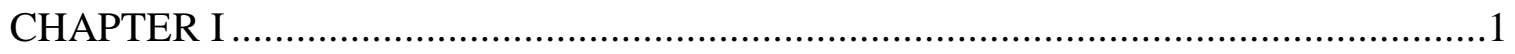

INTRODUCTION ..............................................................................................

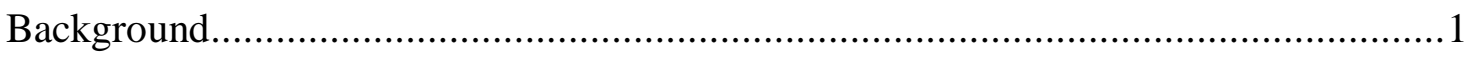

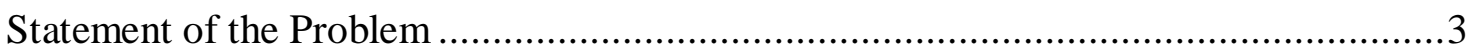

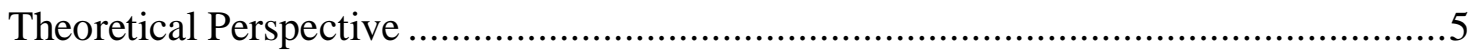

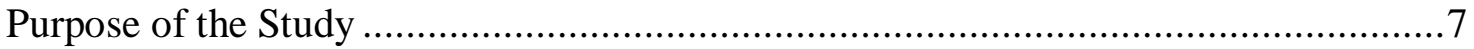

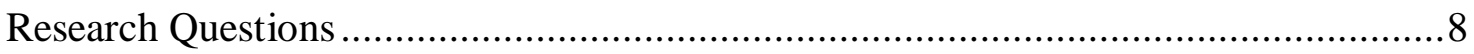

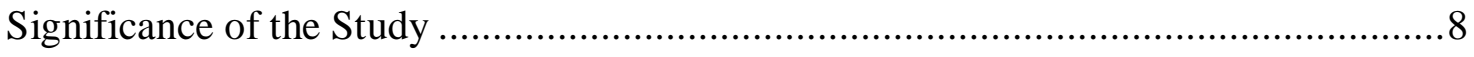

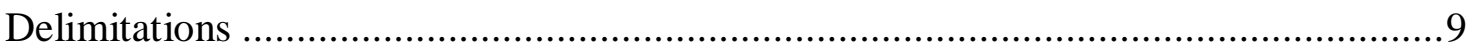

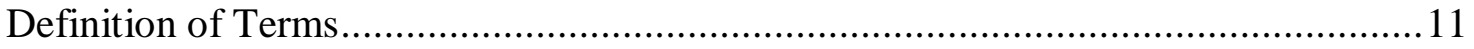

Organization of Chapters....................................................................................13 


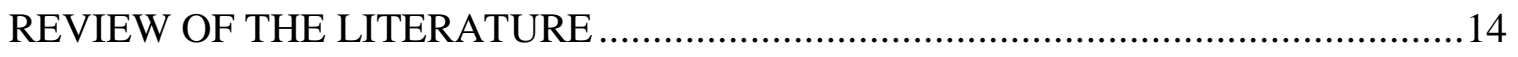

History of Gifted Programs in the United States ................................................. 16

Benefits of Gifted Programs in the United States................................................20

Trends in Gifted Enrollment in the United States...............................................27

Barriers to African American Enrollment in Gifted Programs..................................30

Barriers to African American Male Enrollment in Gifted Programs..........................39

Self-Efficacy and Academic Achievement for African American Males in Gifted

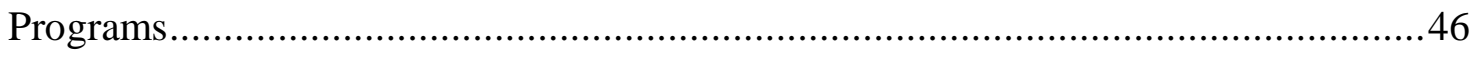

Critical Race Theory and its Application to African American Male Enrollment in

Gifted Programs ..................................................................................... 50

Group and Deficit Thinking Theory and its Application to African American Male

Enrollment in Gifted Programs.....................................................................52

Summary of the Literature Review Findings ..................................................55

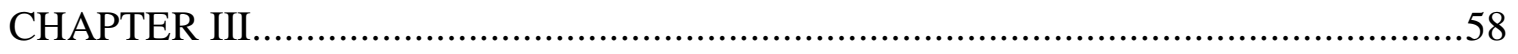

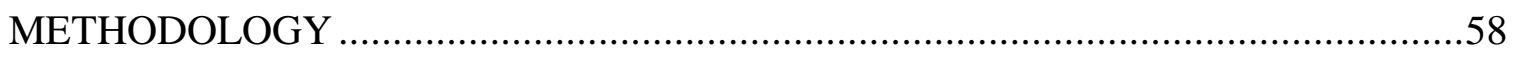

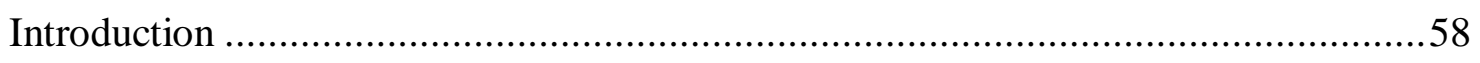

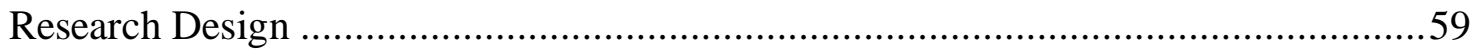

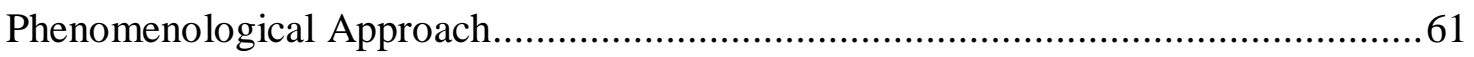

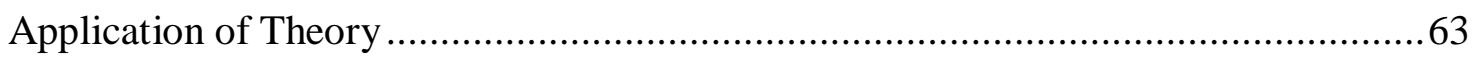

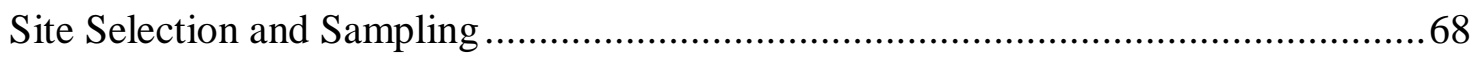


Data Collection Methods.

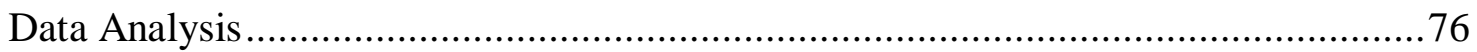

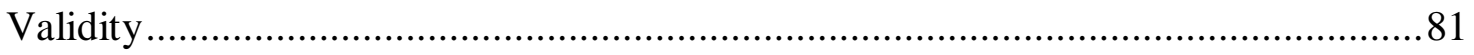

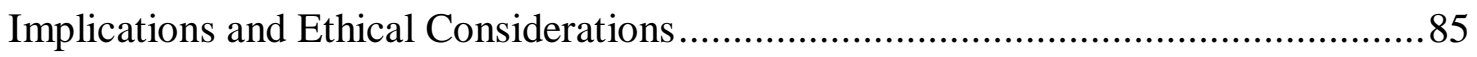

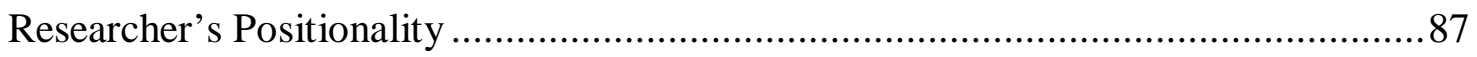

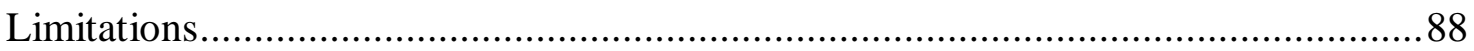

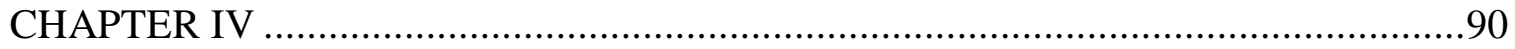

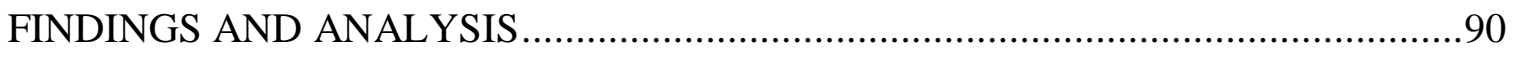

Contextual Description of the Gifted Program......................................................91

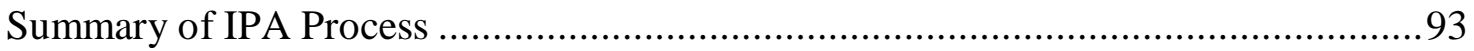

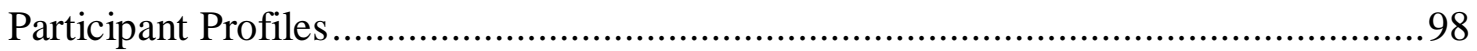

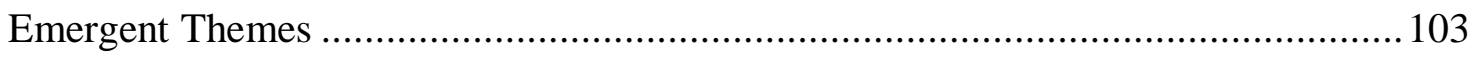

Experiences of Gifted African American Males ................................................. 107

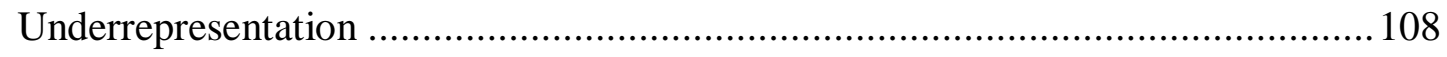

Teacher-Student Relationships............................................................... 114

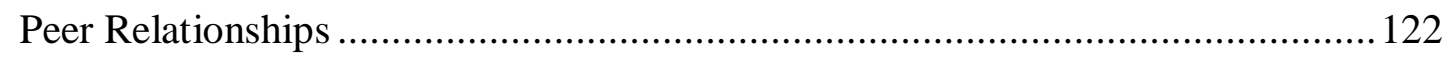

Self-Efficacy and Academic Persistence......................................................... 128

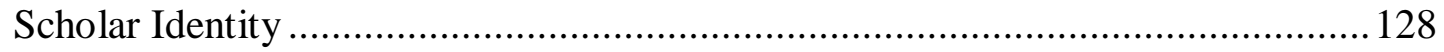

Self-Efficacy: The Need to Succeed.......................................................... 131

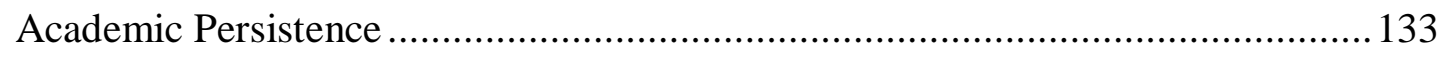

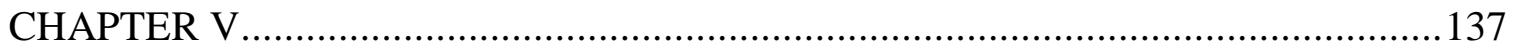

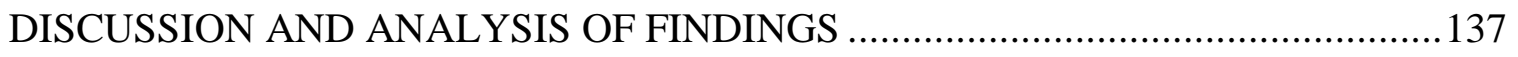

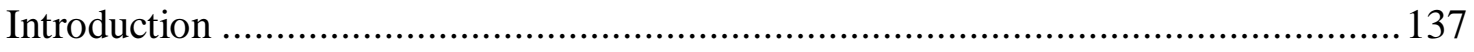

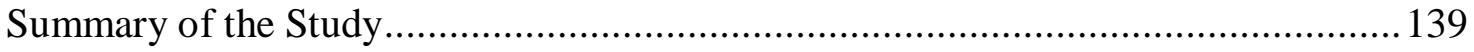


Discussion of the Findings \& Literature 144

Implications for Action and Practice .161

Recommendations for Future Research .166

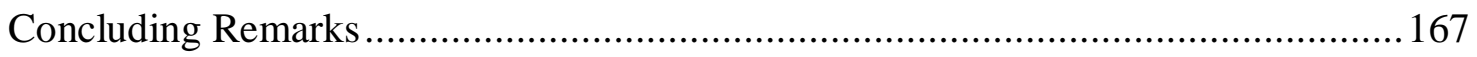

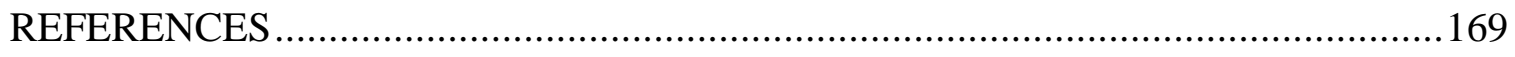

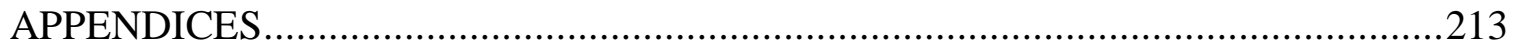

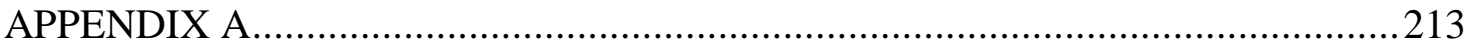

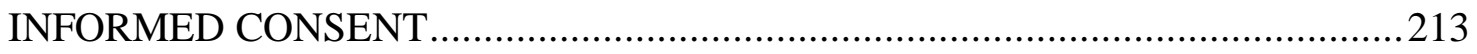

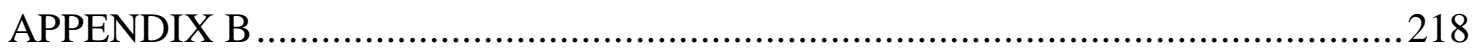

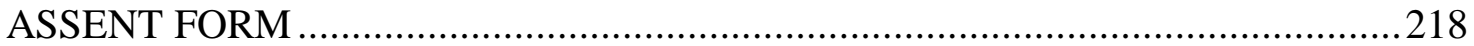

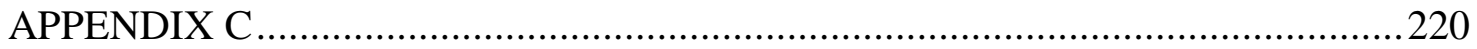

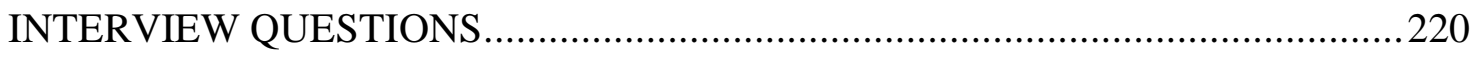

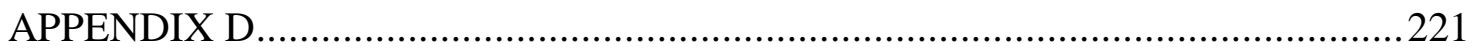

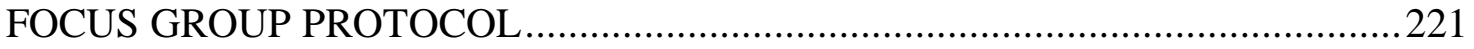

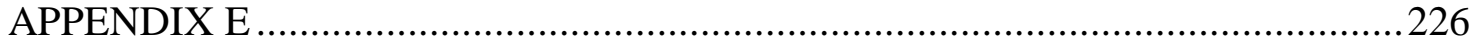

PHOTO ELICITATION IMAGES ................................................................. 226

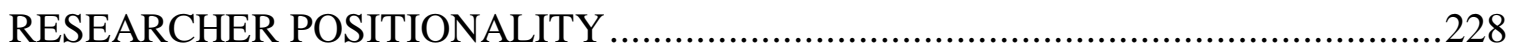

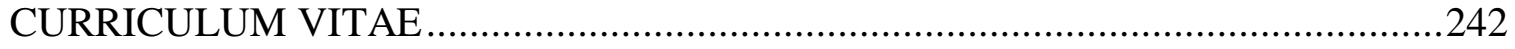


LIST OF TABLES

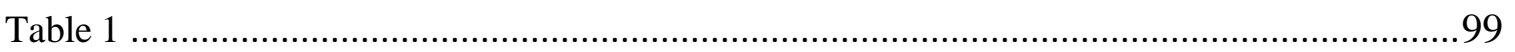




\section{LIST OF FIGURES}

Figure 1. Coding template used for semi-structured interviews. ..............................95

Figure 2. In vivo and focused coding for focus group discussion and photo elicitation...97

Figure 3. Emergent themes and subordinate themes......................................... 104

Figure 4. Participant's illustration of underrepresentation in a gifted program. ............109

Figure 5. Teacher's treatment perceived to be racially discriminatory and unfair. ........115

Figure 6. Participant's illustration depicting invisibility................................... 120 


\section{CHAPTER I}

\section{INTRODUCTION}

\section{Background}

The educational narratives of African American males are familiar recitations reflecting a systemic patchwork of economic, social, and academic inequities. African American males make up only 7\% of the United States (U.S.) student population, yet they are marginalized academically and socially more than any other minority group (Noguera, 2008; Schott Foundation, 2010). African American males are three times more likely to receive expulsions or suspensions from school than their White counterparts (Howard, 2013). They account for almost $80 \%$ of all students in special education programs, $20 \%$ of all students classified as mentally retarded, and less than $50 \%$ graduate from high school within four years, compared with $78 \%$ of their White peers (Schott Foundation for Public Education, 2010; U.S. Department of Education, 2010). Additionally, the internalization of pejorative images imposed upon African American males has profound latent effects as a result of crippling educational and social rhetoric (Howard \& Flennaugh, 2011). Furthermore, globalized characterizations that fail to probe the intersectionality of race and gender have profound implications on the educational experiences and academic outcomes of African American males (Howard, 2013). These issues are exacerbated for students who are African American, male, and gifted in particular, because their "success is less optimal when economic, cultural, 
socioemotional, affective, and developmental needs are ignored, trivialized, or poorly addressed” (Stambaugh \& Ford, 2015, p. 192).

The academic prognosis of gifted African American males continues to be unfavorable because of deficit ideologies that promote and perpetuate inequitable practices. Multiple studies have explored the underrepresentation of African American students in gifted programs (Anquiano, 2003; Harris, Brown, Ford, \& Richardson, 2003; Kearns, Ford, \& Linney, 2005). The studies examined the factors that influence the disproportionality in participation among African Americans in gifted programs from which inferences regarding the participation of gifted African males have been drawn. Although limited access to gifted programming, identification and retention practices, assessment instruments, culturally responsive pedagogy, and policy issues have collectively been an impediment to African American males’ participation in gifted programs, the most consequential barrier is the deficit orientation engrained in society and in schools (Harris, Brown, Ford, \& Richardson, 2004). African American males are 2.5 times less likely to be enrolled in gifted and talented programs, even if their prior academic performance is evidentiary of their ability to succeed (Schott Foundation for Public Education, 2010). In contrast, African American males often intentionally evade being labeled as gifted for fear of being taunted and accused of acting White or not being Black enough if it is perceived that their academic behaviors align with stereotypes associated with African American students (Fryer \& Torelli, 2010; Graham \& Anderson, 2008; Henfield, Moore, \& Wood, 2008). The stress produced from being stigmatized not only mitigates the prospect of enrollment, but once enrolled, gifted African American males disengage and elect to separate from gifted programs (Carpenter \& Ramirez, 2007; 
Fisher, 2005; Henfield, Washington, \& Owens, 2010; Mickelson \& Greene, 2006; National Center for Educational Statistics [NCES], 2011). Ford's (1992) seminal study indicated that feelings of loneliness and fear of rejection were prevalent among gifted African American males and contributed to underachievement. This is noteworthy because many African American students place high value on and thrive in communal settings (Ford, 2011).

Despite the alarming disproportionality of African American male participation in gifted programs, resilient African American males manage to persist socially, emotionally, and academically in gifted programs. Their counter narratives invalidate subversive stereotypes. The proliferation of such counter narratives hinges on establishing and maintaining academic environments in which gifted African American males find and develop their racial identity, self-esteem, and sense of self-efficacy (Hébert, 2002). Whiting (2006), building upon Bandura’s (1977, 1986) theory on selfefficacy, further establishes self-efficacy as the linchpin in the development of one's identity, specifically scholar identity. Gifted African American males who possess a well-developed scholarly identity perceive themselves to be academically confident, erudite, and creative (Whiting, 2009b). Thus, the development of scholar identity provides the tools for gifted African American males to articulate their lived experiences poignantly while participating in a gifted program.

\section{Statement of the Problem}

African American students, especially African American males, are underperforming in U.S. schools. They have the highest dropout rates, consistently lower 
test scores and grades, and are referred for special education services more than any other ethnic group (NCES, 2011). Palmer and Maramba (2011) reported that when African American males advance through school, they exhibit signs of disengagement, are more affected by peer relationships, school culture, school policies, and their academic performance is significantly lower than African American females (Palmer, Davis, \& Hilton, 2009). Howard (2013) asserted that "although research based on Black males has been informative and expansive over the last several decades, there still remains more to be studied, analyzed, and learned about the diversity of their experiences” (p. 80). In fact, Brooms (2015) contended that there is a need to capture the voices of Black males with regard to their perceptions and experiences regarding school, through the lens of their social and cultural experiences, as a means of evaluating how they make meaning of school.

With attention given to diagnosing and improving the circumstances which contribute to the poor academic performance of African American students as a group, far less attention is given to examining the needs of high-performing and gifted African American students in particular (Ford, 2011). The needs and characteristics of gifted African American students transcends the traditional needs often studied and applied in gifted populations, therefore their socio-emotional, academic, and psychological needs are overwhelmingly disregarded (Scott, 2012). This imbalance in research confirms the need to unveil previously dismissed truths regarding the untapped potential of gifted African American males participating in gifted programs and to reverse the narratives that have consistently undermined their academic contributions. 


\section{Theoretical Perspective}

In this study, Critical Race Theory (CRT) provides a lens through which to understand the experiences of gifted African American males. CRT evolved from the work of critical legal theorists such as Derrick Bell, Patricia Williams, Randall Kennedy, and Lani Guinier, who held that the historical function of legal theory served as a vehicle to perpetuate oppression and discrimination (Parker, 2008). Based on the universality of education, the centrality of race in education, and the prominence of discrimination within the context of education, CRT scholars (Delgado, 2000; Ladson-Billings, 1995, 2006; Parker, 2008; Solóranzo, 1997) argued in support of legal, social, and educational policy evaluation to be interrogated through the lens of race (Darder, 2011). Thus, CRT has become essential in postulating and interpreting the conditions influencing marginalized student populations in U.S. schools of which gifted African American males are a part. The use of CRT is equally appropriate for this phenomenological study because of the emphasis it places on 'experiential knowledge', recognizing the experiences of people of color to be legitimate and foundational to understanding and the influences of racial oppression (Barnes, 1990, p. 11; Delgado, 1995; LadsonBilling,1999; Soloranzo, 1997). Therefore, the nature of CRT in extracting narrative "has become successful among groups committed to making the voice of the voiceless heard in the public arena” (Viotti da Costa, 2001, p.21).

There remains a gross underrepresentation of minorities in general, and gifted African American males in particular, in gifted programs (Ford, 2011). CRT provides a means of interpreting underrepresentation as a function of race and racism that limit culturally different students, and in some instances, blatantly prevent access to 
educational programs to which they have a right. Restricted access to gifted programs is not a new phenomenon. Using CRT, Ladson-Billings and Tate (1995) analyzed educational inequity, finding that access to high quality and rigorous curriculum has primarily been the exclusive privilege of White students. Undertones of racism serve as the reason why CRT theorists contend that the consequences of racism are elevated when school texts and curriculum introduce fallacies of racial inferiority and promote racial hierarchy. These constructs make the foothold of racism more difficult to dismantle, thereby making participation in a gifted program problematic for gifted African American males (Solomon, Portelli, Daniel, \& Campbell, 2005).

Ogbu's (1992, 2004) theory on voluntary and involuntary groups provides a framework to further understand the racialized attitudes of gifted African America males from the perspective of the historical pathology of immigrant groups. Ogbu's theory makes distinctions between immigrants who have voluntarily chosen to come to the United States in search of attaining the benefits and liberties offered in the Unites States as opposed to involuntary minorities extricated from their native lands against their will and forced into slavery upon arrival in the United States (Ford, Grantham, \& Whiting, 2008). Ogbu concluded that involuntary minorities develop an attitude of what he described as secondary resistance in which they reject the values and beliefs of White culture or other facets of culture perceived to be the cause of their oppression. Applied to the experiences of gifted African American males, it is plausible that current discriminatory practices in gifted programs continue to sour the attitudes of gifted African American males toward participating in gifted programs. The posture of cultural 
incompetency in gifted programs promotes a deficit-thinking paradigm and serves as an exclusionary tactic to hamper the participation of gifted African American males.

\section{Purpose of the Study}

Ford and Whiting (2010) suggested that research examining placement processes as a precursor to acceptance into a gifted program is a vital first step in identifying gifted African American students, specifically African American males who are less represented in gifted programs. Identification and acceptance into a gifted program produces uneven academic and social outcomes for gifted African American males. Moreover, once participating in a gifted program, educational experiences of gifted African American males may generate dissonance between their academic and social self-concept (Scott, 2012). Additionally, incongruence between social and academic outcomes for gifted African American males operates in opposition to research findings that support the positive correlation between self-efficacy and academic performance (Choi, 2005). Considering the influence of self-efficacy on academic outcomes "little research has explored the buffering role of assets (e.g., racial pride, self-efficacy, and self-acceptance) on the relationship between school-based racial discrimination [commonly encountered in gifted programs] and the academic persistence of African American adolescents” (Butler-Barnes, Chavous, Hurd, \& Varner, 2013, p. 1443).

The purpose of this study is to explore the lived experiences of gifted African American males and examine how their sense of self-efficacy shapes the manner in which they counteract or capitalize on the factors influencing their academic persistence. The current study will also contribute to the body of knowledge on the experiences of 
gifted African American males in Grades 6-8, because the majority of studies on AfricanAmerican students' participation in a gifted program and their academic persistence have focused on college-age students (Moore, Ford, \& Milner, 2005). Attention to these grade levels in particular will also expand upon prior knowledge and analyses of gifted African American males and provide a platform for their voices to be heard from their experiences as a middle school student in a gifted program.

\section{Research Questions}

This study focused on the experiences of gifted African American males. The following research questions guided the study:

1. How do gifted African American males understand and experience participation in a gifted program?

2. How do African American males perceive their participation in a gifted program as shaping their sense of self- efficacy and academic persistence?

\section{Significance of the Study}

Disparities in educational outcomes warrant analysis within the context of culture, societal influences, and individual factors, given that one variable alone cannot account for the multi-layered complexities in explaining the pervasiveness of underachievement among African American students (Ford, 2010; Hébert \& Schreiber, 2010). The perpetual combat to circumvent and overcome the barriers initiated and perpetuated by discriminatory practices, extinguishes the motivation and passion essential for African American students to capitalize on a quality, deracialized educational experience (Barton 
\& Coley, 2009). African American males, especially those identified as gifted, are particularly vulnerable to the discriminatory practices associated with gifted program identification and retention protocols.

The underrepresentation of gifted African American males in gifted programs is primarily symptomatic of bias identification measures such as rating scales, informal teacher recommendations, and cognitive ability assessments (Donovan \& Cross, 2002). The lack of attention to the non-academic factors that have damaging influences on their socio-emotional and psychological well-being further sustains the underrepresentation of African American males in gifted programs (Cross \& Vandiver, 2001). More specifically, low teacher expectations, racism, deficit thinking, racial identity issues, and culturally unresponsive curriculum and pedagogy continue to lower rates of African American male participation in gifted programs (Ford \& Moore, 2013). This study is significant in addressing how gifted African American males understand their experiences in a gifted program and how their sense of self-efficacy takes shape while moderating the barriers that show potential for interrupting their academic persistence.

\section{Delimitations}

This study utilizes the qualitative research method of phenomenology. Phenomenological studies provide a means through which the researcher is able "to understand an experience from the participants' point of view” (Leedy \& Ormond, 2001, p. 157). Phenomenology focuses on the manner in which participants perceive and interact with their environment and they ways in which they interpret and make meaning 
from events and situations. Phenomenological studies examine participant experiences and attempts to answer questions associated with the phenomenon of the experience (Creswell, 1998; Williams, 2007). Although guidelines for sample sizes appropriate for phenomenological studies range from five to 25 participants (Creswell, 1998; Morse, 1994), this study draws upon the experiences of 11 participants, representing all of the African American males at the selected site identified as gifted. The number of participants is limited because there is only one school in the school district selected for this study with a sanctioned magnet program for gifted students. Also, the small number of participants may raise concerns with reliability and validity of the study. Conversely, the small sample size encourages meticulous exploration of participant experiences and may increase the depth and breadth of the data analysis.

One of the inherent limitations of phenomenology, is that oftentimes the researcher has associations with participants, or is in some way vested in the population being studied (Williams, 2007). I am an administrator in the school where the students attend. Although I do not have different relationships with gifted students than nongifted students, my position of authority, prior knowledge of the historical practice of identifying gifted students for the magnet, and relationships within the school community, could influence my interpretations of participant experiences. However, my commitment to preserving the integrity of the study allowed me to suspend judgement in reaction to participant responses, without denying my interpretive role as researcher. A discussion of my process for exploring researcher positionality is in the methods section of this study. 


\section{Definition of Terms}

I use the following terms in the context of this study:

- African American or Black- Refers to a person having origins in any of the Black racial groups of Africa (U.S. Census Bureau, May 2010).

- Blackness- Demonstrated by being vocal in expressing one’s opinion, particularly in public settings, having strong work ethic, and actively seeking opportunities to be a leader within the community (Graham \& Anderson, 2008).

- Critical Race Theory (CRT) - challenges the normalization of racism as an acceptable feature in society and reinforces the need to understand the social, economic and historical implications of racism for people of color (Gillborn, 2015).

- Deficit thinking- The belief that culture, beliefs, values, language, practices, customs, and traditions are substandard, abnormal, and unacceptable. Deficit thinking is also grounded in the belief that culturally different students are genetically and culturally inferior to White students (Ford, 2010).

- Gifted- Students, children, or youth who give evidence of high achievement capability in areas such as intellectual, creative, artistic, or leadership capacity, or in specific academic fields who need services and activities not ordinarily provided by the school in order to develop those capabilities (NCLB, 2002).

- Marginalized- Acute and persistent disadvantage in education as distinct from the overall distribution of education opportunities (Education for All Global Monitoring Report, 2009); the "inequitable distribution of resources and 
opportunities” (Ford, 2014, p. 143); the unequal social standing of racial minorities as compared to White inhabitants” (Riphagen, 2008).

- Persistence- personal and cultural assets that causes one to thrive in the face of discriminatory practices (Butler-Barnes, Chavous, Hurd, \& Varner, 2013).

- Phenomenology- a design of inquiry coming from philosophy and psychology in which the researcher describes the lived experiences of individuals about a phenomenon as described by the participants (Giorgi, 2009; Moustkas, 1994).

- Resilience- Inner strength, competence, optimism, flexibility, and the ability to cope effectively in adversity, minimizing the impact of risk factors, such as stressful life events, and enhancing the protective factors, such as optimism, social support, and active coping, that increase people's ability to deal with life's challenges (Abiola \& Udofia, 2011).

- Self-efficacy-An individual's belief and confidence in his or her ability to attain a specific outcome or succeed in a task or pursuit (Bandura, 1986; Bandura, Barbarnelli, Caprara, \& Pastorelli, 2001).

- Underrepresentation-Insufficient or inadequate representation (Oxford Dictionary, 1998).

- Whiteness- American culture that legitimize a station of perceived superiority among a particular group to use property rights as a license to dominate, subordinate, and control others (DeCuir-Gunby, 2006; Harris, 1993; LadsonBillings \& Tate, 2006; Rothman, 1989) 


\section{Organization of Chapters}

The organization of this qualitative phenomenological study is as follows:

Chapter I includes the background of the academic history, barriers, and forecast of gifted African American males in U.S. schools, statement of the problem, theoretical perspectives, purpose of the study, research questions, significance of the study, limitations of the study, and definition of terms. Chapter II provides a review of some of the principal literature used to expound upon and give clarity to the history, benefits, and trends in gifted education. Chapter II also evaluates scholarly studies that examine the barriers to enrollment and participation encountered by gifted African American students as a whole, with a specific focus on gifted African American males. In addition, Chapter II provides an overview of the interdependent relationship between self-efficacy and academic achievement and the critical role it places on the social and academic development of gifted African American males. Lastly, Chapter II discusses CRT, Group Theory, and Deficit Thinking Theory as the theoretical frameworks through which I situate my study and concludes with a summary of the literature findings. Chapter III describes the methodological approach and data collection procedures. Chapter IV presents a detailed analysis of the data and Chapter IV provides a summary of the findings and implications for policy development and future research. 


\section{CHAPTER II}

\section{REVIEW OF THE LITERATURE}

Grim statistics of disproportionality in suspension rates, overrepresentation in special education programs, persistently low test scores, and underachievement in the classroom describe the educational condition of African American males (Graham \& Anderson, 2008). While many of these issues have been in the national spotlight, the calamity in gifted education for African American males has received far less attention. Whiting (2009a) asserted that the existing body of research on Black males offers useful implications for addressing both the needs and issues facing this marginalized group. Yet, scholars have not adequately examined the coalescence of being Black and gifted and male. This chapter explores: a) the historical background and trends of enrollment in gifted programs in the United States; b) benefits of gifted programs c) the barriers to African American's participation in gifted programs; d) the ways in which self-efficacy influences academic achievement and e) the theoretical frameworks through which the experiences of gifted African American males participating in a gifted program are interpreted.

Significant racial disparities, particularly among African Americans are characteristic of the demographic composition of gifted programs across the United States. The body of knowledge concerning gifted African American males reveals a host 
of barriers that they must confront. The existence of these barriers may account for the reason that although African American students account for $16.7 \%$ of the student population, they represent only $9.8 \%$ of students participating in a gifted program. Black males are even more underrepresented in gifted education than all student groups (Ford \& Whiting, 2010; Grissom \& Redding, 2016; US Department of Education, 2010). Henfield (2013) pointed out that it is important to have "an understanding of the interactions between race and gender juxtaposed with giftedness and high achievement that impact the social, academic, and vocational attitudes, behaviors, and subsequent outcomes of this special group of students” (p. 398). That is to say that the convergence of race, gender, and being gifted, cannot be underestimated as predictors influencing how African American males’ approach and persist academically and the extent to which these factors forecast their success in future pursuits. Ford and Moore (2013) emphasized that among the barriers faced by gifted African American males, social factors have high significance. The manner in which gifted African Americans manage issues of racism, prejudice, perceptions of self, and understanding teachers' and peers' perceptions of them in gifted programs are consequential to academic outcomes.

Among the social factors influencing academic outcomes is the significance of self-efficacy. Self-efficacy is an individual's confidence in their ability to engage and be successful is specific academic tasks (Bandura, 1993; Kerpelman, Eryigit, \& Stephens, 2008; Pajares \& Miller, 1994; Uwah, McMahon, \& Furlow, 2008). Choi (2005) found a positive association between academic performance and high levels of academic selfefficacy. Additionally, studies have shown that academic self-efficacy is an indicator of a student's ability to be successful in an educational setting and that students with high 
self-efficacy are academically persistent (Bandura, Barabaranelli, Caprara, \& Pastorelli, 2001; Kerpelman, Erigit, Stephens, 2007; Uwah, McMahon, \& Furlow, 2008). This prior research suggests that African American males' sense of self informs how they construct their sense of self-efficacy and the how they respond academically while participating in a gifted program. To mitigate the impact of these barriers on the academic performance of gifted African American males, policies that drive gifted education should be reflective of the practices that support and sustain the participation of gifted African American males in gifted programs. Furthermore, Ford, Grantham, and Whiting (2008) challenged educators to more effectively recruit and retain minority students in gifted education programs by averting deficit thinking, restructuring identification protocols, and evaluating and responding to their affective and psychological needs. Henfield, Moore, and Wood (2008) support the need for additional research, asserting that "current research literature in the field of gifted education is sparse with regard to the experiences of gifted African American students” (p. 444). This study addresses this deficit in the research literature and explores the following research questions: First, how do gifted African American males understand and experience participation in a gifted program? Second, how do African American males perceive their participation in a gifted program as shaping their sense of self- efficacy and academic persistence?

\section{History of Gifted Programs in the United States}

The inception of gifted education in the United States began in the middle of the nineteenth century with the creation of classes for high-ability students in the St. Louis, Missouri public school system (Piirto, 1999). Piirto documented that similar efforts were 
undertaken as early as 1918 in the Louisville Public Schools in Kentucky. Students with Intelligence Quotients (IQ) scores ranging from 120 to 180 served as the criteria for grouping and services in the classrooms. By the early twentieth century, gifted programs existed in schools across the country (Bhatt, 2011; National Association for Gifted Children, 2008). Prompted by the launch of Sputnik in 1957 and growing concerns of inadequate and uncompetitive education systems in the United States (particularly in science, math, and foreign languages), the National Defense Education Act 1958 (NDEA) was enacted to reform education in the areas of mathematics, science, technology, and engineering. Its goal was to cultivate the brightest minds and position the United States to compete against the scholars of the Soviet school system. NDEA recognized gifted students as an "underdeveloped resource" who with a quality education could benefit American society through their contributions to scientific and technological advances (Jolly, 2009).

Prior to NDEA's acknowledgement of America's underdeveloped resources, the civil rights movement had already engaged in its pursuit of equity for another underdeveloped resource by calling attention to the need to recognize and serve underrepresented populations in gifted education. The watershed ruling in Brown v. Board of Education (1954) Topeka, Kansas affirmed that separate educational facilities were inherently unequal and unconstitutional. This adjudication endeavored not only to desegregate schools, but also to reframe existing paradigms regarding the notion of separate but equal and address subsequent issues related to equitable access to educational programming (Dooley \& Dooley, 2002). Additionally, the Brown decision 
offered the assurance of the attainability of the American ideal of equity and access to educational facilities and programming for people of color.

Despite the decisiveness of the Brown ruling, its implementation met with resistance, which further delayed progress toward changing the educational trajectory for minorities (Gantz, 2004). Progress in education was not only stunted in executing the Brown mandates, but progress in the nation's quest to achieve academic excellence was also challenged by the National Commission on Excellence in Education in the A Nation at Risk: The Imperative for Educational Reform report (National Commission on Excellence in Education, 1983). The report argued that the United States failed to capitalize on gains made in education after NDEA and disputed the adequacy of curriculum, teacher accountability, and student expectations in contrast to more firmly established requirements in other countries for all students to take advanced level courses in mathematics and science (Henfield, Owens, \& Moore, 2008). Although the report received criticism for its depiction of the crisis in U.S. education, the contention sparked a refocusing on areas of much needed reform in the educational system (McIntush, 2000).

Calls for further reforms to gifted education resulted in Congress passing the Jacob J. Javits Gifted and Talented Education Act (1988). Ford's (2014) summary of the central objectives of the legislation indicated that funding was needed to support school districts’ efforts to develop programming for all gifted students. One of the primary objectives of the grant prioritized funding to target the recruitment and retention of underrepresented groups in gifted education (e.g., culturally and ethnically diverse groups, low socio-economic status, students with disabilities and English language learners). In 2002, the Javits Act (1988) was re-sanctioned under the No Child Left 
Behind Act (NCLB). The reauthorization augmented the act to include grants for gifted education services and programs (Bhatt, 2011; Henfield, Owens, \& Moore, 2008). NCLB (2001) required that subgroups (Black, Hispanic, economically disadvantaged, and students with disabilities) meet proficiency standards in order for schools to avoid sanctions. Patrick, Gentry and Owen (2006) asserted however, that NCLB (2001) instilled a mindset based on improvements and diagnosing deficits rather than building upon student strengths. Indeed, the law notably omitted the gifted as an accountability subgroup. They contended that the focus of NCLB (2001) contradicted the empirical studies on motivation theory, which suggests that students learn best when they are empowered to govern their own learning with confidence and enthusiasm.

The rigid mandates of NCLB, its lofty achievement benchmarks, and underfunded nature led to calls for overhauling the legislation. In 2015 Congress reauthorized, the Elementary and Secondary Education Act (ESEA) and President Obama signed the Every Student Succeeds Act (ESSA) into law (ESSA, 2015). The legislation required that a) state report cards include disaggregated data for all students at all levels of academic achievement; b) local education agencies assist schools in identifying and serving gifted students; c) through Title II, which is designated for educator professional development, teachers and principals receive training in identifying and providing instruction for gifted and talented students; and d) through Title IV, which is designated for academic enrichment, high-ability learners are supported through enrichment services funded through the Javits Gifted and Talented Students Education Act (ESSA, 2015). It is too soon to gauge the impact of ESSA on gifted education, however the explicit 
language of the act provides assurances for identification of the gifted, gifted services, educator quality, and funding that was previously non-existent in previous legislation.

The historical landscape of gifted education has experienced continuous change since the mid-nineteenth century, yet revisions to current legislation imply progress has been intermittent, limited in scope, and primarily beneficial to majority populations. Consistent with the historic instability of gifted education, researchers have found that generally, United States classrooms still only scarcely meet the needs of all gifted learners (Archambault, Westberg, Brown, Hallmark, Emmons, \& Zhang, 1993; Moon, Tomlinson, \& Callahan, 1995; Reis, Gubbins, Briggs, Schreiber, Richards, \& Jacobs, 2004). The accountability measures of ESSA (2015), would finally result in improved gifted services that lead to desired outcomes for gifted students including underrepresented populations. Additionally, ESSA (2015) may inspire local education agencies to examine intentionally the manner in which culturally diverse populations access gifted education, thereby decreasing the nation's under-developed resources and pushing gifted education beyond historic limitations.

\section{Benefits of Gifted Programs in the United States}

Gifted programs in the United States (U.S.) offer beneficial curriculum and program models intended to meet the distinct needs of precocious learners (VanTasselBaska \& Brown, 2007). Regular classroom teachers utilize differentiation to accommodate gifted learners in an academically heterogeneous classroom setting (VanTassel-Baska \& Brown, 2007). However, differentiation alone, as an instructional strategy in the regular classroom has been insufficient in advancing the academic achievement of gifted leaners, making gifted programs a more desirable option 
(Hertberg-Davis, 2009). The benefits of gifted programs are linked to the proper execution of delivery models most commonly used in gifted education (e.g., differentiation, acceleration, compacting, enrichment, and gifted resource classrooms) and the competency of the teachers providing instruction to gifted students inside and outside of a gifted program (Rogers, 2007).

Along the same lines, Tomlinson (2003) described differentiated instruction as the teacher's ability to identify the diverse learning needs of students and to create learning experiences commensurate with learning capabilities and interests. Based on this description, differentiated instruction would provide adequately customized instruction for all ability levels in a classroom including those who are gifted. Hertberg-Davis (2009) argued that the model of traditional schooling and teachers' limited time and skill to make the necessary instructional adaptations hinders the effectiveness and impact of differentiated instruction in the regular classroom for gifted learners, substantiating the need for gifted programs. Scholars attributed the diminished capacity of teachers to provide differentiated lessons for students in part to the stresses associated with the highstakes accountability of NCLB (2001). Moon, Brighten and Callahan (2003) asserted that the emphasis on test preparation and remediation for lower achieving students shifted curricular and instructional attention away from gifted students in the regular classroom setting.

Furthermore, Westberg and Daoust (2004) found that even when differentiation occurs in the regular classroom where gifted students are present, teachers focus their attention on struggling students because they assume gifted students are capable of learning with minimal instruction. Research on the effectiveness of teachers with gifted 
education training suggests that preparatory coursework in gifted education affects competencies in selecting and designing curriculum and executing pedagogical practices that meet the needs of gifted learners in a regular classroom (Robinson, 2008). The biennial State of the States in Gifted Education (2015) also documents the lack of readiness of regular classroom teachers, revealing that only 3 out of 38 reporting states confirmed that general education teachers were required to obtain additional training in gifted education beyond their initial certification. This further exposes the need for minimum educator requirements for those teaching gifted populations. It also supports the imperative for gifted programs in which teachers are qualified to provide high-quality instruction for gifted students. Instructional strategies used specifically with gifted students are evidence of high-quality instruction.

For instance, Brulles, Saunders, and Cohen (2010) conducted comparative action research in the form of a quantitative case study. The study examined the clustered grouping practices of gifted students in an urban elementary school. Clustering is a form of student ability grouping based on similar cognitive abilities (Neihart, 2007). Although the school district mandated clustering for gifted students, the researchers determined that some schools had chosen not to follow the model; therefore, the two emergent groups were schools where gifted students were clustered and schools where gifted students were not clustered (Brulles, Saunders, \& Cohen, 2010). Teachers in the schools who did not implement clustering received less professional development on differentiation than teachers in the schools where clustering was utilized. Brulles, Saunders, and Cohen (2010) found that $72 \%$ of gifted students received gifted services in the clustered groups, while only $28 \%$ of gifted students received gifted services in the regular classroom where 
students were not in clustered groups. Analysis of math scores by subgroup (gender, ethnicity, grade, limited English proficiency [LEP] status) revealed that gifted students in the gifted clustered classrooms with teachers who received more professional development experienced greater growth in mathematics than the students who were not in clustered groups with teachers who received limited professional development in gifted education (Brulles, Saunders, \& Cohen, 2010). More profoundly, gifted students in the gifted clustered classrooms demonstrated statistically significant growth in mathematics regardless of subgroup.

The influence of ability grouping on academic achievement among gifted students also has implications for social and emotional development. Researchers have debated the socio-emotional ramifications of ability grouping on gifted students (Calengelo, Assouline, \& Gross, 2004; Neihart, 2007), citing negative impact due to increased pressure to perform academically, feelings of isolation, and distorted self-concept (Hertzog, 2003; Seaton, Marsh, \& Craven, 2009). Subotnik, Olszewski-Kubilius, and Worrell (2012) asserted that in order to elevate the abilities of gifted learners to the level of exceptional, psychological, and social strength is crucial to their overall academic performance. Similarly, Driscoll (2005) affirmed the relationship between socioemotional development and academic outcomes.

To take a case in point, Preckl, Götz, and Frenzel (2010) explored the effects of full-time ability grouping on student's self-evaluations and their experiences with the academic emotion of boredom within a gifted mathematics class. Preckl, Götz, and Frenzel rationalized their study, stating that, "preventing boredom and providing gifted students with appropriate academic challenge are frequently mentioned reasons to justify 
the ability grouping of gifted students into special classrooms” (p. 452). Yet empirical evidence of the influence of boredom on the socio-emotional well-being of gifted students is sparse (Plucker, Robinson, Geenspon, Feldhusen, McCoach, \& Subtnik, 2004; Rogers, 2007). Preckl, Götz, and Frenzel (2010) revealed that there were no differences in measures of boredom between gifted students compared to non-gifted students. Gifted students however, attributed their boredom to a lack of challenging instruction, while non-gifted students attributed their boredom to their teachers over challenging them. Preckl, Götz, and Frenzel claimed that the findings of the underchallenged gifted students' boredom could be understood as a positive effect, since their experience of being underchallenged decreased when they transitioned from the regular classroom to a gifted classroom. The findings of Preckl, Götz and Frenzel are important in affirming that academic challenge is one of the ways gifted students evaluate the quality of their participation in a gifted program (Gentry \& Springer, 2002). Although the findings also indicated that ability grouping for gifted students had a positive influence on their perceptions of increased academic challenge, their self-concept was negatively affected over time. Research is inconsistent in diagnosing the reasons for decreased self-concept of gifted students in a gifted program. However, it may be that changes in self-concept result from what Trautwein, Lidtke, Marsh, and Nagy (2008) referred to as basking-inreflected-glory effect; a feeling of satisfaction experienced by gifted students when they are first identified for a gifted program that wanes over time. Another possible explanation is the contrast-or-habitation effect, which occurs when there is ongoing interaction with other gifted students in a gifted program making individual academic strengths less prominent in the presence of their gifted peers (Brüll, 2009). 
In sum, students assessed three times during the school year, served as a strength of the study. The data collection approach allowed the researchers to investigate the development of the gifted learners over the course of the school year. A limitation of the study is its focus on self-concept and boredom in mathematics classes only, making the generalizability limited. To improve the study, removing academic subject constraints and utilizing a longitudinal approach could make the research findings more robust.

Vogl and Preckel (2014) conducted a quantitative longitudinal study to investigate full-time ability grouping of gifted students from the beginning of their $5^{\text {th }}$ grade year to the middle of their $6^{\text {th }}$ grade year in associating social self-concept and school related beliefs. Data analysis consisted of a repeated-measures multivariate analysis of covariance. Vogl and Preckel found that while there were no effects on social selfconcept of assertiveness, attending the gifted class had positive effects on the student's self-concept as it related to acceptance. Additionally, students in the gifted classes expressed more interest in school and developed better student-teacher relationships than students in non-gifted classrooms.

In a related study, Lee, Olszweski-Kubilius, and Thomson (2012) examined over 1500 gifted students' perception of their self-concept and interpersonal competence. The researchers defined interpersonal competence as the student's ability to interact with peers of the same age. Lee, Olszweski-Kubilius, and Thomson explored how variables related to social competence (age, gender, academic acceleration and participation either in or out of a gifted program) predict social competence. The gifted students perceived their social competence positively and reported high academic and global self-concept significantly higher than social self-concept. Gifted students perceived themselves as less 
socially competent, but having high academic self-concept compensated for low selfconcept which resulted in participants reporting increased self-image overall (Lee, Olszweski-Kubilius, \& Thomson, 2012). Another finding of the study involved differences in interpersonal ability based on the student's experience with subject acceleration. Gifted students who reported higher interpersonal ability had experienced subject acceleration at some point during their educational career. It was unclear however, whether the higher interpersonal abilities made the students ideal for subject acceleration or if subject acceleration contributed to higher interpersonal abilities.

Specifically, acceleration is a strategy used to meet the learning needs of gifted learners that involves moving students through academic content more rapidly than standard curriculum pacing and beyond typical age chronology of schooling timelines (Colangelo, Assouline \& Gross, 2004). Wells, Lohman, and Marron (2009) purported that accelerated students have the ability to keep up with and even exceed the academic performance of older peers. Studies have also shown that acceleration contributes to positive academic achievement results as well as positive attitudes toward school and future aspirations (Noble, Robinson, \& Gunderson, 1993; Sethna, Wickstrum, Boothe, \& Stanley, 2001). Amidst reports of the benefits of acceleration, concerns regarding the impact of grade-based acceleration on socio-emotional preparedness have contributed to the underutilization of this strategy (National Association for Gifted Children [NACG], 2009; Southern \& Jones, 2004). In another study, Seigle, Wilson, and Little (2013) quantitatively explored teacher and administrator $(\mathrm{n}=152)$ perceptions regarding acceleration as a viable strategy for gifted learners. The sample consisted of educators in these role groups from suburban, urban, and rural school districts. Survey analysis 
indicated that teachers in this sample supported acceleration, but they perceived administrators and parents to be less receptive to acceleration because of the social implications of mixed age groups. Current research does not support the notion of harmful socio-emotional effects of acceleration and research on the social benefit of acceleration is minimal (Seigle, Wilson, \& Little, 2013).

The studies of instructional delivery models as it relates to the academic and socio-emotional development of gifted learners provides clarity in understanding the benefits of participating in a gifted program. Based on the research referenced in this section, curriculum challenge levels and the extent of educators' expertise exhibited in teaching the gifted mediate the benefits of gifted programs. Transcending their academic achievement, gifted learners contended with normalizing their academic prowess with social factors that influenced their perceptions of self and how they interacted with teachers and peers. Attention to the instructional nuances within gifted programs invites an open-minded approach to the ways in which gifted programs might elevate the learning experiences of gifted students.

\section{Trends in Gifted Enrollment in the United States}

The National Center for Education Statistics (NCES, 2015) reported a 7\% increase in the U.S. student population between 2000 and 2014, reaching 50.3 million students enrolled in U.S. schools in kindergarten through twelfth grades. By the end of 2014, the White student population decreased from 28.3 million to 24.9 million and the African American student population decreased slightly from 8.4 to 7.8 million. The Asian/Pacific Islander student population increased from 2.2 million to 2.6 million and the Hispanic student population (being the second largest minority student group) 
increased dramatically from 9.3 to 12.8 million. Considering the downward trend of growth patterns of White and African American students, the general student population is expected to grow another 3\% by the year 2026 (NCES, 2015). Reviewing the enrollment trends of student populations in U.S. schools provides a baseline for analyzing demographic trends in gifted program participation. It presents implications for devising strategic recruitment and retention practices for gifted programs based on growth patterns of underrepresented ethnic groups.

Despite declines in White student enrollment in gifted programs, representation for White students in gifted programs remains disproportionately high. Comparatively however, Hispanic students comprise $22.3 \%$ of the student population, but constitute only 15.4\% of students receiving gifted services. African American students comprise 16.7\% of the student population, but make up just $9.8 \%$ of students enrolled in gifted programs (U.S. Department of Education, 2010). In fact, these statistics confirm the continued underrepresentation of African American students that has persisted for more than seventy 70 years (Ford, Moore, Milner, 2005).

Moreover, researchers have expressed concern regarding these disproportionalities due to the prospect of inequitable and discriminatory practices inflicted upon minorities (Baker, 2013; Ford, 1998). Concern regarding the enrollment of minority groups in gifted programs stems from the link between enrollment trends and the manner in which school personnel identify minority students for gifted programs. Researchers have found that even when students of color qualify for gifted services, they are less likely to be referred to a gifted program or receive gifted services than their White counterparts (Ford, Grantham \& Whiting, 2008; McBee, 2006). 
In order to be selected for enrollment to a gifted program, it is crucial to define giftedness and how it functions as a precursor in the decision-making process for selection. There are many terms used to describe gifted children such as genius, precocious, prodigy, and talented. Definitions for giftedness are as multifarious as the terms themselves. The seminal work of Sydney Marland, commonly referred to as the Marland Report (1972), offered the federal government's first definition of giftedness. Marland defined giftedness as high performance in academics, creative and productive thinking, leadership and the performing arts and encouraged states to identify 3-5\% of their school population as gifted.

Most school districts use intelligence quotient (IQ) or achievement tests to identify students as being gifted (Davis \& Rimm, 2004). This means little has changed from the early 1900's when IQ tests were the primary instrument used to identify and group gifted students (Piirto, 1999). Although intelligence and achievement tests readily identify middle-class White students, they fail to effectively identify African American, Hispanic/Latino, and American Indian students (Naglieri \& Ford, 2005; Skiba, Knesting, \& Bush, 2002). Consequently, gifted programs are disproportionately overrepresented by the White middle class (Ford, Grantham, Whiting, 2008). Reliance on IQ or achievement testing as a singular measure for giftedness, and its exclusionary impact on underrepresented populations, prompted the need for a more expansive definition (Borland, 2003; Pfieffer, 2003; Tomlinson, 2003, 2009; VanTassel-Baska, Feng, \& Evans, 2007).

The United States Department of Education (USDOE, 1993) broadened the Marland Report’s (1972) definition to recognize “outstanding talent present in children 
and youth from all cultural groups, across all economic strata, and in all areas of human endeavor” (p.19). The specificity of the inclusive language and similar iterations of definitions by individual states have signified positive steps toward inclusion (McClain \& Pfeiffer, 2012). Additionally, with a more expansive definition of giftedness, there are hopeful implications for the identification and recruitment of African American students into gifted programs. Hopeful implications however, do not negate the prevalent barriers of gifted African American students in having comparable experiences in gifted programs as their White counterparts due to their unique social, academic, and cultural needs (Bonner, 2000).

\section{Barriers to African American Enrollment in Gifted Programs}

As shown previously, evidence reveals the underrepresentation of African American students in gifted programs (Moore, Ford, \& Milner, 2005). African American students are underrepresented by $48 \%$, which represents the absence of 253,000 African American students who should be identified as gifted and participating in a gifted program (Ford, Grantham, \& Whiting, 2008). There are hosts of issues that have contributed to and perpetuate the underrepresentation of African American students in gifted programs. Researchers have identified the most pervasive issues to include standardized assessment, referral processes, socioeconomics, peer influences, deficit ideologies, underachievement, racial identity, social injustice, and ineffective policies (Ford, 2010; Henfield, Owens, \& Moore, 2008; Michael-Chadwell, 2010; Moore, Ford, \& Milner, 2005, Pfieffer, 2003; Romanoff, Algozzine \& Nielson, 2009; Whiting, 2009). The host of barriers placing gifted African American students in a position of 
disadvantage compared to their White counterparts, demands an educational shift in both proclamation and practice.

Gifted children from culturally diverse backgrounds are at a disadvantage because of the cultural biases embedded in commonly used standardized measures to identify the gifted (Louis, Subotnik, Breland, \& Lewis, 2000). The urgency for revisions to standard assessment instruments is based on the belief of researchers that the evidence of knowledge and skill are demonstrated in the context of culture and the way in which tasks are approached because of culture (Baldwin, 2004; Brown, Collins, \& Duguid, 1989; Ford, 2006; Resnick et al., 1991). Romanoff, Algozzine, and Nielson (2009) conducted a quantitative study using a post-hoc causal comparative quasi-experiment to compare the achievement of African American students and White students (n=198) over a 4-year period. The two groups of students selected for the study were students identified and placed in a gifted program using the same qualifying assessment criteria as their peers who also met the requirements for placement in the gifted program, but did not participate. The researchers hypothesized that assessment scores in mathematics and reading for students in the gifted program would not be different between the two groups of students. Romanoff, Algozinne, and Nielson also sought to determine if the end-ofgrade mathematics scores between the African American and White students identified for participating in a gifted program would be different from their peers who qualified for a gifted program, but did not participate.

The findings of the study indicated that students selected for the gifted program and participating in the gifted program scored significantly higher on the end-of-grade tests in reading and math than the students who were equally qualified to be in the gifted 
program, but did not participate. Consistent with perpetual achievement gaps between African American and White students (Donovan \& Cross, 2002), African American students in the gifted group scored below that of their White peers in the gifted group. However, the differences in mathematics and reading were smaller than the achievement gaps that existed between African American and White students identified as gifted, but did not participate in a gifted program. Notwithstanding the gap between African American students and White students, the findings deliver hopeful indicators to the academic advantages to gifted African American students participating in a gifted program considering that the scores of the African American students were far above grade level. Moreover, African American students in the gifted program scored higher in reading and mathematics than academically comparable White and African American students identified for and who did not participate in a gifted program.

A limitation of this study is the utilization of only two assessment measures to compare achievement between the two groups. Romanoff, Algozzine, and Nielson (2009) proposed that schools confronting the issue of disproportionality in a gifted program should use multiple data sources to identify gifted students. They cautioned that educators should not make assumptions about the inability of African Americans participating in a gifted program based on lower standardized assessment scores than their White peers, taking into account that assessment models for the identification of minority populations are inadequate. Romanoff, Algozzine, and Nielson stated that "one way to increase minority participation at the highest level of production and performance is to increase student enrollment in educational programs for the gifted and help them gain the thinking dispositions that lead to improved performance” (p. 170). Romanoff et 
al. suggested that the positive outcomes among African American males in a gifted program may be reduced without adequate academic supports to ensure their success.

Along the same lines, Grissom and Redding (2016) conducted a quantitative study using regression analysis to analyze the relationship between assignment to gifted programs and student-teacher race congruence. The study addressed the extent to which African American and Hispanic elementary school students were assigned to gifted programs at a disproportionately lower rate than White and Asian students. Grissom and Redding also sought to uncover if the disproportionality resulted from other observable student characteristics and to determine if own-teacher race increases the probability of non-White student placement in a gifted program. To contextualize the processes associated with student assignment to gifted programs in relation to own-teacher race behaviors, specifically as it relates to gifted program assignment, the researchers used bureaucratic representation theory. The theory suggests that "teachers of color are more likely than White teachers to exercise discretion on behalf of students from their same racial or ethnic background-and similarly for White teachers and White students-such that students' probabilities for being assigned are higher with own race teachers” (p. 2). In fact, research indicates that African American and Hispanic students have higher rates of participation in gifted programs in schools where there are higher numbers of African American and Hispanic teachers (Nicholson-Crotty, Grissom, \& Nicholson-Crotty, 2011; Rocha \& Hawes, 2009).

The results of Grissom and Redding's (2016) study showed that disproportionalities in race and ethnicity in the identification of gifted students had little to do with academic performance. They found that mathematics and reading scores, 
school location, and the characteristics of individual classrooms, in addition to teacher race, contributed to gifted assignment probabilities. African American students in classrooms with non-African American teachers were routinely less likely to receive gifted services, particularly in reading. Card and Guiliano (2014) asserted that such practices steer high-achieving African American students with non-African American teachers away from gifted services and the benefits found therein. Several researchers detailed how representative bureaucracy theory may explain assignment practices of nonAfrican American teachers. These factors include: (a) the manner in which teachers execute their power as referral gate-keepers (referral, diagnosis, and selection) based on race and ethnicity resulting in the disproportionality; (b) teachers recognize giftedness and potential giftedness in students of races other than their own based on background, biases, and expectations; (c) different student behaviors with an own-race teacher make their gifted characteristics easier to identify; and (d) parents engage with own-race teachers more comfortably leading to involvement with the assignment process itself (Gershenson, Holt, \& Papageorge, 2015; Grissom, Kalogridges, \& Loeb, 2015; Lim, 2006). With regard to predicted probabilities based on observable student characteristics, White students had a $6.2 \%$ predicted probability of assignment to a gifted program compared to a $2.8 \%$ predicted probability of assignment to a gifted program for African American students which represented a statistically significant difference (Grissom \& Redding, 2016). This means that African American students are 3 times less likely to receive gifted services in classrooms with non-African American teachers than with African American teachers. They did not find a significant correlation between gifted 
assignment and race congruence among White, Hispanic, or Asian students (Grissom \& Redding, 2016).

Grissom and Redding's (2016) study is limited in that it does not examine unobserved school characteristics, such as school-based evaluation and referral processes, however the predicted probabilities offer strong evidence for schools to acknowledge and take action to reverse discriminatory and bias assignment practices that lead to underrepresentation in gifted programs. Grissom and Redding (2016) explained that if hindrances to gifted programs for African American students are the result of teacher discretion and common knowledge, where race congruence is an underlying factor, there needs to be expedient revisions to policies and protocols. These revisions should involve drawing upon multiple data sources rather than an individual autonomously making decisions regarding gifted assignments (Card \& Guiliano, 2014). If changes are not made "minority students will continue to be underrepresented in programs for the gifted as long as there are inconsistencies between the definitions of giftedness and the instruments used to identify giftedness” (Romanoff, Algozzine, \& Nielson, 2009, p. 171). Therefore, it is incumbent upon national and local level gatekeepers of gifted programming to revamp and realign criteria and assessment tools to be void of the biases that have perpetuated the underrepresentation of students of color in gifted programs.

For example, Barlow and Dunbar (2010) claimed that attempts to ameliorate identification practices for gifted students have uncovered insinuations of the operation of racial bias, particularly favoring White and middle-class children. Barlow and Dunbar engaged in a 15-year case study of a large Midwestern school district to examine how schools preserved limited and imbalanced access to gifted and talented services through 
practices that secure the advantages and pre-eminence of whiteness. Using Harris' (1993) theoretical framework of property, which asserts that in American culture and its legal system "whiteness" is a form of property that perpetuates inequitable practices that insulate access to gifted programs with partiality to White students. Whiteness as property refers to the laws in American culture that legitimize a station of perceived superiority among a particular group to use property rights as a license to dominate, subordinate, and control others (DeCuir-Gunby, 2006; Harris, 1993; Ladson-Billings \& Tate, 2006; Rothman, 1989)

Harris (1993) argued that the concept of whiteness as a property is evident in the historical ownership of slaves to the expropriation of land and land rights from Native Americans. Attitudes regarding property rights are pervasive in society today and uphold "the settled expectations of relative White privilege as a legitimate and natural baseline” (Barlow \& Dunbar, 2010; Harris, 1993, p. 1714). Barlow and Dunbar (2010) interlaced Harris' (1993) theory with identification practices for gifted students, contending that White property mindsets are ignored as a variable influencing the disproportionate numbers of White students in gifted programs. Harris' theory points out that whiteness as property involves: (a) exclusion rights; (b) maintaining rights of disposition; (c) rights associated with reputation and status from the acquisition of property; and (d) right to sustained usage and pleasure. Barlow and Dunbar maintained that as it relates to exclusion rights, identification systems and practices work against culturally different students and these systems provide white students access to gifted programs without consideration to the exclusion of non-Whites. Maintaining rights of disposition implies that condoning biases in identification systems affirms the right of 
White students to maintain their position in a gifted program without consideration for non-White students who are equally qualified to maintain the same position.

Furthermore, parents of White students utilize status and reputation, commonly based on economic power, thus enforcing racial bias in the identification process. In many instances, minority parents of gifted children have inadequate financial and social capital, which leads to lower rates of identification and enrollment to a gifted program (Mickelson, 2003). Harris (1993) points out that Whites used slaves to work and bring them profits from land that they had stolen from Native Americans. As such, the nature of the disproportionality of White students to non-White students in gifted programs indicates their utilization of gifted services funded by the state. In other words, White students reap the benefits of gifted programs from the contributions of White and nonWhite taxpayers collectively, but non-White gifted students are scarcely the recipients of those same services to which they should have equal access.

The findings of the Barlow and Dunbar (2010) case study revealed that identification processes over a 25-year period supported the inclusion of White middleclass students in a gifted program compared to non-White gifted students of lower socioeconomic status. Selective criteria in the form of a single measure was used to determine selection to a gifted program and access to testing information and guidance for the interpretation of testing data was restricted. Screenings based largely on teacher and parent recommendations limited the same participation by less informed non-White parents. Furthermore, the intricate identification process made it difficult for non-White parents to navigate. The state used out-of-state students to satisfy desegregation 
guidelines. Alarmingly, disproportionality in access to gifted programs has increased commensurate with increasing growth among minority populations.

Hence, inferences drawn from this case denote that race continues to be a factor in the enrollment of underrepresented groups in gifted programs. Lewis (2003) and Rogoff (2003) contended that although education presents an ideal system for the mitigation of negative influences of race and class, discrimination within schools continues to perpetuate inequities because of both. They argued that whiteness is a norm embedded in school culture and is sustained through beliefs and behaviors exhibited in the daily workings of schools. Barlow and Dunbar (2010) suggested that the findings of this study can be used to "guide the appropriate use of power to undo the biases of white property and enable all children to receive the educational opportunities that best serve their interests, talents and needs” (p. 83). Plainly stated, power used responsibly within the context of the educational setting, can eliminate partiality whereby all students can be the beneficiary of services and programs designed to meet their needs.

The giftedness of African American students goes undiagnosed because of the standardization of practices that limit access to gifted programs and perpetuated by the inherent nature of cultural bias in identification protocols. Cultural bias in gifted education is a function of: (a) racial partiality in the exclusionary criteria established by gifted program gatekeepers, (b) constrained assessment instrumentation that ignores the non-traditional characteristics of culturally different gifted students, and (c) the disregard for racially based socio-emotional pressures experienced by African American students identified as gifted or participating in a gifted program. African American students then, are at a significant disadvantage in benefiting from gifted services designed to nurture 
and sustain their passion for learning like their non-minority counterparts. The academic prognosis for gifted African American males is even more austere.

\section{Barriers to African American Male Enrollment in Gifted Programs}

To illustrate this point further, federal and state agency data indicate that there are low percentages of gifted African American males participating in gifted programs. National survey data showed that in U.S. public schools, gifted African American males

and females participating in gifted programs were noticeably underrepresented (Hargrove \& Seay, 2011; NCES, 2006). Trends in the enrollment of gifted African American males informs our understanding of how their underrepresentation in gifted programs are potential predictors of negative future outcomes typically associated with African American males such as low educational attainment leading to bottom-rung employment and nominal wages, poor health, and the increased likelihood of involvement with the criminal justice system (Harvey, 2008). Holzer (2006) contends that gifted African American males matriculate from formal education, but societal disadvantage impedes their progress and their giftedness goes unrecognized. This further substantiates the urgency in addressing the deficits that permeate existing gifted programs.

There is increasing evidence to support the relationship between African American males who underachieve in school and the rate at which they are incarcerated (Howard, 2010). Scholars infer relationships between negative school experiences and outcomes after exiting the school system (Franklin, 2007; Hopkins, 1997; Howard, 2008). African American males represent the largest ethnic group in prisons, with incarceration rates at five times more than White males. Trend data predicts that one in three African American men may be imprisoned, paroled, or probated at some time 
during their lives (U.S. Department of Justice, 2006). These grim projections fuel the need to investigate patterns of enrollment among gifted African American males' future outcomes.

Hargrove and Seay (2011) investigated African American and White teachers' perceptions of non-school related and school related barriers limiting the number of African American males from participating in a gifted program. Questionnaires were given to 370 teachers who were selected using a randomized sampling technique. Data from the questionnaires were used to analyze responses based on race and ethnicity and the teacher's acquisition of gifted education professional development. Frequency counts derived from the questionnaire classified major, moderate, and minor obstacles to African American males participating in a gifted program. Hargrove and Seay identified differences in language experience and lack of a stimulating home environment as the two major barriers limiting participation in a gifted program.

Of the 10 questions addressed by the respondents, none of the questions identified issues involving or associated with teacher behavior as a barrier to African American males participating in a gifted program. For example, prejudicial attitudes held by the teacher or the teacher's inability to recognize potential giftedness were rated as minor barriers. This contrasts researchers' assertions that the low African American male representation in a gifted program is strongly related to the actions and belief of teachers who serve as gatekeepers to gifted education programs (Elhoweris, Mutua, Alsheikh, \& Holloway, 2005; Ford \& Whiting, 2008; Speirs Neumeister, Adams, Pierces, Cassady, \& Dixon, 2007). Hargrove and Seay (2011) found that White teachers identified non-school related barriers limiting participation to a gifted program, whereas, minority teachers 
identified both school related and non-school related barriers limiting participation to a gifted program. White teachers were more likely to agree that giftedness was not valued by the African American community, consequently encouraging African American students to perform poorly. The most profound differences were between White and African American teachers' perceptions for the reasons African American males disengage from school. Consistently, White teachers cited non-school related barriers such as home environment, membership in a cultural group that does not value giftedness, language experiences, and the use of nonstandard English. These results endorse Hyland's (2005) claim that "unwitting racism and weariness, with being blamed for Black student underachievement, explains why White teachers look outside of schools to understand disparity” (p. 97). This implies that White teachers lacking adequate professional development in the area of culturally responsive learning environments, or who themselves lack cultural competencies, misidentify the true source of their inability to connect with, empower, and teach African American males when they remove themselves as a contributor to their lack of success in the classroom.

Henfield, Moore, and Wood (2008) conducted a qualitative study using Critical Race Theory as a theoretical framework to shed light on how gifted African American males perceived their experiences in a gifted program. The researchers utilized a grounded theory approach, drawing upon semi-structured interviews with 12 gifted African American males. Henfield, Moore, and Wood found that peer influences, deficit ideology, gender, acting White, and acting Black shaped the ways in which gifted African American males understood and responded to their academic environment revealing that the social context in which gifted African Americans existed was not easily navigated. 
Gifted African American males contended with complex social dynamics within the gifted program by exhibiting academic disengagement and gifted "identity distancing” (p. 442). Consequently, Henfield, Moore, and Wood called for closer examinations of the lived experiences of African American males in gifted programs. Ford and Whiting (2010) urged that “we must consider quality of life in gifted classrooms-student’s sense of belonging, relationships with peers and educators, academic support, expectations of self and others, and academic identity and self-perceptions” (p. 133). Therefore, it is not acceptable for African American males to simply be represented in gifted programs, but they are deserving of experiencing a non-toxic, nurturing environment in which they can thrive socially and academically.

Whiting (2009a) underscores the gravity of illuminating issues affecting gifted African American male achievement. A student identified as gifted would innately exercise the academic exceptionality associated with giftedness, however Whiting identified a number of achievement barriers facing gifted African American males including identity as self-perception, peer pressures, social injustices, beliefs about achievement, and notions of masculinity. Synthesizing the study of Henfield, Moore, and Woods (2008) with Whiting's (2009) study, the academic barriers encompass areas of intersectionality-where academic outcomes are predicated upon and interwoven with social context. Barriers to academic achievement are played out in what researchers refer to as cool pose (Graham \& Anderson, 2008; Majors \& Billson, 1992; Tatum, 2005; Whiting, 2009). Cool pose is a persona of indifference, aggressiveness, and obstinacy that gifted African American males adopt as a means of preserving dignity and dealing with oppression and pressure from external forces (Whiting, 2009). Some aspects of the 
cool pose persona closely relate to Ford, Grantham, and Whiting's (2008) descriptive and exploratory study of peer pressure among ( $n=66)$ gifted Black students. Ford, Grantham, and Whiting noted that a large number of students attributed their lack of performance in school to overwhelming peer pressure. The researchers also found that many of the students masked their intelligence by exhibiting behaviors associated with "acting Black" such as using slang, talking loudly, acting mean, thuggish or gangsta (p. 230). Based on the findings of this study, subversive behaviors indicative of cool pose and acting Black exhibited by gifted African American males are coping strategies to avoid exclusion from their peer group. As such, using a broad brush to characterize the social disposition of gifted African American males is unfitting.

To expand upon this point, Graham and Anderson (2008) engaged in a microethnographic case study in which the participants distinguished between the negative connotations of "acting Black" or being thuggish and the concept of "Blackness" (p. 474). The study explored the extent to which three gifted African American male high schoolers valued educational attainment, the extent to which they connected with their ethnicity, and the influence of how those they considered to be significant in their lives either encouraged or discouraged the development of their academic and/or ethnic identity. The participants considered Blackness to be a source of empowerment and motivation and exhibited what they believed to be behaviors of Blackness such as "being visible and outspoken in the public eye, having an undying and tireless work ethic, and a desire to serve their communities as servant leaders” (p. 493). The participant voices collectively resounded with the notion that "to them, the real sign of individuality and manhood was to be oneself in the face of social pressure and criticism” (p. 493). 
Additionally, the findings revealed that, "higher achieving students would be more likely to have a positive racial self-esteem that included ‘one’s Blackness’ as positively valued and desired” (Saunders, Davis, Williams \& Williams, 2004, p. 83). When gifted African American males perceive their Blackness as a source of empowerment, they are more likely to possess positive racial self-esteem and higher levels of self-efficacy, which in turn influences favorable academic outcomes. These finding serve as a promising counter-narrative to the negative influences associated with the notion of acting Black.

According to Hsieh, Sullivan, and Guerra (2007) extensive research has examined the relationship between self-efficacy and achievement. They concluded that, in general, confident students are more willing to persist in the face of adversity. Similar research studies also indicate that the interplay between self-perception and self-efficacy influence social and academic outcomes for gifted African American males, concluding that without the appropriate social and academic supports, gifted African American males are in jeopardy of succeeding in a gifted program (Bonner, 2003; Ford, Grantham, \& Whiting, 2008; Henfield, 2013; Wiggan, 2007). Bonner (2003) conducted a phenomenological case study to understand the experiences of two gifted African American male collegians, focusing on the students' relationships with their respective schools. As it relates to self-perception, Bonner found that the gifted African American males negotiated the idea of being intelligent and being a high achieving African American male. Bonner (2003) attributed this self-perceived dichotomy to be what Dubois (1986) referred to as an "unreconciled striving”; "being forced to become two different people in one situational context” (p. 31). This concept alone, undergirds the exigency for further excavation on this topic. 
In fact, the under-examined issues of gifted African American males associated with identification, recruitment, and the social and academic context in which they learn and are socialized in a gifted program presents an opportunity for research to be extended. Ford, Moore, and Scott (2011) asserted that we must be "assertive and proactive for the current and future African Americans who are gifted but for whom traditional practices, culturally unresponsive practices and attitudes, beliefs, policies, practices and instruments, have proven to be unhelpful or even harmful” (p. 251). This is particularly important because traditionally African American males have affirmed their racial identity, sense of pride, self-efficacy, and self-esteem by excelling in sports and the arts (Hébert, 2002; Sailes, 2003). African American males often distance themselves from the notion of being both Black and academically successful, therefore it is imperative to transform the manner in which they envision themselves in an academic environment (Whiting, 2009a). Based upon the research on gifted African American males’ self-perceptions, Callahan (2005) suggested that even if students are identified successfully and participate in a gifted program, participation ultimately is not advantageous if improved quality of life and self-satisfaction are not cultivated. Bandura, Barbarnelli, Caprara, and Pastorelli (2001) found that when students have higher levels of self-efficacy they are more likely to persist, which captures the need to investigate the specific role self-efficacy plays in gifted African American males' persistence in a gifted program through the lens of their lived experiences. 


\section{Self-Efficacy and Academic Achievement for African American Males in Gifted \\ Programs}

There is a strong association between self-efficacy and academic goals and performance among African American students (Kerpelman, Eryigit, \& Stephens, 2008). Researchers found that sense of belonging, academic self-efficacy, and educational aspirations are positively correlated with academic achievement among minority groups which alludes to its merit for gifted African American males (Eccles, Wingfield, \& Schiefele, 1998; Rojewski \& Kim; 2003). Quality of life in a gifted program, which encompasses sense of belonging, relationships with peers and teachers, academic safety nets, expectations, academic identity and self-perceptions, are essential to the success of gifted African American males (Harmon, 2002; Louie, 2005). Racial identity of African American males is constructed from the belief that their identity is realized in personal pride, abilities, and sense of self-efficacy (Phinney, 1990). Bandura’s (1977) seminal work on self-efficacy, helps to establish the context for negotiating the idea of selfefficacy intertwined with academic achievement. For the purpose of this study, selfefficacy refers to a student's need to achieve, and their willingness to make the needed sacrifices to achieve. Research indicates that even when contending with negative stereotypes and the uncomfortable social dynamics that exist for them in a gifted program, African American males who have an elevated sense of self-efficacy are able to persist academically. They persist academically by disavowing notions of their incapability and believe in their intellectual and creative giftedness (Whiting, 2006). While self-efficacy is an academic characteristic among highly motivated and high achieving students, it holds exceptional significance for African American males 
because of its relationship to their racial identity and self-concept (Platt, 2002; Sailes, 2003). Researchers suggest that African Americans confront more barriers to racial identity development than White students (Helms, 1985; Parham, 1989; Smith, 1989; Spencer \& Markstrom-Adams, 1990). Gifted African American males will excel academically if they have a well-developed racial identity and healthy self-concept. Conversely, "Black males who have an underdeveloped sense of academic identity are less likely to persist in school” (Whiting, 2006, p. 223). The success of African American males participating in gifted programs hinges on their scholar identity, meaning their identity as a student, which is central to self-efficacy. Ford (1996) and Hilliard (2003) determined that high resilience, self-confidence, self-control, selfresponsibility, and clarity about tasks to be completed and their competence in completing tasks thoroughly exemplifies a strong sense of self-efficacy for gifted African American males.

To further expand the connection between self-efficacy and academic outcomes, Uwah, McMahon, and Furlow (2008) examined the relationships between perceptions of school belonging, educational aspirations, and academic self-efficacy among African American male $(\mathrm{n}=40)$ high school students. The participants completed a sense of belonging inventory and responded to statements such as: (a) people at this school are friendly to me, (b) people here notice when I'm good at something, and (c) I feel like a real part of this school. They also completed a self-efficacy index requiring them to respond to statements about their academic abilities using a Likert scale ranging from $1=$ far below to $5=$ very much above. Results from the study indicated that students felt a strong sense of belonging to the school when participation was intentionally solicited, 
however students’ perceptions about being liked did not influence their academic selfefficacy. Research supports that academic self-efficacy is more significant to academic achievement than self-concept and self-esteem (Jonson-Reid et al., 2005). McCleland's (1961) need to achieve theory supports that African American males who possess scholar identity have a stronger need to perform academically than to be affiliated with individuals or groups. This may account for the reason that it is more important for African American males to be acknowledged for what they are able to do, than for being liked (Uwah, McMahon, \& Furlow, 2008.) This belief can also be reasonably applied to African American males enrolled in gifted programs (Thomas, 2009).

The use of appropriate measurement scales to determine the relationships between self-efficacy and sense of belonging are strengths of Uwah, McMahon, and Furlow's (2008) study. However, the study is limited in drawing conclusions about the influence of self-efficacy on academic achievement because academic outcome data was not considered. Strengthening this study would involve using a larger sample size and incorporating specific academic data to find correlations between academic performance and self-efficacy. Although this study did not find a direct connection between selfefficacy and academic outcomes, previous studies have linked self-efficacy to academic performance (Choi, 2005).

For example, Noble (2011) investigated the impact of self-efficacy beliefs of African American male collegians in relation to their motivation to excel in mathematics. He used Bandura’s (1986) social cognitive theory as the theoretical underpinnings in understanding self-efficacy and its influence on driving change in one's self and their circumstance. Social cognitive theory delineates four indicators of self-efficacy 
(Bandura, 1986). Firstly, enactive attainments refer to the manner in which experiences impact efficacy, in which case, past successes or failures with specific endeavors will either raise or lower one's sense of self-efficacy. Second, vicarious experience is the belief in one's ability to complete a task successfully after observing someone of similar disposition complete the same task. Verbal persuasion is one's ability to articulate and convince others of their ability to complete a task. Lastly, physiological state are physical behaviors that infers one’s ability to complete a task. Pajares (2002) concluded that an individual's level of self-efficacy influences the type of task they attempt to achieve.

Using a culturally appropriate qualitative research method, Noble (2011) addressed how African American males’ self-efficacy affected choosing tasks, persistence, and exerting effort in a collegiate mathematics class. From the data analysis of interviews and mathematics autobiographies, Noble found that self-efficacy, in terms of vicarious experience, and enactive attainment, had the most influence on academic outcomes in mathematics. Based on the results of the study, Noble stated that there is an imperative for "an aggressive effort to immediately provide more models of success to African American male youth so that they will have accessible mechanisms to help counter the many perceptions that exist with regard to their academic achievement capabilities” (p. 203). As the findings relate to gifted African American males, it is appropriate to draw upon Whiting's (2006) conceptual mode of the scholar identity reinforcing an intentional focus on gifted programs in developing a scholar identity. In so doing, more African American males may feel a sense of belonging [in the program], 
will find value in the educational experience, and capitalize on the benefits inherent to participating in a gifted program.

\section{Critical Race Theory and its Application to African American Male Enrollment in Gifted Programs}

Based on the context and purpose of this study, Critical Race Theory (CRT) serves as the conceptual underpinning to contextualize and give clarity to the impetus for this study. CRT had its beginnings in the 1970's in response to the manner in which the critical legal studies movement inadequately engaged in confronting the impact of race and racism embedded in U.S. laws and policies (DeCuir \& Dixson, 2004). CRT formed in response to the critical legal studies movement by acknowledging race in the formation of inequitable practices and addressing these inequities through social justice as a means of promoting positive socio-cultural change for marginalized groups (DeCuir \& Dixson, 2004). CRT scholars Derrick Bell, Mari Matsuda, Richard Delgado, and Angela Harris challenged the improprieties of the law and its implications for people of color (Tate, 1997). Their opposition to the existence of societal inequities based on race gave shape to the basis of CRT. Critical Race Theory holds that (a) racism is an inherent feature of society in daily life and that the ideologies of racism are so deeply embedded in the political and legal constructs of society, they are interpreted as being normal behaviors and unrecognizable; (b) CRT opposes the notion that standards of society are based solely on the experiences of White Americans, but validates the value of the experiences of people of color in their fight against oppression through "the use of literary narrative knowledge and storytelling to challenge the existing social construction of race;” and (c) 
challenges the liberal belief that the law as written provides equity and justice for all people in society (Parker, 2008, p.2). Therefore, the telling and re-telling of these personal accounts legitimizes the realities of the influence of race and confronts the discriminatory practices that have camouflaged themselves through the normalization of racism.

Based on the pervasiveness of the issues of race and racism as described in Critical Race Theory across all facets of society, the field of education is not exempt from the negative and perpetual impact of racially based inequities and injustice. Parker (2008) succinctly encapsulated the essence of CRT as it relates to education when he stated:

Critical race theorists seek to break the dominance of storytelling about the success of merit, equality, the market, and objectivity that are so deeply entrenched and accepted unquestioningly by larger society, through the legitimizing of legal narratives of racial discrimination and the power of the law used against persons of color. The importance of the narrative for purpose of social justice, and the implications of the intersections and conjunctions related to difference, are just a few of the various aspects of critical race theory that have important implications for education. (p. 194)

To better understand these implications for education, Ladson-Billings and Tate (1995) introduced CRT to education as a lens through which to examine the collusion of race, culture, and equity in education practices, policies, and research. Given the literature on the achievement of African Americans in relation to the achievement of their White counterparts, Ladson-Billings and Tate called for more attention to the interplay of race 
and equity among African Americans. More specifically, attention should be given to subgroups within African American student populations, such as those identified as gifted, to understand and learn from the ways they maneuver and make sense of their experiences within the school setting. Ford and Whiting (2010) noted that African Americans live and learn in school communities that do not adequately recognize or value the worth of their ethnicity. The researchers stated that "many [African Americans] struggle to develop a positive sense of self, which includes views about their personal worth, their academic worth, and their social worth as racial beings” (p. 149). CRT then, in addition to promoting the validation of worth through exposing lived experiences, is an appropriate conceptual framework through which to conduct a phenomenological study because "the voice component of critical race theory provides a way to communicate the experience and realities of the oppressed" (Ladson-Billings \& Tate, 1995, p.58). Understanding the experiences of gifted African American males in a gifted program, through their own voice, gives a first-hand account of the unique attributes of their experiences that account for the phenomenon under investigation. It also provides a platform, not just for their experiential storytelling, but their counter-storytelling of persistence, resilience, high self-efficacy, and achievement amidst discreet undertones and blatant overtones that exist in the racially laced constructs of the education system.

\section{Group and Deficit Thinking Theory and its Application to African American Male Enrollment in Gifted Programs}

Ford, Moore, and Scott (2011) purported that "deficit thinking is the major reason gifted education underrepresentation exists, persists, and is also extensive or pervasive” (p. 240). Valencia and Solóranzo (1997) described deficit thinking as the 
notion of placing blame on marginalized groups, such as those of low socio-economic status, and racially and culturally different, for their own social and academic outcomes. While blame falls squarely on these groups, it is dismissive of any contributing social or systemic inequalities (p. 10). The cognitive and behavioral shortcomings associated with deficit thinking implicate students of color as being responsible for their academic underachievement and social dysfunction without complicity from external forces (Olivos, 2006). Moore, Ford, and Scott (2011) reported that "when deficit thinking exists, educators perceive African American students to be genetically disadvantaged or culturally disadvantaged; some even believe African Americans to be less capable than other students” (p. 241). More specifically “in gifted education, deficit thinking can take (and has taken) the form of either teachers not referring or under-referring African American students for gifted education screening, identification, and placement” (Ford \& Grantham, 2003, p. 224). The researchers also asserted that, "until deficit thinking becomes dynamic thinking, the unnecessary underrepresentation of diverse students in gifted education will continue” (Ford \& Grantham, 2003, p.217). Essentially, positive narratives and images that affirm the capabilities and strengths of gifted African American males must replace deficit thinking.

The toxic implications of Deficit Thinking theory can be conceptualized through the lens of Allport’s (1954) theory of prejudice. Allport (1954), as cited by Ford, Moore, and Scott (2011), "identified five degrees of prejudice: antilocution, avoidance, discrimination, physical attack, and extermination” (p. 241). Allport (1954) provided examples of how each of the degrees of prejudice, with the exception of extermination and physical attack, manifests itself in gifted education. Ford, Moore, and Scott (2011) 
explained that antilocution encompasses any negative comment made by an educator about a gifted African American student. Avoidance takes the form of educators segregating gifted Black and White students in the classroom. Discriminatory practices perpetrated by educators denies equal learning experiences and opportunities to gifted African American students in the same way they are afforded to White gifted students. The terms "physical attack and extermination are self-explanatory", not requiring further elaboration based on the explicit inference of the terms themselves (Ford, Moore, \& Scott, 2011, p.242).

While the Deficit Thinking Theory provides clarity on the behavior of educators in their thinking toward gifted African American students, voluntary and involuntary minority groups theory illuminates the thinking behind the perceptions of majority and minority groups and examines why those perceptions exist. Ogbu and Simmons’ (1998) theory of voluntary and involuntary minority groups' makes distinctions between the historical and cultural experiences of different groups in the United States. Voluntary groups made purposeful decisions to immigrate to the United States with the expectation of having a prosperous life. Involuntary minority groups, such as slaves, were brought to the United States against their will in depraved circumstances and forced to assimilate (Ogbu, 1992). As a result, the involuntary group and descendants of that group, have become resistant, angry, and rejected the values, traditions, customs, and behaviors of the new culture (Simmons, 1998). The latent impact of voluntary and involuntary groups is evident in today’s classroom in that "when African American students are angry, resentful, or even hostile toward following the traditional ways of being and behaving in school settings, it is not likely that someone would see them as gifted or capable of 
succeeding in gifted education” (Ford, Moore, \& Scott, 2011, P. 244). This means that disposition alone should not be used as a criterion by which educators dismiss the potential for giftedness.

Gifted African American males can thrive academically in gifted programs in the same manner as their White counterparts. The benefits of gifted programming coupled with empowerment strategies formulate an academically and socially healthy learning environment. Ford, Grantham, and Bailey (1999) found that having a racially, ethnically, and culturally diverse gifted student population increases the likelihood that African American males are motivated and interested in participating in a gifted and talented program. This suggests that the representation of non-minority students in gifted programs is essential to establishing the environmental conditions that enable African American males to prosper academically.

\section{Summary of the Literature Review Findings}

The literature is clear with regard to the influence of racially driven practices and deficit ideology perceptions that limit the enrollment of gifted African American males in gifted programs. As noted in the literature, the marginalization of gifted African Americans is evidenced through underrepresentation in gifted programs. The issue of underrepresentation is even more pervasive among gifted African American males due to a host of social and academic barriers normalized and accepted as a part of the African American educational experience. Specifically, underrepresentation is a dysfunction of recruitment, identification, and retention practices that ignore, undervalue, and discredit the intellectual capabilities of gifted African American males. 
The preponderance of literature regarding the enrollment of African American males to gifted programs contests: (a) the current lens through which giftedness in conjunction with cultural factors are interpreted among gifted African American males: (b) challenges bias assessment instruments used to measure giftedness, and (c) questions the stagnant development of an affirming social context in which they can thrive. The literature also expounds upon the role of teachers as gatekeepers to gifted programs and the cultural disconnect barricading gifted African American males from accessing gifted services. Relevant research regarding the role of self-efficacy among gifted African American males, as it relates to academic achievement, indicates that a scholar identity stimulates the demonstration of high self-efficacy among gifted African American males and positively impacts academic achievement.

Critical Race Theory (CRT) presents a useful framework in deconstructing the universality of the racial undertones that are harmful and unproductive in educating gifted African American males. Voluntary and Involuntary Groups theory, coupled with Deficit Thinking theory, provide a historical and modern basis for interpreting the reasons barriers exist for gifted African American males who qualify to participate in a gifted program. Furthermore, according to Borland, Schur, and Wright (2000) the underrepresentation of minority students and students from economically disadvantaged backgrounds in gifted programs is one of the most disturbing and recalcitrant issues facing gifted education in the U.S. This mindset indicates that gifted African American males are at increased disadvantage for gifted programs based on what should be irrelevant factors beyond their control. Findings from the literature also suggest further 
action to reverse the deluge of academic and social barriers that are resistant to changes in policy and practice related to gifted African American males.

The literature referenced in this chapter contribute to exposing and explaining the needs of gifted African American males, but minimally confronts the myriad of challenges faced by gifted African American males in gifted programs in comparison to the enormity of relevant topics yet to be studied. The qualitative and quantitative methodologies used in the studies of gifted African American males allows researchers to examine the depth and breadth of the multi-faceted elements of this student population. The literature does however suggest that more research is needed to expound upon the barriers encountered by gifted African American males to validate and amplify how their experiences influence self-efficacy and academic persistence (Whiting, 2009).

Considering the extant research in this field, there are still gaps in the literature in this field of study. In general, Henfield, Moore, and Wood (2008) claimed that literature regarding gifted African American males is sparse. Thomas (2009) posited that retention of African American males is a serious issue and few studies examine the factors that affect retention in gifted programs. While the studies of this literature review do not exhaust the extant research, the pool of literature is still limited and may speak to why discussions of underrepresented minority populations in gifted programs from 20 years ago are still being rehearsed today (Ford, 2010). 


\section{CHAPTER III}

\section{METHODOLOGY}

\section{Introduction}

The purpose of this research study was to explore the lived experiences of gifted African American males in Grades 6-8 participating in a gifted program and to provide insights into how their sense of self-efficacy influences academic persistence. An exploration of the experiences of gifted African American males participating in a gifted program has the potential to initiate dialogue and corresponding action aimed at counteracting the virulent practices associated with the identification of underrepresented populations in gifted programs, particularly African American males. Additionally, close examination of their experiences serves to inform and engage practitioners and policymakers in reformulating the programmatic needs of gifted minorities. This study is needed to aid efforts to change the educational trajectory of gifted African American males because "many advocate for the rights of gifted students in general, but rarely fight for those who have different needs...[therefore], when addressing inequities in gifted education, underrepresentation cannot be ignored” (Ford, 2014, p. 144).

This study used a qualitative research approach. This chapter provides a detailed explanation of the research study design, the theoretical approach and its applications, sampling and site selection, data collection, data analysis, implications and ethical 
considerations, an explanation of the role of the researcher, and the study's limitations. The study will examine the following research questions:

1. How do gifted African American males understand and experience participation in a gifted program?

2. How do African American males perceive their participation in a gifted program as shaping their sense of self- efficacy and academic persistence?

\section{Research Design}

Qualitative research is a nonstatistical method of inquiry and analysis of phenomena occurring within a social context in which the intentional and purposive selection of participants ensures the relevance of their experiences to the study (Gall, Gall, \& Borg, 2007; McRoy, 1996). It explores the studied social context and its content to extrapolate understandings and experiences by deciphering societal norms, gauging the influence of verbal and nonverbal discourse, institutionalized processes, and the dynamics of relationships, in order to articulate derived meanings (Mason, 2013; Schwandt, 2005). Aptly, the qualitative researcher conducts studies in the participant's natural setting and seeks to make sense of and interpret the phenomenon under investigation. The intent of qualitative research is not to generalize findings based on the attributes of singular or collective experiences; rather it is interested in the applicability of findings based on how the experiences themselves are transferable. In other words, "the aim is to make logical generalizations to a theoretical understanding of a similar class of phenomena” (Popay, Rogers, \& Williams, 1998, p. 348).

The qualitative researcher utilizes representations such as participant observation, field notes, interviews, focus group discussions, photographs, memos, and recordings as 
sources of data (Denzin \& Lincoln, 2008; Fossey, Harvey, McDermott \& Davidson, 2002). The qualitative analysis produces rich and descriptive data interpreted by coding, categorizing, sorting and extracting textual and thematic trends denotative of significant findings to inform both practice and theory (Boeije, 2010). This process allows the researcher to move from data collection to constructing explanations and interpretations of the specific subjects under investigation (Gibbs, 2002).

The flexibility of qualitative research is inherent to its emergent design, meaning the researcher does not ascribe to presupposed outcomes and is able to focus on both the research process and its results (Hoepfl, 1997). The flexibility of qualitative research does not diminish its credibility as a scientifically rigorous method; in fact, the amplitude of its flexibility reflects the depth of understanding through analysis. Unlike the discrete data collection methods of quantitative research, qualitative research methods allow the researcher to explore open-ended questions and prompts to probe and encourage participants to elaborate on their responses (Mack, Woodsong, MacQueen, Guest, \& Namey, 2005). Furthermore, Mack et al. suggested that the exploratory nature of qualitative research elicits responses from participants that are "meaningful and culturally salient to the participant, unanticipated by the researcher, [and are] rich and explanatory" (p.4). Denzin and Lincoln (2000) noted that phenomenology captures the essence of qualitative research. Since qualitative research is effective in capturing the cultural nuances influencing perceptions, values, behaviors, and the experiences of specific populations in a particular social context (Mack et al., 2005), it is fitting then, that phenomenology may be used in the context of the current study. 


\section{Phenomenological Approach}

Founding phenomenologists Edmond Husserl and Martin Heidegger established the philosophical premise of phenomenology as an inductive research method for understanding and constructing meaning from experiences (Van Manen, 1997). Van Manen further explained that phenomenology reveals the realities of a lived experience "rather than how it might be explained or described in theoretical terms, and it involves modes of discourse, voice, and expression that can reveal felt meaning that goes beyond the prevailing paradigm of logic, prediction, and control” (p. 27). Concisely stated, phenomenological research aims to study how humans cognitively and perceptually experience phenomena (Wilson, 2002). It also provides a way of thinking about and studying social realities (Strauss \& Corbin, 1998). Based on the purpose and attributes of phenomenology, a phenomenological study was an appropriate method to examine the unique experiences of gifted African American males, because phenomenology "is an attempt to deal with inner experiences unprobed in everyday life” (Merriam, 2002, p. 7). As such, this phenomenological study determined how gifted African American males perceive their lived experiences in a gifted program as shaping their sense of self-efficacy and academic persistence. Examining gifted African American males' participation in a gifted program provided insight into emergent patterns representative of their lived experiences and how they constructed meaning from those experiences.

Sokolowski (2000) characterized phenomenology as involving thick descriptions and close analysis of lived experience to understand intimately how subjects create meaning through their perceptions. In addition, phenomenology contributes to a rich understanding of lived experiences often overlooked or taken-for granted that reveal 
ways in which we know and understand (Stewart \& Mickunas, 1974). Using verbal, textual, and visual data, interpretation and analysis enables the researcher to uncover a description of the 'essence' of the phenomenon (Petty, Thomson, \& Stew, 2012). To analyze the descriptive data of a phenomenological study, the researcher must determine whether the data will be filtered through the descriptive or interpretive philosophical school of phenomenology (Lopez \& Willis, 2004).

Descriptive phenomenology, attributed to Husserl, is based on the belief that the researcher must actively suspend all suppositions, personal biases, and consciousness of prior expert knowledge to derive the essence of the phenomenon (Natanson, 1973). Heidegger, modified and built upon Husserl's seminal ideology, identifying hermeneutics or interpretive phenomenology as a method for revealing what is normally concealed in the human experience (Spielberg, 1976). Heidegger asserted that it is impossible for the researcher to disregard their personal experiences related to the phenomenon under study, and to do so would devalue the personal awareness of the researcher which is intrinsic to phenomenological research (Reiners, 2012). Heidegger, subscribed to the concept of coconstitutionality, meaning the work of an interpretive researcher is an amalgamation of the meanings articulated by the researcher and participant (Koch, 1995).

In short, Hermeneutics goes beyond descriptions of the way individuals conceptualize experiences, to examining meanings embedded in those experiences because meanings are not always discernible to the participant, but extracted from their personal narratives (Pietkiewicz, Smith, 2014; Solomon, 1987). Hermeneutics also satisfies the need to understand the mindset and language of a person to interpret the manner in which they mediate their experiences in the world. (Freeman, 2008). As such, 
Heidegger (1962) was concerned with the ontological position of the existence of self as explained through the concept of lifeworld and situated freedom.

Specifically, Heidegger used the term lifeworld to convey the idea that the realities of an individual are shaped by the world in which they live with the understanding that an individual cannot detach themselves from life experiences. Moreover, the inspection of the lifeworld of participants in a group under investigation examines the extent to which they contribute to commonalities in and differences between experiences (Lopez \& Willis, 2004). Therefore, the essence of hermeneutic interpretive inquiry gives credence to what is implied in the narratives emanating from life's daily experiences. That is to say, interpretive phenomenology encourages participants to reflect upon and describe interactions and relationships with others to situate their lived experiences in the context of daily routines, practices, and socialization. Furthermore, interpretive analysis within the context of phenomenology may involve acknowledgement of the historical, social, and political elements that construct experiences (Smith, 1987). Based on the attributes of interpretive phenomenology, this methodological approach provided an appropriate schema through which to scrutinize carefully the perceptions of gifted African American males. Additionally, interpretive phenomenology expands upon superficial understandings of their experiences to more deeply understand and discern what it means to be a gifted African American male in a gifted classroom and school setting in general.

\section{Application of Theory}

I used Critical Race Theory (CRT), Deficit Thinking, and Voluntary and Involuntary Groups to inform the data collection and analysis process. I employed semi- 
structured interviews and focus groups, utilizing photo elicitation, to demonstrate the ways in which the theoretical frameworks shape the design of the study. CRT encompasses four tenets. First, CRT propounds that race is experienced and understood differently by different racial groups based on their social context. Second, it posits that the racial experiences of minority groups are subservient to the White racial experience. Third, it expounds on how race camouflages societal norms to appear unbiased, when in fact, race methodically places minority groups at a significant disadvantage (Davis, Johnson, \& Martinez, 2001; Delgado \& Stefancic, 2000). Finally, CRT offers a theoretical explanation for the enduring racial inequalities and social injustice that exists beyond the Civil Rights Movement and are accepted as a justified societal norm.

Based on these assertions, CRT was an appropriate framework through which to examine how gifted African American males understand and experience their participation in a gifted program. As such, interview questions 1-6 (see Appendix C) are constructed to extract participants' rich descriptions of experiences and how they make meaning of those experiences. Since CRT posits that race is experienced and understood differently by different racial groups, interview questions such as, "What have your experiences been as a participant in a gifted program?" and "What makes you feel accepted or not accepted in this group?" address how the experiences of gifted African American males are influenced by race and racism normalized in the education pipeline. Subsequent interview questions addressed how African American males' perceptions of their participation in a gifted program shaped their sense of self-efficacy and academic persistence. Solomon, Portelli, Daniel and Campbell (2005) asserted that for public school students, myths of racial inferiority that are prominent in curriculum and teacher-student interactions drive beliefs 
about the inferiority of Black students, making it difficult to disrupt the primacy of race in school settings. Therefore, posing the questions, "Please describe what being gifted means to you?" and "Have you ever experienced discrimination in your school environment?" confronts the implications of self-perception and the racial disparities that exist in the construction of school and classroom norms. The questions also address the implications of how gifted African American males perceive their experiences from the perspective of race and its influence on their sense of self-efficacy.

During the data analysis process, CRT, in alignment with the study's research questions, was instrumental in the development of a start list to initiate the coding process. This process guided the parceling out of attitudes, dispositions, and perceptions of gifted African American males’ participation in a gifted program (Miles, Huberman, \& Saldaña, 2014). The start list included codes such as, 'feelings', 'classroom experiences’, 'racism', and 'self-efficacy'. Other codes emerged inductively throughout the data collection process (Miles, Huberman, \& Saldaña, 2014).

Whereas CRT focuses on race as an institutionalized and endemic facet of society, deficit thinking and voluntary and involuntary groups theories aided in narrowing the focus of this study to examine the factors influencing how minorities themselves experience education. Generally, deficit thinking refers to the misappropriated blame that places perceived deficiencies, low expectations, and academic underachievement on the group in question, rather than as a function of cultural and societal structures (Ford \& Grantham, 2003; Olivos, 2006; Valencia, 2010). In the current study, understanding the effects of deficit thinking provided salient explanations during data collection and analysis offering insight into how gifted African American males mitigated the effects of 
deficit thinking and chose to remain in a gifted program. For example, the prompt"Describe what makes you feel that you can be successful in a gifted program" may reveal participant's thoughts on teacher expectations and the extent to which they perceive their teachers believe they can be successful regardless of race. Emergent themes during coding were used to identify phenomena linked to deficit thinking and its latent effects on gifted African American males’ participation in a gifted program.

Analysis of data related to deficit thinking resulted in greater understanding as to why deficit thinking is the major reason underrepresentation exists in gifted programs (Ford, Harris, Tyson and Trotman, 2002). Likewise, participant responses to, "Describe how you feel when you are presented with a new or challenging task in class? or "Describe how you overcome obstacles you encounter in order to do well academically?" sheds light on the extent to which gifted African American males' sense of self-efficacy propels them to persist academically amidst deficit paradigms. The questions probe the participant's confidence in completing tasks and persisting in the face of academic and social adversity. The questions also provide an opportunity to explore factors influencing self-efficacy such as stereotyping and negative teacher perception that could affect academic persistence (Uwah, McMahon, \& Furlow, 2008).

Similarly, voluntary and involuntary groups theory was applied to interpret the academic aversion experienced by gifted African American males in conforming to the norms that impact academic success. Voluntary and involuntary groups theory, describes the orientation and approaches of specified groups toward their acceptance of societal norms and behaviors based on the way in which they are introduced to a new culture (Ford, Moore, \& Scott, 2011). Ogbu and Simmons (1998) theorized that voluntary 
minorities have had different historical and cultural experiences in the United States, therefore favorable social and academic outcomes are expected. In contrast, involuntary groups, such as slaves and subsequently their decedents, experience the United States through the lens of anger and resistance as a result of a legacy of oppression. Therefore, the disposition of gifted African American males can be assessed in the prompt, "Have you ever wanted to be exited from the gifted program? Describe the circumstances that made you feel that way.” This inquiry, for example, may capture experiences that trigger feelings of resentment, displacement, rejection, or isolation and give clarity to their acceptance, conformity, or lack of conformity to social norms in a gifted program. In the same way, focus group discussion prompts (see Appendix D), and the utilization of photographs (see Appendix E) to elicit participant responses, are aligned to address the problem under investigation. For instance, during the focus group discussion, participants were asked, "Did any of the photos cause you to have specific memories of your experiences in school?” This line of questioning, not only addresses the issue of how gifted African American males understand and experience participation in a gifted program, it also presents an opportunity for participants to engage in counter-storytelling, which is inherent to CRT. According to CRT scholars Delgado and Stefanic (2002) counter-storytelling "help[s] us understand what life is like for others, and invite[s] the reader into a new and unfamiliar world” (p.41). Furthermore, sharing accounts in their own words, challenges the discourse of elitism and privilege often encountered among White students (DeCuir \& Dixson, 2004).

In general, the selected theoretical frameworks provided a foundational lens through which to grasp the ideological influences of race and its impact on the practices 
that promote the underrepresentation African American males in gifted programs. The frameworks also established the foundation for the development of an academic and culturally conscious blueprint for systemic change based on the marrying of theory, data collection, and analysis protocols. Interviews and focus groups captured the essence of the lived experiences of gifted African American males, while the analysis of textual data uncovered phenomena grounded in each framework. The interplay of the applied theoretical frameworks and the analysis of data serve to support the research findings which are explained in Chapter V.

\section{Site Selection and Sampling}

The district that serves as the context of this study is one of the largest urban school districts in the United States. The total current student population in the school district is 101,328 . The demographic composition of the district is $51 \%$ white students, 36\% African American students, 7\% Hispanic students, and 6\% accounts for other races. There are 91 elementary schools, 27 middle schools and 21 high schools. Eighty-six of these schools are schools of either high or extreme poverty as noted in the district's equity report. African American males make up 18\% of the district's total enrollment. While African American students make up 36\% of the total student population, they represent only $8 \%$ of the total enrollment in advanced classes, some of whom are considered gifted (Data Books, 2015).

Purposive sampling was used to select the participants for this study. Purposive sampling methods are often used in qualitative research studies so that the participants have relevant experiences in the phenomenon being studied. Creswell (2009) 
recommended selecting participants who can provide essential information about the phenomenon being studied. According to Moustakas (1994), phenomenological research is derived from first-person reports of lived experiences. Phenomenology describes the meaning of experiences lived by several individuals and seeks to understand the nature of those experiences (Hatch, 2002), therefore participants selected attended an urban middle school (Grades 6-8) which had a district approved gifted program or gifted magnet. At the time of the study, there was only one official gifted magnet program for Grades 6-8 in the urban school district that served as the context for the study. The purposively selected participants included all 11 African American male students identified as gifted and participating in this gifted program.

The school district uses the Cognitive Abilities Test (CogAT) to determine giftedness. The CogAT measures students learned reasoning abilities in the three areas most linked to academic success in school: Verbal, Quantitative, and Nonverbal domains (Lohman \& Hagen, 2005). Based on the school district guidelines, students must earn a minimum of a 24 on a 27-point scale on the CogAT to be identified as gifted. Based on the criteria used to identify students as gifted, it is worth noting that African American students as a group have never achieved test scores comparable to their White counterparts in similar educational contexts (Helm, 2008). Disparities in test scores may be attributed to the fact that African Americans have minimal contribution to the formulation of assessment structure or its content, yet African American test takers are expected to perform to the same level of achievement as White students without consideration given to assessment cultural bias (Ford \& Helms, 2012). Ford and Helm found that although qualitative and quantitative investigations of the existence of cultural 
bias in test instruments is well documented and validated, transitions toward developing assessments void of cultural bias as a means of providing access to African American has been negligible.

Admittedly, the district-based criteria used to select participants for the current study is limited in identifying African American males who may be gifted, but failed to obtain the designated score to qualify as gifted for this gifted program. In order to adhere to the school district's benchmark for giftedness and to provide an objective measure to track the achievement of African American males in the gifted program for future studies, African American males who are "sit-ins", meaning, they do not have the required score to be considered gifted, but participate in gifted classes, were excluded from this study. Furthermore, it is unclear if students classified as sit-ins receive all of the benefits afforded to students officially identified as gifted in this gifted program.

Letters explaining the purpose and scope of the study to include parent consent forms (see Appendix A) were sent to the parents of the participant's meeting the criteria and who were enrolled in the gifted program. I made follow-up phone calls to each parent to ensure the letter was received. Parent consent forms explained the purpose and intention of the study and contained language aligned with the Assent forms. The parent permission forms were obtained before research began, even if Assent forms from participants were received first.

In accordance with the Institutional Review Board (IRB), participants were guided through all stages of this study during a research study orientation. Participants were required to review and sign assent forms before any data was collected (Creswell, 2014). Since minors are the subject of this study and are considered vulnerable subjects, 
special protocols were followed. According to the Federal Policy for the Protection of Human Subjects, (2001) “children are considered vulnerable, in part because of the legal limitations on their autonomy, but also because of their presumed reduced capacity to understand and fully participate in the informed consent process” (p.17). To ensure the participants were fully knowledgeable of the research objectives of this study, they received an assent form (see Appendix B). The assent from provided a clear explanation of what the study was designed to achieve, the purpose for which the study was being conducted, their role in the study, and the utilization of the findings. The document informed the participant of their right to discontinue involvement at any time without any repercussion should they ever feel harmed by the process (Glesne, 2016).

After obtaining permissions from the study site, a list of potential subjects was requested. Verification of ethnicity was based on how the participant's race identified them in the school's enrollment database. Identification of race was determined by their parent or guardian's selection for race on the district's enrollment form. In consideration of the sensitive information that may be uncovered in the findings of this study, participants were given the option of assuming a pseudonym.

During the orientation meeting inclusive of participants and their parents, the agenda consisted of: (a) an overview of the study; (b) instructions regarding Consent and Assent forms; (c) interview times scheduling; (d) the focus group meeting scheduling; and (e) the participant incentive details.

\section{Data Collection Methods}

For the current study, I utilized two data sources. Participants engaged in a semistructured interview lasting 45-60 minutes. Semi-structured interviews are a primary 
mode of data generation in phenomenological studies (Reiter, Stewart, \& Bruce, 2011). Wimpenny (2000) explained that a "phenomenologist often commences an interview with "Please describe your experiences of... with clarification sought to enrich the description and illuminate that experience” (p. 1489). Reiter, Stewart, and Bruce (2011) suggested that interview questions should not impose any particular stance to the interviewee. Following this model, face-to-face interviews were conducted with a semistructured approach and recorded using a recording device. According to Giorgi, (2009) "what one seeks from a research interview in phenomenological research is as complete of a description as possible of the experiences that a participant has lived through” (p.122). Interviews were conducted during the school day at the selected site during the HomeRoom period. This period was selected to prevent the participants from missing any core content classes and to capture the authentic experiences of the subject within the context of the academic environment.

Prior to the interview, time was given to the participants to review the 11 interview questions (see Appendix C). The purpose of the question preview was to allow the participants to feel comfortable with responding to the interview questions. Interviews were recorded and transcribed for later analysis. All interview recordings were locked in a file cabinet to ensure the security of the interview content and to protect the confidentiality of the participants.

In addition to semi-structured interviews, I conducted two focus groups, utilizing photo elicitation to gather textual data from visual cues. Focus groups are a data gathering method using semi-structured and in-depth group interviews. Focus groups have evolved beyond the field of market research to becoming a desired method to 
examine people's experiences and attitudes in academic and applied domains (Colucci, 2007). Focus groups are unique in achieving rich group interactions and understanding social constructs (Green, Draper, \& Dowler, 2003). Participants are selected purposively to discuss issues based on themes decided upon by the researcher that relate to the topic under investigation (Kumar, 1987; Lederman, 1995). They are also selected based on their 'applicability', meaning that they are selected because of their knowledge and experiences on the topic of study (Burrows \& Kendall, 1997, p. 247). Focus groups are unique in that data is collected from multiple subjects simultaneously. However, rather than the researcher obtaining data directly from the participants, they are encouraged to talk to each other, ask questions, and describe their experiences and personal perspectives (Kitzinger, 1995).

Smaller homogenous groups are ideal for focus group discussions. Krueger and Casey (2000) suggested that six to eight participants are optimal to solicit full participant engagement. Kitzinger (1995) found that focus groups helped people to "explore and clarify their views in ways that would be less accessible in a one-to-one interview" because participants are able to explore the issues of importance to them, in their own vocabulary, generating their own questions and pursuing their own priorities” while providing mutual support (p.29). Additionally, focus groups promote sharing ideas and feelings, enabling the researcher to make distinctions between individual versus group perspectives (Rabiee, 2004). Due to the participatory nature of focus groups, copious amounts of data are generated in a short period of time, therefore it in incumbent upon the focus group moderator to have pre-arranged protocols to structure the discussion. 
In order to maximize the benefit of focus group discussions, Kitzinger (1995) proposed that focus groups should be conducted in a comfortable environment to create a safe space for a candid exchange of ideas. Escalada and Heong (1997) suggested that the moderator should organize seating arrangements, distribute name tags, and allow for one to two hours for activities and discussion. He promoted the necessity for the moderator to record the session for transcription and to be vigilant in documenting nonverbal expressions, which provides as much pertinent data as verbalization. Gill, Stewart, Treasure, and Chadwick (2008) offered that moderators must guide the discussion without participating, to avoid their personal views to influence participant responses and interactions.

All of the purposively selected subjects were invited to participate in the focus groups. A focus group protocol (see Appendix D) was used to ensure consistency between the two groups. Each group had five participants and convened after school in the Media Center at the selected site. Participants received name tags to aid in being able to identify which participants were contributing to the discussion. Although open-ended questioning is commonly used to generate discussions in a focus group, I moderated the focus group discussion using four photographs (see Appendix E) which depicted various aspects of educational experiences, intentionally selected to elicit responses from the participants. I used the free listing technique, which is frequently used to examine cultural domains (Bernard, 1995). Bernard described 'free listing' as a process by which pertinent words and phrases are generated and listed to observe thematic trends. Participants generated words and phrases that captured their interpretations, thoughts, and feelings, invoked from viewing the photographs while I recorded them on large chart 
paper. Bartunek and Seo (2002) recommended that the moderator check for frequency in the reoccurrence of words or themes to gain insight into the salience of specific attributes to the group. Therefore, I used symbols to notate repetitive elements. Participants discussed how the terms they listed described or were relevant to their classroom experiences. The focus group protocol incorporated interrogative prompts such as- "How does the image make you feel?” or “Do any of the images remind you of your own experiences?” The free lists were coded in the data analysis process and focus groups discussions were recorded for transcription and coded.

The utilization of photo elicitation during the focus group captures a dimension of data that may otherwise go unexplored. Photo elicitation was first debuted by Collier (1957) as a means of inserting photographs into research interviews. The scientific basis for the significance of photo elicitation relates to brain function. The sections of the brain that make sense of images are more developed from the standpoint of physiological evolution, than the sections of the brain that process verbal information (Harper, 2002). Harper asserts that images more easily inspire deeper levels of consciousness than words.

As such, Collier (1957) proposed that photo elicitation prods latent memories and spurs emotional liberation as evidenced in the statements participants' shared about their life's experiences. Several studies have used photo elicitation to explore social organization, social class, community, identity, and culture (Craig, Kretsedemas, \& Gryniewski, 1997; Curry, Strauss, \& Strauss, 1986; Guschker, 2000; Harper, 2000; Kretsedemas, 1993; Sampson-Cordle, 2001). Specifically, the studies expanded upon the knowledge on social identity and culture through the interpretation of what is depicted in an image. Most elicitation studies use photographs, but many use paintings, cartoons, or 
public displays selected by the researcher. Hall, Jones, Hall, Richardson, and Hodgson (2007) found that although photographs generate narrative directly from an image, they also produce powerful descriptive reflections that may be unrelated to the displayed image yet are representative of the subject's personal experiences. Based on the extensive uses of photo elicitation and its universal utility, Hall et al. (2007) contended that "the use of photo elicitation techniques has overall, provided a positive appearance as a highly effective, useful and reliable method for use in research” (p.3). For the current study, the four preselected images were intended to stimulate relevant responses to the participant's experiences. Harper (2002) suggested that these photographs should capture "work, school or other institutional experiences, or images depicting events that occurred earlier in the lifetime of the subjects. The images may connect an individual to experiences or eras even if the images do not reflect the subject's actual lives” (p. 13). In consideration of Harper's suggestion, the photographs were found online and chosen based on their pertinence to the research questions addressed in the study and for their ability to capture the African American male experience in the classroom. Harper also suggested that images could be created by the researcher or the subject during research. Therefore, after participants exhausted their free lists, they were prompted to create an image depicting their thoughts, apprehensions, or desires as it relate to their experiences in a gifted program or that reflect their schooling experiences in general.

\section{Data Analysis}

Rubin and Rubin (2005) explained that the purpose of data analysis is to understand concepts and recognize emerging themes that are descriptive of the topic being examined. The data analysis process brings order, structure, and a system for the 
interpretation of the data being collected (Marshall \& Rossman, 1999). Although data are collected at various points throughout the research process, simultaneous analysis of the data enables the researcher to focus and shape the study as it progresses (Glesne \& Peshkin, 1992). In the current study, data sources were analyzed to respond to the study's research questions by extracting meaning from the experiences of gifted African American males, observing thematic trends, and rendering interpretations through analysis processes appropriate for interviews, photo elicitation, and focus groups.

Originating within the methodology of phenomenology, Tufford and Newman (2010) described bracketing as a method used to lessen the potential damaging effects of preconceptions not recognized by the researcher related to the research topic, thereby increasing the accuracy of the study. Heidegger however (1962) argued that in order to fully understand lived experiences, bracketing out preconceptions was impractical and not favorable (LeVasseur, 2003). Heidegger (1962) further asserted that the position of the researcher brought meaning and valuable interpretations to the research that might not otherwise be realized (Gering, 2004). In the current study, bracketing was not used. Ascribing to the assertion of Heidegger that the researcher cannot be disconnected from the meaning extracted from the participant's experiences and essentially becomes a part of the phenomenon, my position is deemed essential in facilitating a dual interpretive process. That is, the participant makes meaning of their world and the researcher decodes that meaning to make sense of how the participants have made meaning from that world (Smith \& Osborn, 2007).

Interpretive phenomenology researchers extract meaning from participants' experiences during the analysis process by posing questions of the data such as: "What is 
the person trying to achieve here? Is anything meaningful being said here, which was not intended? Do I have a sense of something going on here that the person himself or herself is perhaps less aware of?” (Pietkiewicz \& Smith, 2014, p. 8). According to Pietkiewicz and Smith, such interpretive inquiry leads to a more rich and comprehensive analysis and drives an in-depth examination of the phenomena under investigation. By extension, the emphasis of interpretive phenomenology on sense-making by both the participant and researcher gives credence to the role of cognition as being central to the analytic process. Interpretive phenomenology draws upon the subject's cognition, language, affective and physical state, and acknowledges the complex connections between what the subject says, their thinking, and emotions (Smith \& Osborn, 2007). The data is then analyzed through extensive engagement with the text derived from participants.

In the current study, interviews were recorded and transcribed. Interview summaries were written after each interview to note any extraneous information not captured in the recording, such as body language and gestures (Yin, 2003). The interview transcription was used to initiate a coding process. Trede and Higgs (2009) asserted that selecting a coding method that addresses a participant's lived experiences and realities may be better at revealing those ontologies. Therefore, the ontological research questions posed in the current research study influenced coding choices. Coding was utilized to assign as many categories as possible to the text by labeling the interview transcription (Glaser, 1992).

Coding is the use of words or phrases that provides the summative essence for a section of text or a visual image (Saldaña, 2016). This process helps the researcher to group text with similar codes based on shared characteristics (Bernard, 2011). A "start 
list” was used to initiate the deductive coding process (Miles, Huberman, \& Saldaña, 2014). It was necessary to engage in more than one cycle of coding, and the sorting of codes, for generating categories, themes, concepts, and meaning (Coffey \& Atkinson, 1996). Categories created from the synthesis of codes are expected to become more defined as the process of recoding and recategorizing takes place (Punch, 2009). For the current study, I manually coded semi-structured interview and focus group discussion transcript, free listing charts, and interpreted participant generated images in order to preserve the data as close to the substance of the participant's own wording and sentiments as possible.

Modeling Smith and Obsorn’s (2007) interpretive analysis protocol, initially I performed a close reading of the interview transcript multiple times to become immersed in the data. The left-hand margin of the transcript was used to annotate observations and reflections about the interview or other thoughts of significance to the study. Annotations included summarizing or paraphrasing what was discussed, the manner in which participants used language features such as metaphors, colloquialisms, symbolism, contextual elements, and preliminary interpretations. Descriptions giving insight into the emotion of the participant were highlighted in the transcript. The notations allowed me to convert notes to emerging themes.

Secondly, I toggled between important themes generated during the analysis process connecting themes to specific subject narrative while comparing and contrasting them (Pietkiewicz \& Smith, 2014). Connections between emerging themes were extracted, grouped by similar theme, and a descriptive label assigned to each cluster. Some themes were eliminated if they had loose or weak associations with the emerging 
thematic structure. Finally, based on emergent themes, I examined subordinate themes and subthemes. Although interviews were recorded and transcribed, I used member checking, which will be explained in the next section, to allow participants to check transcripts for inconsistencies and to serve as a means for further clarification.

The research design of photo elicitation recognizes the participant as a source of data, but also as a source of the emergent data analysis (Jenkins, Woodard, \& Winter, 2008). The analysis of visual material can present challenges in terms of how to communicate and report on the data being collected, "however there is an intrinsic value in photographic content generated by users which can effectively be fed into the research design, without the specific need for complex and largely unnecessary visual data analysis” (Hall, Jones, \& Hall, 2007, p. 227). Gibson and Brown (2009) stated that visual data should be analyzed through both a holistic and interpretive lens propelled through inquiry. The researchers suggested that while still appropriate, instead of fully relying on singular words or short phrases, the researcher should make use of field notes and analytic memos to find meaning from the text provided by participants. Since interpretations and analysis of visual images should produce rich descriptions, Gee (2011) affirmed that methods used for discourse analysis, such as semi-structured interviews, are just as appropriate for analyzing visual data. Therefore, the participants' reflective reactions to photographs mounted on the chart paper were coded, sub-coded, categorized, and sorted similar to the process described for the analysis of the semistructured interviews. For each of the data sources, I determined the saturation point as being when "no new or relevant data seem to emerge regarding a category, the category 
is developed in terms of its properties and dimensions, and the relationships among categories are well established and validated” (Strauss \& Corbin, 1998, p. 212).

Finally, a framework analysis, akin to Smith and Osborn’s (2007) interpretive analysis protocol, was used to analyze the focus group discussion. Framework analysis involves the following five distinct, yet associated phases: (a) familiarization, (b) identifying themes, (c) indexing, (d) charting, and (e) mapping and interpretation (Ritchie \& Spencer, 1994). During familiarization, I listened to the recordings, read the transcripts several times, reviewed observational notes, and summaries taken during and after the discussion to become fully immersed in the details. Themes were identified by writing words and phrases in the margins of the transcript leading to the surfacing of concepts and ideas. Indexing involved isolating and highlighting quotes in order to make comparisons among participants. This also allowed me to rearrange or recategorize under newly emergent themes (Rabiee, 2004). The data was inspected for the reoccurrence of words, extensiveness of comments, repeated iterations of expressed emotions and overarching ideas (Krueger, 1994).

\section{Validity}

To ensure that the analysis of the research data was an accurate representation of the study's findings, measures of qualitative validity were employed in this study. Validity in qualitative research confirms the soundness of the research in relation to the application and appropriateness of the research methods themselves and the trustworthiness of the findings (Noble \& Smith, 2015). Additionally, implementing validity strategies ensures that the research is credible (Creswell \& Miller, 2000). Credibility is confidence in how well data collection and analysis processes address the 
focus of the study such as (a) the purposeful selection of participants who have experiences relevant to the phenomenon under investigation, (b) selecting and maintaining the integrity of 'meaning units' (meaning units are portions of textual data from which meaning is extracted in data analysis without diluting meaning), and (c) identifying categories and themes that cover the data so that the researcher can make judgements about similarities within and differences between categories (Graneheim \& Lundman, 2004). Strategies used in qualitative research to ensure validity and credibility are member checking, triangulation, thick descriptions, peer reviews, and external audits (Creswell \& Miller, 2000).

Triangulation and member checking were used as strategies of validity for the current study. Triangulation is combining two or more data sources to bolster the confidence of the research data with the aim of creatively exploring phenomena, uncovering distinctive findings, contesting or merging theories, and urges the researcher to more closely examine and understand the problem under investigation (Jick, 1979). Creswell (2014) suggested that if "themes are based on converging several sources of data or perspectives from participants, then this process can be claimed as adding to the validity of the study" (p. 201). As such, I utilized semi-structured, in-depth, one-to-one interviews, and photo elicitation during focus group to triangulate the data. The benefit of triangulation is the amount of data collected and analyzed which provides a broadened scope to grasp the totality of the phenomenon (Banink, 1993).

The triangulation of semi-structured interviews and focus group discussions strengthen the validity of the research findings, particularly if the various data sources lead to similar conclusions. More importantly, the triangulation of the data should not 
provide isolated pieces of information, but there should be "a relationship between data and methodology which relate to each other in such a way as to counteract any threats to the validity of the process” (Maggs-Rapport, 2000, p. 219). Maggs-Rapport (2000) also asserted that commonalities and shared themes in the data uncover substance of participant meaning and the particularities within subcultures. For these reasons, data collection methods were selected intentionally to produce rich and descriptive narratives. The thick, rich descriptors from participant narratives strengthen the credibility of the study (Creswell \& Miller, 2000).

Credibility was also achieved through member checking. Member checking controls for the accuracy, validity, and credibility of what is recorded during participant interviews (Barbour, 2001; Byrne, 2001; Coffey \& Atkinson, 1996; Doyle, 2007; Lincoln \& Guba, 1985). Member checking is also "participant verification, informant feedback, respondent validation, applicability, external validity, and fittingness (Morse, Barrett, Mayan, Olson, \& Spiers, 2002; Rager, 2005). The role of the researcher in member checking is to rephrase or summarize the content of the participant interview and then question the participant to determine its accuracy (Creswell, 2007). Participants either confirm or disagree with the summarization as an accurate depiction of their experiences, and if accuracy is validated then credibility is established (Creswell, 2007, 2014; Lincoln \& Guba, 1985). Creswell (2014) and Lincoln and Guba (1985) also noted that member checking can occur near the end of the study after the data has been analyzed, allowing the participants to authenticate the findings and provide feedback. Therefore, in the current study, member checking occurred after data was reported and the findings summarized. 
One of the primary purposes of implementing validity strategies is to establish dependability (Graneheim \& Lundman, 2004). Dependability takes into account “factors of instability and factors of phenomenal or design induced changes" and the degree to which either data may change over time or modifications to the data analysis process initiated by the researcher (Lincoln \& Guba, 1985, p. 299). Issues influencing dependability are minimized through "overlapping methods”, which mirrors triangulating, as well as executing data processes with fidelity (Shenton, 2004, p. 71). In this study, dependability is achieved through the overlap of semi-structured interviews and focus groups as sources for data collection. Furthermore, Shenton (2004) explained that the veracity of the data processes enables future researchers to successfully replicate the study.

To further assess the trustworthiness of data processes, the researcher may seek to explore the transferability of the findings. Transferability refers to the degree to which findings are transferable to other groups or contexts (Polit \& Hungler, 1999). The reader of the findings makes the decision on its transferability based on the researcher providing clear and precise information regarding cultural context, participant characteristics, and data collection and analysis processes. The descriptive presentation of findings lends itself to enhanced transferability (Graneheim \& Lundman, 2004). While this phenomenological study explored the lived experiences of gifted African American males at a designated site, it is anticipated that the findings of this study may be applicable to gifted African American males experiencing a gifted program under a similar cultural, social, and educational context. 
The credibility, dependability, and transferability in qualitative research provides measures of confirming its truth value. Truth value establishes the degree of confidence in the findings to be true based on the research design, participants, and the context of the study (Lincoln \& Guba, 1985). In the current study, validity strategies were employed to maintain the soundness of the data collection and analyses processes. The strategies also intended to preserve the most accurate and descriptive representation of the feelings and experiences of gifted African American males.

\section{Implications and Ethical Considerations}

I designed this study to uncover the phenomena associated with the experiences of gifted African American males and how their assessment of self-efficacy influences their academic persistence in a gifted program. Kamins and Dweck (1999) established that students who believe they are intelligent and have the capacity to do well in school are more likely to be persistent and more likely to persist than other students. In this vein, Black males with academic self-confidence believe that they are competent or excellent students.

I intend for my phenomenological study to open the door to collaborative efforts between school districts and individual schools (teachers and administrators) as the first step in initiating substantial social, curricular, and policy changes for gifted students of color. The convergence of gifted program identification and retention practices void of cultural bias, culturally competent instructional strategies, targeted professional development, and regard for external barriers to student success, assist in ensuring that the education system is not short-changing gifted African American males. African American males should be seen and treated as equal and capable contributors in the gifted 
classroom in order for them to operate at their maximum level of giftedness. The educational narrative of gifted African American males will only change when practical research findings became a roadmap for policy makers and practitioners. Practitioners hold the key to bringing about an upswing in the identification and retention of gifted African American males in gifted programs. The findings of my research exposed crucial elements of the lived experiences of gifted African American males in gifted programs that have the potential to change the trajectory of gifted education among this population of students and ultimately alter educator attitudes and approaches to teaching gifted African American males.

Protecting participants in a research study, particularly vulnerable subjects, calls for compliance with a rigid set of protocols and ethical considerations. Therefore, parental consent and subject assent forms are explicit in describing the scope of the study and the rights of the participants. It is my responsibility to ensure that the management of the data received from subjects is not compromised. Although an intimate view of the lived experiences of gifted African American males in a gifted program exposed harsh truths, disclosure did not exploit or pose threat to the participants. Sensitive issues are confronted when researching marginalized groups, such as gifted African American males, however the stories behind national statistics are worthy of exploration and explanation. As a researcher, it is difficult to approach research from a completely neutral position because of my personal history and the experiences I bring to the research process. Those same perceived limitations fueled my passion to pursue research on this topic. In full acknowledgement of the background I bring to this study, it is my 
ethical responsibility to tell the stories of gifted African American males with clarity and accuracy.

\section{Researcher's Positionality}

As the researcher, I first identified a problem to be examined and posed thoughtful research questions that address the need to uncover a phenomenon with the intent of advancing the field of study to which the topic under investigated is associated. Second, I am charged with protecting the interests of the study's participants by using protective research practices in data collection and analysis that are an unbiased and an accurate portrayal of their lived experiences. Third, it is my obligation to present the interpretations, meaning, and future implications through which the participants are empowered, thus enriching the field of study to meet the identified needs of the population under investigation. In the current study on the lived experiences of gifted African American males, I present a credible and reliable depiction of their experiences and elevate the voices of gifted African American males to be heard by educators and policymakers who have both direct and indirect influence over the quality of their education in a gifted program and educational experience in general.

As an African American female administrator in my $22^{\text {nd }}$ year of education, I bring a wealth of knowledge to the research process. In my current role, I am responsible for professional development, scheduling, grading, safety, building and grounds and transportation oversight. I am also employed at the site where the study was conducted. Based on my role of authority, previous relationships, or potential interactions with participants, every effort was made to demonstrate neutrality during the research period. Patton (2002) stated that the "quality of qualitative data depends to a great extent on the 
methodological skill, sensitivity, and integrity of the researcher” (p. 5). Although, I exercised full integrity throughout the data analysis process, my cultural experiences and background, passion, and the conviction I bring to the research process cannot be completely nullified. While these characteristics might be perceived as hindrances to the validity of the research, these elements comprise the very tenets that ground me closely to fulfilling the research process with respect for the protocols designed to produce valid and uncorrupted research findings.

\section{Limitations}

The current study exploring the lived experiences of gifted African American males and the manner in which their sense of self efficacy influences their persistence in a gifted program was not without limitations. Three limitations of the study have been identified. The first limitation is my employment at the site where the research was conducted and the administrative position of authority I hold. My position at the research site could have influenced the manner in which the participants viewed me in the role of researcher and their potential inability to negate feeling unimpeded in sharing their experiences. Although interpretive phenomenology acknowledges the conceptions the researcher brings to the research process, strict compliance with research protocols restricted personal bias to elevate the voices and experiences of the participants above my own (Smith \& Osborn, 2007).

The second limitation is that the study is unique to a marginalized population. The focus of the study and its findings are relevant to gifted African American males exclusively. It is further limited to gifted African American males in middle school, specifically grades six through eight. The findings will provide insight about this group of 
participants, but information extracted from this study may be transferred to African American males outside of the studied grade level. Incorporating African American males in the gifted talent pool in the primary grades might provide a more comprehensive narrative on the progressive impact of identification and retention practices in the recruitment of African American males to gifted programs. Examining early identification and participation trends could provide insightful information about the lived experiences of gifted African American males and inform practices designed to improve the quality of their educational experiences upon matriculation from the primary grades to middle school. Due to the focus of this study, other races and genders will not be included, therefore the findings may not relate to females or other ethnic demographics.

The third limitation is that only African American males with the appropriate CogAT score will be considered for participation in the study, even though the site may service African American males in the gifted program if they scored within one point of the designated score to be classified as gifted. The single measure used to classify students as gifted may be inadequate to measure the various ways in which students demonstrate giftedness and may limit their access to a gifted program or they may not experience the future benefits of being formally classified as gifted. 


\section{CHAPTER IV \\ FINDINGS AND ANALYSIS}

African Americans, particularly African American males, are underrepresented in gifted programs (Ford \& Whiting, 2010). Winsler, Karkhanis, Kim, and Levitt (2013) suggested that in relation to the body of knowledge on gifted African American males, more needs to be known about the factors that increase the probability of an African American male being identified as gifted, especially early in their academic career. According to Subotnik et al. (2011), if gifted children who have outstanding academic talent do not have access to participate in an intentionally developed gifted program, they are likely to perform poorly, let alone realize their full potential. As such, the reporting on the experiences of gifted African Americans in the current study not only provides an opportunity to examine the complex and multi-faceted attributes of their lived experiences, but will also serve as an academic, social, and procedural navigational tool to reroute the culture of racialized inequities in future gifted programs.

In this chapter I present research findings that emerged from the descriptive responses provided by participants during semi-structured interviews and focus group discussions, using photo elicitation to inspire a broad and reflective exploration of the experiences of gifted African American males participating in a gifted program. In this 
chapter, I discuss the interpretive phenomenological analysis (IPA) and coding process used to identify themes. I describe the themes and sub-themes that emerged from the data, incorporating substantial verbatim text to ${ }^{1}$ support emergent themes as a means of preserving the voices of the participants. I also discussed themes in the context of their lived experiences, specifically as it relates to the research questions under investigation:

1. How do gifted African American males understand and experience participation in a gifted program?

2. How do African American males perceive their participation in a gifted program as shaping their sense of self- efficacy and academic persistence?

\section{Contextual Description of the Gifted Program}

Here I frame the findings of my study through a contextual lens in which organizational structures, practices, and social behaviors shaped the experiences of African American males in the gifted program at the study site. To situate gifted African American males within the academic landscape of the gifted program further, knowledge of the student and teacher demographic data is pertinent to understanding the findings. There were 1,370 students enrolled at the ${ }^{1}$ study site, of which $37.5 \%$ were African American, 49.1\% White, $6 \%$ Hispanic, and 7.4\% were of other ethnic backgrounds (Kentucky Department of Education School Report Card, 2016). The district used a magnet program code to identify and track students in the gifted program. According to district data ${ }^{1}$, during the 2017-2018 school year, 391 students participated in the gifted program. Only 22 of the 391 students were African American, of which 11 were African

\footnotetext{
${ }^{1}$ Greater specificity in sources is not provided as this would identify the school district that served as the context for the study.
} 
American males. This means that African American males represented 3\% of students enrolled and participating in the gifted program, which represented less than $1 \%$ of the total student population. The issue of disproportionality among gifted African Americans in the gifted program, and gifted African American males in particular, is similarly reflected in the ethnic disparity among teaching staff.

For example, White teachers accounted for $94 \%$ of the teaching staff at the study site, whereas African American teachers represented 6\% or the equivalent of 4 teachers out of 69. (Kentucky Department of Education School Report Card, 2016). At the time when I conducted the current study, there was one African American teacher assigned to and teaching on a gifted program team (see Footnote 1). As such, the disproportionality among African American males participating in a gifted program and the inverse demographic characteristics of the teacher population is cogent to unpacking how gifted African American males make sense of their lived experiences in a gifted program.

The restrictive criteria used to identify students as gifted further fuels racial and social dissonance experienced by African American males in a gifted program. Students wanting to apply to the gifted program at the study site must have scored a 24 on the CogAT. In addition, the study site required students to have a cumulative grade point average of 3.2, score at the Apprentice or higher performance level on the state assessment in reading and mathematics, and have what school administrators determined to be good attendance and discipline history. Each of the criterion was weighted and given a numeric value. The sum derived from totaling the numeric value served to rank order applicants to the gifted program. Applicants ranking above the cut-off score were accepted. The number of vacancies in the program served as a cut-off for the upcoming 
year. The gifted program application process did not take into consideration teacher recommendations, artistic artifacts, evidence of leadership, writing samples, nor auditions. The criteria used for the identification of giftedness scarcely aligned with federal indicators used to identify giftedness, which include gifted indicators beyond extraordinary academic ability, such as creative, artistic, or leadership capacity (Javits Act 1997, S. 303).

\section{Summary of IPA Process}

The interpretive phenomenological analysis (IPA) process requires the researcher to engage in a sustained interpretive relationship with the data by capturing contextual complexities and the manner in which respondents make meaning of their mental and social world (Smith \& Osborn, 2007). This process involves close reading, coding, categorizing, and finalizing themes that frame the meanings immanent to the participant's experiences. As such, in my initial coding I conducted multiple close reads of the participant's semi-structured interview transcripts. The purpose of the transcript close read was to reacquaint and familiarize myself with the participant responses; intentionally not making any notations during the first read. After reading through the transcripts several times, I annotated interesting or significant content in the right margin either summarizing, paraphrasing, or using singular words to make associations, connections, and preliminary interpretations. Additionally, I annotated text when I encountered similarities, contradictions, and reverberations among and between participant responses. During the final close read, I highlighted and underscored words and phrases representative of the emerging themes. I followed the same process in my review of the focus group discussion transcripts and photo elicitation free list data. 
Following the initial coding, semi-structured interview transcripts and focus group discussion transcripts were coded using in vivo and focused coding. In vivo codes are used in identifying and explaining behaviors or processes of participants, while preserving the voice, views, and actions of the participants in the code terminology itself (Charmaz, 2014; Strauss, 1987). Focused coding organically follows in vivo coding and is used to identify significant and frequently occurring codes to extract the most salient categories in the data and requires the researcher to make analytical decisions about which codes are thematic and the most reflective of participant experiences (Charmaz, 2014). Codes from the in vivo coding process were grouped from every participant for each of the questions based on common elements among the codes to create a category. Since the creation of themes "is an outcome of coding, categorization, and analytic reflection”, grouping the codes into categories allowed me to compare the grouped codes across the participant's data to assess commonalities, comparability, and transferability, to arrive at dominant themes (Saldaña, p. 198, 2016).

To examine further the scope of the themes emerging from the data, interview questions, focus group discussion questions, and text from the photo elicitation were grouped into two categories according to their congruity with each of the two research questions under investigation. The themes were then color coded on index cards using two different colors to distinguish between the themes pertinent to each research question enabling me to construct a table of themes (Smith \& Osborn, 2016). During this stage of IPA, Smith and Obsorn (2016) suggested that themes that do not connect well with the emerging thematic structure or do not provide rich evidence in the transcript, may be excluded. Therefore, I grouped the theme cards to observe the frequency with which the 
theme occurred, to establish subordinate themes, and to identify connections or

overlapping themes across research questions, identifying what to move, condense, or

eliminate.

The interpretive phenomenological analytic process provided a dynamic approach to excavating the research data and analyzing the personal experiences and perceptions of gifted African American males. Using in vivo and focused coding specifically in the IPA process, preserved the voices of the participants throughout the coding process. The in vivo and focused codes for the semi-structured interviews were recorded on an electronic coding template (see Figure 1).

\begin{tabular}{|c|c|c|c|c|c|}
\hline $\begin{array}{c}\text { Question in } \\
\text { Statement Form }\end{array}$ & $\begin{array}{c}\text { Coding I } \\
\text { (In Vivo - } \\
\text { Participant voice) }\end{array}$ & $\begin{array}{c}\text { Coding } 2 \\
\text { (Focused Coding - } \\
\text { Theme development) }\end{array}$ & $\begin{array}{c}\text { Code Development } \\
\text { for Categorical } \\
\text { Comparison } \\
\text { (Categories \& } \\
\text { Subcategories) }\end{array}$ & $\begin{array}{l}\text { Supporting Evidence } \\
\text { (Participant Number, } \\
\text { Transcript Page, \& } \\
\text { +/- for Excellent Case } \\
\text { Examples) }\end{array}$ & $\begin{array}{c}\text { Illustrative } \\
\text { Comments } \\
\text { (longer snippets) }\end{array}$ \\
\hline $\begin{array}{l}\text { \#4 I feel accepted } \\
\text { when...I don't feel } \\
\text { accepted when. }\end{array}$ & $\begin{array}{l}\text { P1-I am accepted when } \\
\text { people include me in } \\
\text { their work. I am not } \\
\text { accepted when nobody } \\
\text { listens. } \\
\text { P2-I am accepted when I } \\
\text { am treated the same way } \\
\text { as everybody else and } \\
\text { when I'm on the same } \\
\text { level as everybody else } \\
\text { socially. I am not } \\
\text { accepted when someone } \\
\text { else is pushed but I don't } \\
\text { feel like I'm pushed. } \\
\text { P3-I feel accepted when } \\
\text { people ask me for help. I } \\
\text { don't feel accepted when } \\
\text { people say I'm wrong. } \\
\text { (As it relates to school } \\
\text { work; feeling of } \\
\text { embarrassment). } \\
\text { P4-I am accepted when I } \\
\text { am respected, but I'm not } \\
\text { accepted when I'm not } \\
\text { included; when you can't } \\
\text { share similarities. }\end{array}$ & $\begin{array}{l}\text { Category: Sense of } \\
\quad \text { Belonging } \\
\text { P1-Accepted when people } \\
\text { include me in their work; } \\
\text { Being Treated Equally } \\
\text { P2- When treated the same } \\
\text { way as everybody else; } \\
\text { P2-On the same level as } \\
\text { everybody else socially } \\
\text { P3-Accepted when people } \\
\text { ask me for help; } \\
\text { Valuing Peer Relationships } \\
\text { P4-Accepted when reapected } \\
\text { Looking for Friendship } \\
\text { P6-Accepted based on } \\
\text { whether people like me or } \\
\text { dislike me or being mean } \\
\text { like not talking to me; } \\
\text { P8- Finds acceptance } \\
\text { through humor } \\
\text { P10-Accepted where it's } \\
\text { not a toxic relationship } \\
\text { P10-Everybody cares } \\
\text { about each other } \\
\text { Category: Exclusion }\end{array}$ & $\begin{array}{l}\begin{array}{l}\text { Category: Sense of } \\
\text { Belonging }\end{array} \\
\text { Valuing Peer } \\
\text { Relationships } \\
\text { Sharing Similarities } \\
\text { Looking for } \\
\text { Friendship } \\
\text { Category: Exclusion } \\
\text { Being Treated } \\
\text { Equally } \\
\text { Being Invisible } \\
\text { Undermining } \\
\text { Intelligence }\end{array}$ & $\begin{array}{c}\text { Participants } \# 1-4, \text { P. } 3 \\
+\end{array}$ & $\begin{array}{l}\text { P2- "If a teacher } \\
\text { wanted them to work } \\
\text { harder, but didn't } \\
\text { really care for me as } \\
\text { much, and dian't } \\
\text { want me to work as } \\
\text { hard, then that } \\
\text { makes me feel like } \\
\text { I'm not accepted" } \\
\text { (p.l) }\end{array}$ \\
\hline
\end{tabular}

Figure 1. Coding template used for semi-structured interviews. 
As an example, in question four, I asked the participants, "What makes you feel accepted or unaccepted in this group?” The in vivo code captured the verbatim text of the first four participants as recorded in the coding tool. I derived the focused codes from the most significant codes extracted from the participant responses. The codes developed the most conspicuous categories and subcategories. Next, I compared the categories and subcategories in order to identify dominant themes emerging from the data. The last column of the coding tool contained an illustrative comment from the participants which supported the essence of the emergent theme.

In vivo codes also provide “imagery, symbols, and metaphors for rich category development, plus evocative content for arts-based interpretations” (Saldaña, 2016, p. 109). Therefore, in vivo coding was also apt for coding the photo elicitation data in the current study (See Figure 2). 


\begin{tabular}{|c|c|c|c|c|c|}
\hline $\begin{array}{c}\text { Question in Statement } \\
\text { Form }\end{array}$ & $\begin{array}{c}\text { Coding } 1 \\
\text { (In Vivo - } \\
\text { Participant voice) }\end{array}$ & $\begin{array}{c}\text { Coding } 2 \\
\text { (Focused Coding - } \\
\text { Theme development) }\end{array}$ & $\begin{array}{c}\text { Code Development } \\
\text { for Categorical } \\
\text { Comparison } \\
\text { (Categories \& } \\
\text { Subcategories) }\end{array}$ & $\begin{array}{c}\text { Supporting Evidence } \\
\text { (Participant Number, } \\
\text { Transcript Page, \& } \\
+/ \text { - for Excellent Case } \\
\text { Examples) }\end{array}$ & $\begin{array}{c}\text { Illustrative } \\
\text { Comments } \\
\text { (longer snippets) }\end{array}$ \\
\hline IMAGE 2 & $\begin{array}{l}\text { FGl- } \\
\text { He's trying to participate in } \\
\text { the discussion } \\
\text { He's cooperating } \\
\text { The only African American } \\
\text { male } \\
\text { The only one in the back } \\
\text { He's probably lonely } \\
\text { He's trying to get his } \\
\text { answer across } \\
\text { He's trying to be noticed, } \\
\text { but the teacher is not } \\
\text { calling on him } \\
\text { It's like he's been waiting a } \\
\text { while } \\
\text { The teacher is not focused } \\
\text { on him }\end{array}$ & $\begin{array}{l}\text { Category: } \\
\text { Invisibility } \\
\text { The only African } \\
\text { American male } \\
\text { The only one in the back } \\
\text { He's probably lonely } \\
\text { He's trying to be noticed, } \\
\text { but the teacher is not } \\
\text { calling on him } \\
\text { It's like he's been waiting } \\
\text { a while, } \\
\text { The teacher is not focused } \\
\text { on him } \\
\text { Category: } \\
\text { Acceptance } \\
\text { He's trying to participate } \\
\text { in the discussion } \\
\text { He's cooperating } \\
\text { He's trying to get his } \\
\text { answer across, }\end{array}$ & $\begin{array}{l}\text { Category: Invisibility } \\
\text { Invisible } \\
\text { Isolation } \\
\text { Loneliness } \\
\text { Faceless } \\
\\
\text { Category: } \\
\text { Acceptance } \\
\text { Validation } \\
\text { Fitting In } \\
\text { Belonging } \\
\text { Relationship } \\
\text { (Teacher) }\end{array}$ & FG1, p.2-5 & $\begin{array}{l}\text { FG1- } \\
\text { Chris-Image 2- "I feel } \\
\text { like I'm one of the } \\
\text { kids raising their hand } \\
\text { for a long } \\
\text { time...waiting to share } \\
\text { their view or answer." } \\
\text { Chris-Image 2- "All } \\
\text { the Black kids in my } \\
\text { class are separated in } \\
\text { each corner almost." }\end{array}$ \\
\hline
\end{tabular}

Figure 2. In vivo and focused coding for focus group discussion and photo elicitation.

Following the same process for semi-structured interviews, during the focus group discussion utilizing photo elicitation, the participant responses were elicited from Image 2 (see Appendix E, Photo Elicitation, Image 2). Saldaña (2016) concluded that the coding of the actual words of marginalized adolescents deepens the researchers' understanding of their social, cultural, and world perspectives, making In Vivo, appropriate for coding textual data of middle school adolescents. Therefore, using Saldaña’s (2016) recommendation for utilizing the in vivo coding process, each line or group of lines from every participant were assigned a code for each interview question, focus group discussion question, and text from the photo elicitation free lists. 
This process guided me in making sense of the experiential understandings of the participants and exploring the ways in which they created meaning from their personal actions and interactions within the contextual framework of being an African American male in a gifted program. The dual interpretation inherent to IPA not only divulges the ways in which participants make meaning of their world, but also influences the manner in which I as the researcher decodes and interprets that meaning. In other words, I attempted to understand what an experience is like from the participant's perspective.

The findings and analysis of the participant's responses revealed emergent themes unique to the phenomena under investigation. The coding process specifically, provided a systematic technique for classifying and categorizing the themes. The process of codifying allows for the separation, clustering, reorganization, categorization, and assembly of data in order to secure its meaning and to develop an explanatory narrative that presents its significance to the participants (Grbich, 2013). Therefore, the themes that emerged from the experiences of gifted African American males during the coding process unveiled the multidimensionality of their lived experiences.

\section{Participant Profiles}

Participant profiles provide descriptions about the participants themselves and their educational background as it relates to participation in a gifted program. Of the 11 prospective research study participants invited to participate in the study, the parents of 10 granted consent to participate in the study. In addition to the letter of interest sent to prospective participants, I made contact via phone to the parent of the eleventh participant. However, the parent indicated that prior commitments would not allow him 
to participate. I gave the participants pseudonyms to preserve confidentiality. Table 1 shows the grade level and years of participation in a gifted program for each participant.

Table 1

Participant Profile of grade level and years of participation in a gifted program

\begin{tabular}{clc}
\hline $\begin{array}{c}\text { Participant Name } \\
\text { (Pseudonym) }\end{array}$ & Grade Level & $\begin{array}{c}\text { Years Participating in a } \\
\text { Gifted Program }\end{array}$ \\
\hline Felix & $6^{\text {th }}$ Grade & 1 \\
Chris & $7^{\text {th }}$ Grade & 2 \\
Philip & $6^{\text {th }}$ Grade & 1 \\
Allen & $7^{\text {th }}$ Grade & 2 \\
Benjamin & $8^{\text {th }}$ Grade & 2 \\
Tony & $7^{\text {th }}$ Grade & 2 \\
Amari & $7^{\text {th }}$ Grade & 1 \\
Lee & $8^{\text {th }}$ Grade & 3 \\
Curtis & $8^{\text {th }}$ Grade & 3 \\
Danny & $7^{\text {th }}$ Grade & 2 \\
\hline
\end{tabular}

Felix. Felix was born in the United States; however, his parents are from Somalia and Somali is the dominant language spoken in his home. He described himself as an introvert, but expressed a desire to be more outgoing and engage in athletic activities. Felix was soft-spoken, his shoulders sunken, head held low, and he made minimal eye contact during his interview, yet he was notably more demonstrative among peers of his age during the focus group discussion. He excelled academically, but indicated that although he engaged in many activities outside of the classroom, he did not do well at any of them in the way he excelled in the classroom. 
Chris. Chris was a 13-year-old seventh-grader at the time of the study. He likes to play basketball and his favorite subject is mathematics. Chris has participated in the gifted program for two years and described his academic challenges as a motivation for him to excel. He expressed modesty despite his high level of academic ability. Throughout the interview process he repeatedly conveyed not thinking of himself as above anyone else because he was gifted.

Philip. Philip was boisterous, expressive, humorous, and comfortably shared his perspectives and experiences as a gifted African American male participating in a gifted program. Similar to other 12-year-old sixth graders, Philip likes to draw, play video games, play with his friends, and sleep. Philip entered the gifted program as a sit-in. In his particular case, he scored a 24 on the CogAT, which is the qualifying score to be considered gifted, however his application to the gifted program was submitted late, therefore he technically did not receive the gifted program coding to be considered until the middle of his sixth-grade year. The district uses magnet codes to track students accepted into a gifted program. In an attempt to meet his academic needs, the school allowed him to participate in gifted classes until his application was fully processed and he received the appropriate magnet coding.

Allen. Allen enjoys baseball, football, and traveling. He enjoys going to school and meeting new people. Allen recalled his fourth-grade teacher as the one who identified his giftedness. The summer following his fifth-grade year he participated in a summer enrichment program which culminated in taking the CogAT. He scored a 24 and applied to the gifted program in order to begin participating at the start of his sixth-grade year. After taking the CogAT and earning a 24, Allen himself realized his giftedness. 
Benjamin. Benjamin is confident and not at all bashful about his giftedness or academic accomplishments. As a pre-kindergarten student, Benjamin attended some classes with first grade students. Although recommended for acceleration to skip Kindergarten, a delivery model often used with gifted students, his mother decided he would attend Kindergarten for the sake of his developmental and socio-emotional wellbeing. As a third-grader, he scored in the $99^{\text {th }}$ percentile on the state assessment. As a result, he enrolled in advanced classes during his fourth and fifth-grade years. During his fifth-grade year he scored a 24 on the CogAT and was accepted into the gifted program at the start of his sixth-grade year.

Tony. Tony is a playful and light-hearted seventh-grader. He is a member of the school's cross-country team and spends his leisure time playing the guitar, video games, and tending to his pet bearded dragon. Tony's mother is White and his father is African American. Tony qualified to participate in the study because his parents identified him as African American on his school enrollment, as opposed to selecting the ethnic option for other or two or more races. Tony has taken the CogAT three times between the third and fifth grades, and each time he met or exceeded the score of 24. Although Tony was cognizant of his giftedness, he was not aware that the CogAT specifically was the measure used to determine his placement in the gifted program. He was aware that a test of some kind determined the placement, but similar to some of his gifted African American peers, he modestly asserted that his high scores on a test did not make him smarter than his non-gifted peers, but that he was better at test-taking.

Lee. Lee described himself as a loner with few friends who enjoys playing video games and eating food. He was blunt and straightforward in the retelling of his academic 
experiences. Although he recounted his elementary school experiences as generally being good, he qualified his characterization of elementary school as being "bland, and not [providing him] a stand-out or stellar education.” Lee scored a 24 the first time he took the CogAT and scored one point higher each time he took the assessment, earning a near perfect score of 27 the last time he took the exam in elementary school.

Curtis. Curtis was a markedly eloquent and articulate eighth-grader who descriptively recounted his involvement in the gifted program with depth of insight and shared candidly about his personal experiences. Curtis lives with his older brother and mother who is a single mom. His parents divorced and his dad lives in a nearby city. Curtis' older brother did not provide an academic model for him due to his own personal struggles, but his mother provided the support and structure he needed to be successful in school. Curtis loves school and his favorite pastime is doing random math problems. He described mathematics as his go-to subject. He also enjoys geography and research. He thinks of himself as being cultured as evidenced by his enjoyment of Korean pop music.

Danny. Danny is a 13-year old seventh-grader. His mother is White and his father is African American. His participation in this study is based on his parent's ethnic selection on the school's enrollment form in which they identified his ethnicity as African American. Danny enjoys playing lacrosse, basketball, and video games. He also plays the French horn in the school band. Danny scored a 25 on the CogAT the first time he took the assessment. After touring the prospective school as a fifth grader, he decided to apply to the gifted program because he liked the instruction in the classes and because he would be able to continue playing the French horn in the school band. 


\section{Emergent Themes}

Interpretive phenomenological analysis (IPA) differs from other

phenomenological approaches in which the researcher wholly immerses oneself in participant's thick descriptions, and identifies and extricates themes, to ultimately meld themes into a meaningful and descriptive narrative that reveals the phenomenon under investigation. While IPA does include these elements, it further expands the analytic process to incorporate interpretive activity, meaning the researcher interprets the situation and uses linguistic and conceptual evidence from participant transcripts to confirm or negate their interpreted understanding (Maggs-Rapport, 2000). In addition, the IPA researcher never completely disassociates from the participant, realizing that the researcher may build upon prior knowledge and experiences to interpret and understand the emergent themes and build upon new ideas (Maggs-Rapport, 2000).

IPA distinctively utilizes a considerable number of transcript extracts as well the interpretive analysis confirmed through participant responses. Accordingly, Smith, Flowers, and Larkin (2009) suggested that a narrative exposition in IPA draws upon both the presentation of data and an interpretive valuation that gives meaning to the data. In effect, the IPA presentation of narrative data is thematically driven and aggregates "a dialogue between participant and researcher that is reflected in the interweaving of analytic commentary and raw extracts” (Smith et al., 2009, P. 110). Therefore, the phenomenological and interpretive nature of IPA is preserved in the reporting of the current study's research findings, while textually and hermeneutically grounded the meaning of the data from which emergent themes were derived (see Figure 3). 


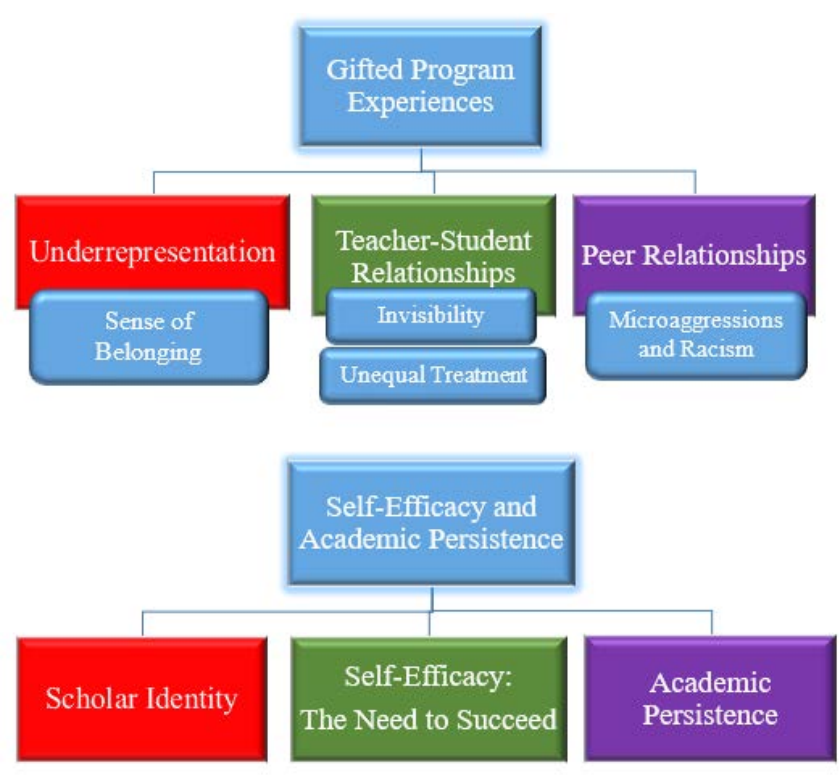

Figure 3. Emergent themes and subordinate themes.

Several themes and subordinate themes emerging from the data represented common threads interwoven across and between participant experiences. The themes offer an experiential lens from which to frame the participant experiences. The subordinate themes disaggregated the primary themes to explore the profound nuances embedded in each theme more deeply, which informed the themes themselves. While the themes and subordinate themes contain defining features, the interconnectedness of both theme and subordinate theme are necessary in constructing a narrative that credibly speaks to the experiences of the participants.

There were 39 codes from semi-structured interviews and 25 codes from the focus group discussion and photo elicitation data. From these data sources emerged six major themes that were the most salient to the experiences of the participants. In the context of the current study, six primary themes grounded the participants' experiences: (a) 
underrepresentation; (b) teacher-student relationships; (c) peer relationships; (d) scholar identity; (e) self-efficacy; and (f) academic persistence. In the context of the current study, underrepresentation referred to the low enrollment of gifted students in minoritized groups, particularly African American males. The emergent themes revealed that African American males fortunate enough to be accepted in a gifted program experienced challenges in developing teacher and peer relationship. In the context of the current study, teacher-student relationships defined the manner in which participants interpreted teacher interactions with them and their interpretation of the teacher's feelings about them, particularly with regard the way they are treated in comparison to White students. Along the same lines, peer relationships referred to the participant's need to form and maintain relationships with peers as well as their ability to moderate feelings of isolation, indifference, and racial aggression.

The additional themes emerging related to participant's exhibiting characteristics of scholar identity, specifically in shaping self-efficacious behaviors influencing academic persistence. As such, scholar identity delineated the characteristics and qualities that determined the participants approach to and success in academic pursuits. Based on the function of self-efficacy in the development of scholar identity, selfefficacy was regarded as the participant's belief and confidence in their ability to be successful in a task or collection of tasks that validated their potential and ability as a gifted student. In the context of this study, self-efficacy also incorporated the participant's compulsion to adapt personal behaviors despite obstacles encountered within the learning environment driven by their need to succeed. So too, academic persistence referred to the participant's resiliency and the perseverance needed to 
counteract and recover from the social and emotional barriers existent in a gifted program that is required to maintain high levels of academic achievement.

From the six emergent themes, four subordinate themes were extrapolated to expand upon and provide further insight into how the participants experienced and understood participation in a gifted program. The subordinate themes were: (a) sense of belonging; (b) invisibility; (c) unequal treatment; and (d) microaggressions and racism. The underrepresentation of African American males contributed to the participant's wherewithal to feel a sense of belonging in the gifted classroom. Therefore, sense of belonging in the current study was the participant's perception of acceptance and gratification in a gifted program considering the cultural, social, and racially discriminatory practices that do not support the their individual and collective need to be accepted among other gifted peers.

Invisibility and unequal treatment were themes emerging for the context of teacher and peer relationships. The emergent theme invisibility referred to the participant's feelings of being unaccepted, overlooked, or devalued by teachers and peers in relation to the perceived and observed degree of acceptance experienced by nonminoritized students. Stated bluntly, unequal treatment is the participant's perception of prejudicial and unfair treatment enacted differentially by the teacher compared to nonminoritized students. Based on the responses of participants and corroborated by scholarly literature, microaggressions and racism were defined as direct and indirect, racially discriminatory comments made to or about the participant which called into question their academic ability or right to have access to or participate in a gifted program (Allen, Scott, \& Lewis, 2013; Nadal, Wong, Griffin; Davidoff, \& Sriken, 2014; 
Stambaugh \& Ford, 2015). In the current study, the emergent themes and subordinate themes revealed the manner in which African American males defined their scholarly identity, perceived their relationships, and maneuvered within their academic environment. The emergent themes and subordinate themes also gave clarity to the participant's personal capital which allowed them to articulate an academic counter narrative by living out self-efficacious behaviors, which intern positively influenced their academic persistence. Collectively, the themes and subordinate themes conveyed the phenomenon distinctive to this group, yet regarded the participant's individual experiences, without which their collective voices would not be heard.

\section{Experiences of Gifted African American Males}

The participants in the current study reported both positive experiences as well as the challenges they faced as an African American male participating in a gifted program. When asked about their experiences in a gifted program, the participants responded that overall their experiences were good, using terms such as pretty good, pretty well and enjoyable. However, as the discourse developed, they mutually cited instances of alienation, unacceptance, unequal treatment, and racial bias.

While the participants described the gifted program as having challenging curriculum, copious assignments, projects, and tests indicative of the academic rigor expected in a gifted program, their assessment of experiences as participants in a gifted program were primarily rooted in the way in which they perceived their relationships with teachers and peers. As such, this section will examine the underrepresentation of African American Males and the degree to which underrepresentation influences the participant's sense of belonging, teacher-student relationships, the participants' feeling of 
invisibility, and the manner in which participants manage peer relationships amidst overt and covert microaggressions and racism.

\section{Underrepresentation}

The reality of the underrepresentation of African American males in the gifted program reverberated throughout the participant's responses. They recognized the underrepresentation of students from the same ethnic background as more than a numeric disparity, but as the vehicle perpetuating a cultural disconnect in the gifted program. Furthermore, their responses alluded to the underrepresentation of African American males in the gifted program as disrupting the maturation of unbiased teacher and peer relationships and the development and the perpetuating of stereotypical narratives about the academic acumen of African American males.

To this point, Chris recalled his experience as a second grader attending a majority White school when he explained, "I looked around and noticed, I'm the only Black person in here.” He compared his second-grade experience to his middle school gifted experience, and stated, "It's kind of the same thing with GT, because maybe there will be a couple other Black girls, but I feel like, I don’t think I have any classes with another Black GT kid that's a boy. It's like I'm the only one in here.” Philip's illustration (see Figure 4) of his experiences in a gifted program, typified the thoughts on underrepresentation described by the participants. 


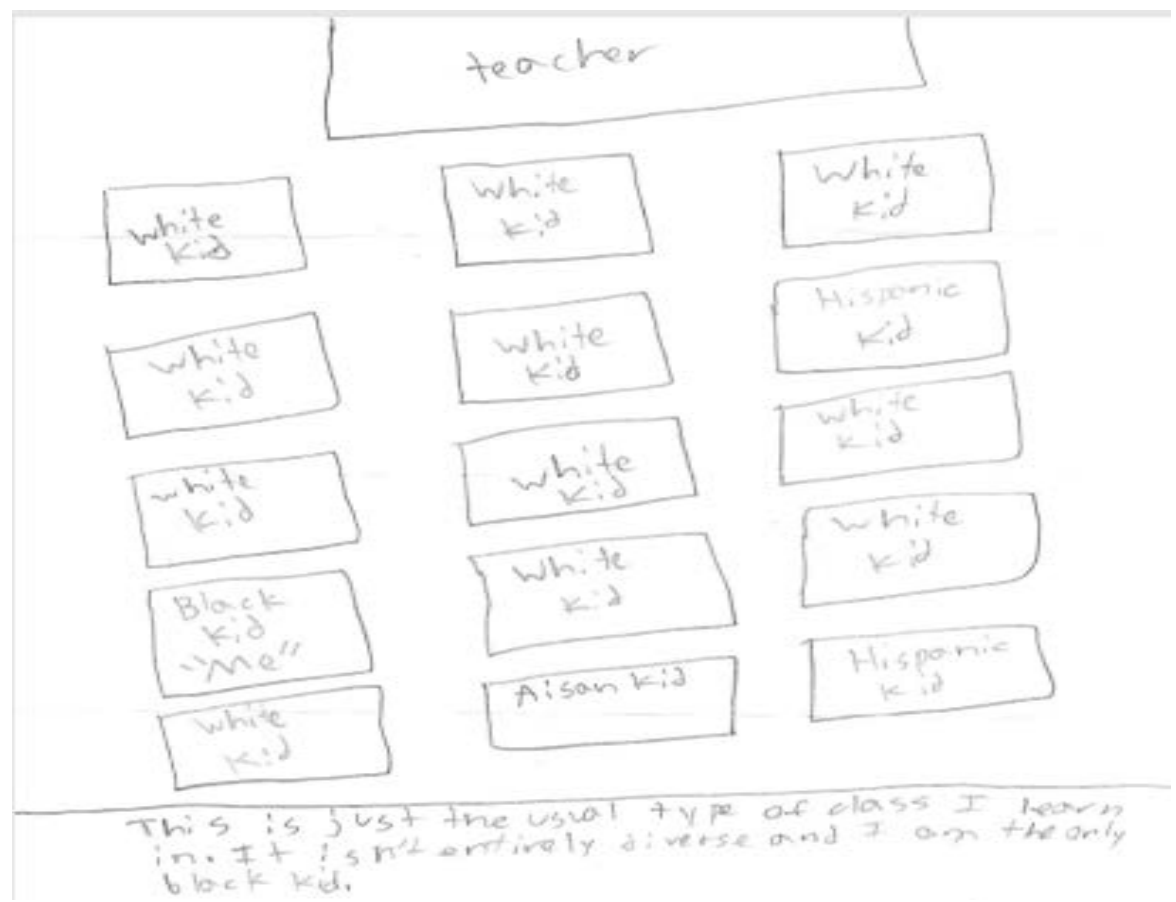

Figure 4. Participant's illustration of underrepresentation in a gifted program.

Allen shared similar thoughts when he stated, “In some of my classes, where I'm mainly like the only African American male in that class, sometimes when you can't share similarities with somebody, then that might not make you feel accepted.” Additionally, when Allen expressed his thoughts about the image he identified with least during the photo elicitation exercise, he selected Image 4 (Photo elicitation, Appendix E, Image 4), and stated that he could not identify with the image "because there's really not a lot of African American kids or teachers in the classes that I've been in.” Lee responding to the same image confirmed this when he said, "Yeah, I’d say most, majority of the classes have predominantly more Whites.” To further illustrate this point, Curtis also shared his thoughts from his experiences in the gifted class and upon his reflection would rhetorically ask himself “How come there aren’t that many African American kids 
in this program? or, Do they just not like accepting African American kids?” Curtis went on to share:

Sometimes I felt like, especially in $6^{\text {th }}$ grade, I was the only African American in most of my classes...in my social studies and science class. There was one other in my math and Language Arts class. But I feel like that's one of the things that's always kept in the back of my mind, that I didn’t like. I fit in as far as my academics, that kind of stuff, but I didn’t fit in. I just didn’t. I feel like I fit in, but I didn’t fit in.

Curtis made emphatic proclamations in his reflection of the underrepresentation of gifted African American males in gifted programs when he stated:

There are African American gifted males in this world and I feel like we shouldn't be overlooked. We should be welcomed. We should be accepted. We should have opportunities. We should have more support. I would say as a whole we are tired of being underrepresented.

Curtis' reflection on the issue of underrepresentation reflected the communally oriented attributes of African American students when he took a stance, speaking on behalf of other gifted African American males to be embraced in the gifted program (Gooden \& McMahon, 2016).

Benjamin assumed a universal responsibility for the status of the underrepresentation prevalent in the gifted program when he said, "So being one of the only Black kids in the class, you have to do, you have to do your best for all the kids that are not in there.” In contrast to the other participants, while Benjamin acknowledged that there were few African American students in his gifted classes, he appeared unfazed by 
the underrepresentation of African American males in gifted classes when he commented during his interview, “I'm not in classes with them, and that's where most of the African American kids are. I’m not really upset that there aren’t any Black kids in my class. I don’t really care who my friends are.” Divergent from his previous statements, he disassociated the role of race in the underrepresentation of African American students in the gifted program.

The participant responses captured the gamut of feelings from their experiences as it related to the underrepresentation of African American males in a gifted program. Chris’ comments about the position of African Americans in a gifted program demonstrated the intersection of underrepresentation and sense of belonging when he stated, "If you're not part of the majority race, you feel like you're out of place. So, that's just what I live with, but it's just kind of weird at the same time; sometimes when you really think about it.” To further explore Chris’ sentiments, the following section uses the participants' underrepresented voices to convey personal thoughts and feeling about their sense of belonging in a gifted program.

\section{Sense of Belonging}

In the current study, sense of belonging referred to the participant's experiences and feelings of being accepted while navigating the aversive cultural, social, and racially discriminatory practices existent in a gifted program. The participant's sense of belonging influenced how they situated their position in the gifted program and moderated their feelings of not being accepted. Specific instances regarding the degree to which the participants felt accepted in the gifted program contributed to: (a) the way they 
perceived their academic ability was validated by their teachers and peers, (b) their connectedness to non-African American students, and (c) their ability to be able to fit in with non-African American peers.

Chris explained his feeling regarding not being accepted by a teacher. He stated:

I feel accepted when I'm treated that same way as everybody else...If a teacher wanted them to work harder, but didn’t really care for me as much, and didn’t want me to work as hard, then that makes me feel like I'm not accepted, because it feels like you're really not in it, I guess.

In this scenario, Chris interpreted the teacher's expectation of him in comparison to the expectations the teacher had of non-African American students as contributing to his feeling of being unaccepted and displaced. Amari expressed a similar concern when he said, “I just don’t feel like I fit in with some of the other people that are in the gifted and talented program. I find that some activities... it’s just, they like different things.”

To take another case in point, Curtis reflected on his experiences with regard to fitting in inside and outside of the gifted program when he expressed:

I feel blessed, but also feel like I can’t really fit in anywhere, because I don’t fit in with non-minorities, as far as being gifted, but I also don't fit in with my minority, because I'm not, it's hard to, you're not trying to put a stereotype around it, but most of the non-gifted, or I don't know the word, the comprehensive, they're usually the African American students. The only thing I have in common with them is my ethnicity. So, I feel like I can’t, not that I can’t get to know them, ‘cause I’ve gotten to know lot of them pretty well, but I feel like I can’t be myself around them. So, it's like a weird place that you almost have to create for 
yourself. Having to do more to fit in with everybody else whose gifted, or even fitting in with Comp kids or something, I feel like it was harder to fit in with either. You don’t really fit in with either side. So, we're like our own kinda group.

Curtis' response was the first to indicate an inability to fit in within a gifted program where the majority of the students are non-African American, yet not being able to fit in with African American students who were not in the gifted program. His experiences reflected the unique difficulties gifted African American males encounter when their giftedness, gender, and race combined makes it difficult to be included inside and out of a gifted program.

Based on the participant's responses, the influence of their sense of belonging on the social, emotional, and academic facets of their experiences in a gifted program, shaped their ability to adapt and function in the gifted learning environment. The participants' responses also revealed their inner voice conversations as a means of coping and making sense of the isolation they felt not being accepted within a learning community where acceptance is the theoretical, ideal, and expected norm for nonminoritized students. Additionally, the participant's gave insight into the routine struggle of fitting in with their same-ability counterparts. Moreover, the participants contended with what DuBois (1986) referred to as unreconciled striving; the dual reality of feeling excluded among White gifted students in a gifted program, and not being embraced by peers of their own race outside of the gifted program. 


\section{Teacher-Student Relationships}

\section{Unequal Treatment}

The participants in my study attribute their academic success in part to teachers who pushed them academically. In addition, the participants' experiences and subsequent feelings about their sense of belonging encompassed their level of engagement and perceptions of equal treatment by teachers in comparison to non-African American students. For example, Lee shared an experience from his $6^{\text {th }}$ grade year, and recalled, "Sixth grade was difficult for me because I did not enjoy my math teacher that much.” His engagement with his teacher toward building rapport was limited because of his feelings about the teacher. From a different perspective, Chris shared "I feel like teachers care for me and they want me to be at the best of my ability.” Similarly, Curtis reflected on his relationships with teachers and stated, "Ever since Kindergarten, my teachers have always pushed me, so I feel like throughout school I have been pushed like I need to be.”

Although the participants felt they were pushed academically, their viewpoints about their relationships with teachers revealed instances of unequal treatment in comparison to their White counterparts. Phillip remembered an incident in which he interpreted the teacher's response to be racially biased and unfair. Phillip shared:

We'd all be like dancing or something in the hallway, like while we're in line for the bathroom, we'd just be like doing something silly, and the teachers would call me out and they'd see my friends doing it, and then they just wouldn’t say 
anything. They'd call me out and give me some kind of consequence and they'd just give my White friends a slap on the wrist.

When asked why he thought the teacher responded in that manner, Philip said:

I think it's because they think, oh well, I should just trust, I should just trust my ethnicity of students, anybody else is just wrong, well because some people see the average Black person as somebody who would take my wallet, or who would hurt me really bad, when that's not true. You get on the Black kid because he's doing something wrong, but you let the White kids get off when they're also doing the same thing (see Figure 5).

The encounter left an indelible impression of differential treatment and bias based on race founded in the vilification of African American males as presented and reported in print and visual media.

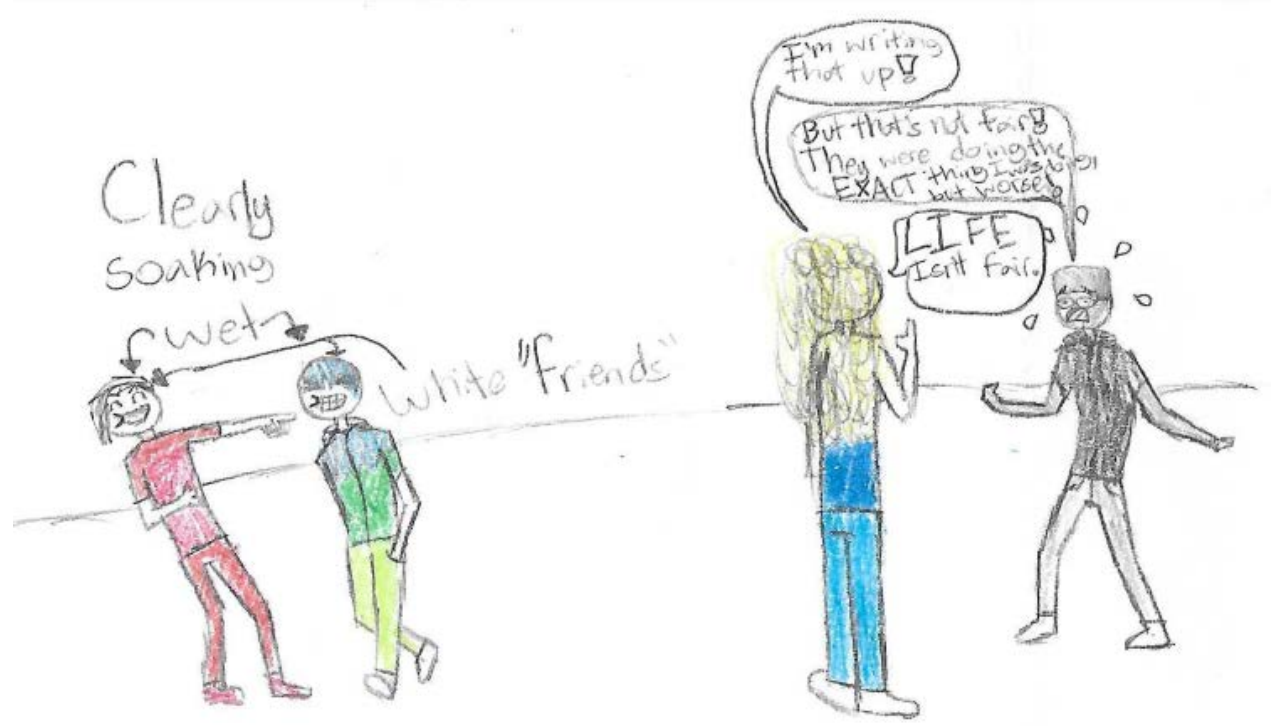

Figure 5. Teacher's treatment perceived to be racially discriminatory and unfair. 
Interpreting the meaning behind teacher-student interactions presented challenges for the participants, particularly in reconciling the dichotomy of being both accepted and isolated within the same educational setting. For instance, Curtis stated:

I love school. School, I feel like it's been the highlight of my life. Ever since kindergarten my teachers always pushed me. So, throughout school, I’ve been pushed like I need to be. I feel like being in a gifted program, it hasn't been hard, it hasn't been difficult. It's been something that's pushed me, but I feel like it's the one thing that's kept my mind focused on education. I feel like if I just would've went to any school, not AP, gifted, whatever, I feel like I wouldn’t have kept good grades, maybe I wouldn't have been the person I am, I don't know. But I feel like my experiences have been, I don't know how to phrase it. Just my experience overall, it's been a good experience.

In light of Curtis' evaluation of the supportive role teachers have played in his life, he also offered a contrasting perspective regarding his interaction with teachers. When asked if he had ever experienced discrimination while participating in a gifted class, he said "the source of the discrimination is sometimes the teachers. They'll look at me and they'll talk to one person another way and they'll look at me and start having that baby talk kind of conversation, like they're talking down to me.”

In another instance, Curtis recalled an interaction with a teacher concerning a class project, he explained:

I had a project that she said she would not accept late. I emailed it to her and she didn’t get my email apparently, so I told her about it the next day, and she said I didn’t email it to her. I was trying to reason with her, so whenever the parent- 
teacher conference came around, she told my mom I had anger issues when I was just trying to have a simple conversation with her. So, I feel like sometimes, not just African Americans, maybe like the minority students, they [teachers] like to exaggerate what some of them do. And for me, I feel like that's what causes a lot of minority students to act out, because their teachers don't give them any credit when they're actually trying. It's kind of like the small things that add up. As it relates to building and establishing trust in the teacher-student relationship, Curtis also shared:

Sometimes the teachers, they'll ask, who wants to do this or who wants to do that, and I feel like they don't trust me. For example, who wants to come up to my class early and help me hang stuff up? or Who wants to help me sort out papers? or just small things. And I’m just, I don’t know, I’m just like, no use trying, they're not going to choose me.

Amari echoed similar sentiments about trusting relationships with teachers evoked from Image 2 (Photo elicitation, Appendix E, Image 2) and stated, “They don’t trust us or want to be by us. The teachers don't want us to be close [to each other or to them] in the classroom.”

The experiences illustrate the participant's need to trust the teacher and be trusted by them, which they found to be an essential element to building a meaningful relationship as it related to increasing their sense of belonging and feeling accepted.

When discussing teacher-student relationships, the participants also expressed their thoughts on the role of the teacher's race in relation to their experiences in a gifted program. 
Tony's thoughts elicited from Image 4 (Photo elicitation, Appendix E, Image 4) led him to say "I've had one [African American teacher] and that was my best year in school; my favorite year.” In response to the same image, Curtis shared:

My greatest experience was probably when I had an African American teacher in the third grade, 'cause I felt like she was almost like a mom in the classroom, because she got to know me well. I was comfortable around her and she made me feel included. I wasn’t overlooked in her class.

Uncertain about ever having an African American teacher, Danny said, "I mean, most of my teachers have been Caucasian. I don't know if I've ever had an African American teacher. I think there's a good amount more of Caucasian teachers than there is African American.” Despite, not ever having an African American teacher, Danny expressed: My best experience as being an African American male in GT was when I came in $7^{\text {th }}$ grade, the first day I saw the new teacher [African American]. I felt confident because I think that she's showing that African Americans can do just as much as Caucasian people can.

Based on the participant's responses, the idea of having an African American teacher or knowing that other gifted African Americans had access to an African American teacher in a gifted program, had the potential to positively affect the experiences of gifted African Americans in general. To use a specific example, Amari said "I feel like they would understand me more. I feel like I could probably be more open to them.” The participant responses indicated their consciousness of their potential difference in treatment when being taught by a White teacher versus an African American teacher. The participants perceived the treatment of African American teachers to be relatively 
more nurturing and recognized that having an African American teacher meant they were more likely to understand the experience of African American adolescent males. Furthermore, their responses conveyed that the presence of an African American teacher in a gifted program instilled a sense of pride and was an indicator of hope for gifted students of color enrolled in a gifted program.

The examples shared through the experiences of gifted African American males speak to the complex dynamics of their academic and social experiences in informing how their teacher-student relationships affected them based upon teacher behaviors and teacher race. Furthermore, teacher-student relationships influenced the extent to which participants felt invisible in the academic environment, thereby having implications for their sense of belonging in a minoritized group. In addition to the participant accounts of experiencing inequities in the gifted program from the lens of their relationships with teachers, they also disclosed how feelings of invisibility in the gifted classroom dismantled their sense of belonging and restricted or inhibited the development of their relationship with their teacher.

\section{Invisibility}

In the context of the current student, invisibility referred to the participants' feeling overlooked, unaccepted, or devalued in relation to the perceived and observed acceptance experienced by their non-minoritized peers. The participants shared situations in which they were conscious of feeling unrecognized as an active participant in the classroom in the way that their White peers were recognized. Moreover, the participants described instances in which they persisted, yet struggled to find a point of 
entry to be included in classroom discourse as if they could not and would not be seen by their teachers.

For instance, Chris described an instance in an English class, he was sitting in the back of the class and wanted to join in the discussion in which the students were giving characteristic of an object. Chris recalled:

There was this one that I really, really wanted to get across, but the whole time I didn’t get to do that. I think she was only about to let two or three more people go and someone finally said it, but I had my hand raised for like the past ten minutes. Yeah, I didn’t get to get my point across.

During the focus group discussion, Chris made a direct connection with Image 2 (Photo elicitation, Appendix E, Image 2) and interpreted the image in light of his experience and stated, “Like the kid wants to get a point across, but can’t because he’s in the back of the classroom. He’s been waiting for a while.” Likewise, Allen’s illustration (see Figure 6) depicted his experiences in a gifted program, confirming Chris' feeling of being invisible.
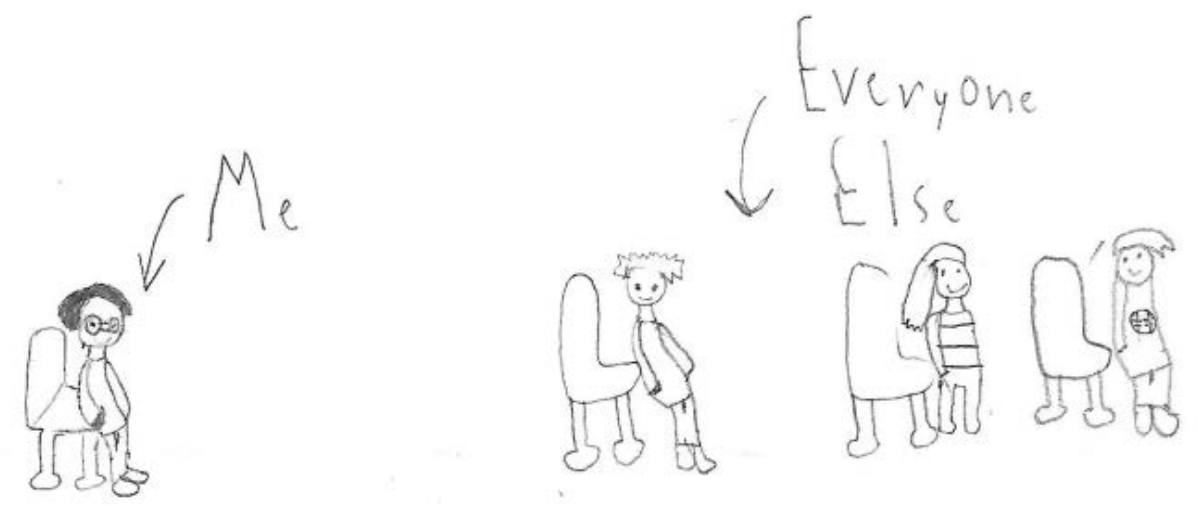

Figure 6. Participant's illustration depicting invisibility. 
To further support the notion of invisibility, Allen's illustration gives insight into how gifted African American males see themselves as a disconnected, isolated, and invisible member in a gifted classroom. The illustration provides a clear and unavoidable message about the exclusion Allen experienced and gives insight into the indelible experiences that form the social and academic narratives of African American males in a gifted program. Other participants made striking comparisons to Allen's during the focus group discussion. Lee, interpreted Image 2 (Photo elicitation, Appendix E, Image 2) through the lens of his experiences and offered to the group, "Does the teacher even see me?" Chris followed up and added, "He’s getting aggravated because he thinks the teacher doesn't see him. He might be getting used to it...yeah, one of those, oh it's this again, type things." Curtis interpreted the image to say, "I’m tired of being overlooked.”

The participant's responses with regard to feeling disregarded, exposed the tangibility in the concept of invisibility in the classroom. The participants grappled with feeling unaccepted based on how their teachers overlooked them when they attempted to participate in the same ways as their non-minoritized peers. They expressed their frustration and lassitude with feeling invisible and resigned to the neglect of the teacher as a standard behavior. The participants made connections with their feelings of invisibility based on how they believed the teacher to be accepting of them as individuals and the manner in which they demonstrated esteem for their ethnic orientation.

Specifically, the participants gauged the depth of their relationship with the teacher based on how they perceived the teacher valued their ethnicity. Meaning, they associated their sense of belonging to the teacher's intentionality and thoughtfulness in integrating culturally relevant instructional practices. The participant struggled to 
identify instances in which their ethnicity was valued in an evident or enduring manner through the implementation of the culturally relevant curriculum and class activities. When the participants were asked to describe an experience in class that made them feel their ethnicity was valued, Felix and Benjamin said, "I don’t really know a situation." Tony stated, “That's hard for me to think of one.” Lee said "Nothing comes to mind where I was a vital asset in their mental process of deciding and thinking things because of my race.” Allen and Danny referenced Black History month as the only time when they felt the teacher valued their ethnicity. As such, their responses indicated the existence of a cultural void in the instructional practices used by teachers in the gifted program. They describe their academic environment culturally sterilized and not taking into consideration the necessity and meaningfulness of establishing relationships through the knowledge of the student's cultural backgrounds in its relevance to maintaining a supportive culture for learning for African American males.

\section{Peer Relationships}

Participant responses demonstrated the significance of peer relationships to feeling connected to and accepted within the gifted program. They referred to their need to make friends and have friends in order to feel social integrated into the gifted community. The participants felt they were able to make friends, however considering those friendships, they were also conscious of how their peer relationships either made them feel accepted or unaccepted in the gifted program. They shared specific instances in which their engagement with White peers in the gifted program presented challenges to establishing and maintaining authentic relationships. Their responses reflected how 
they neutralized and made sense of the microaggressions they encountered, finding it necessary to dismiss or adapt to the exclusionary behaviors of their White peers.

As an example, Felix recalled a time when his class was working in collaborative groups.

He stated:

What makes me feel not accepted is like if nobody listens. I feel accepted when people include me in their work. We had to pick groups and nobody would pick a group with me. I would just work alone sometimes. Sometimes my friends wouldn't include anybody of my race.

Felix excused the culpability of his peers by saying, "But I don't think they meant it like that”. Despite being excluded by his classmates and having the suspicion that the exclusion was based on his race, he still referred to them as friends. Allen corroborated Felix's response when he said, “If I try to be included and I'm not included, then it makes me feel not accepted.” Conversely, Chris found his peer group to be supportive when he said, “I feel like I’m surrounded by kids that want me to be at the best of my ability. It's like we all push each other." He further explained "So even though there are kids that can be, kind of like, maybe they just don't have necessarily, like a very educated mind towards race or religion or something, but there's a lot more kids that do understand than who don't.” In an attempt to make sense of the role of race in his peer relationships, Chris continued the discussion and said, "Some kids just assume that because I’m Black, I should be like this or that. Like I could have just the same amount of characteristics as a White person.” He also expressed a necessity to help his White peers understand the experiences of African Americans as a means of building relationships when he 
commented, "I can help other people to understand what it's like to be a Black person or something, when they don’t seem to get it because they don’t live my life.”

The participants shared that making friends in a gifted program largely

determined the race of the friends they had. For instance, Benjamin shared that his sister observed that he did not have any African American friends. Benjamin was the only participant who indicated that his race had no bearing on his ability to build peer relationships. He explained, “I feel accepted by everyone. Everyone’s nice. So, if there were a group of people talking, I usually just walk up and just listen in on their conversation and act like I know exactly what's happening and stuff.” He was unaware of the distinction between open acceptance in a peer group and inserting himself in a group and considering himself as accepted. This provides evidence of an unawareness of the genuine formation of peer relationships and brings light to how African American males disregard social cues and interject themselves within groups to find acceptance.

\section{Microaggressions and Racism}

For the purpose of the current study, microaggressions are defined as direct or indirect, racially discriminatory comments made to or about the participant, which calls into question their academic ability or right to have access to or participate in a gifted program (Stambaugh \& Ford, 2015). The participants recounted instances in which comments made toward them diminished their academic ability and casted doubt on the validity of their giftedness. The participants also shared incidents of overt racism experienced in the gifted program. They provided understanding into how they managed 
the racism and how they tranquilized its potential of having deleterious effects so they could peacefully exist and function in the gifted learning environment.

For example, Chris shared, “They separate me from another person because I’m Black. I've had someone say, why are you in this GT class?”, as if to say he could not possibly be smart enough to be in a gifted class. Chris explained the perception of his White peers in thinking that all African American students were in the Comprehensive program, perceived as a substandard program in which minoritized students were significantly overrepresented. He discussed how his White peers rationalized his enrollment in a gifted program when he recalled the comment, "Okay, well the reason why you're not in Comp is because you're White.” Meaning, although they could clearly see he was African American, Chris said they meant, 'I'm not ghetto or because I don't talk the way they [Black students] do or because I don’t wear what they wear and stuff like that.”

Chris also shared an instance in which he had to defend his parent's expectation that he brings home all A’s. Chris told his peers, “If I wasn’t a straight A student, my parents would get really mad at me and stuff." He continued, "Some [White] kids don't believe me when I tell them that.” This comment supported the fact that his White counterparts who appeared supportive of him in the classroom, and had knowledge of his exceptional academic ability as a gifted student, associated low expectations and low academic performance with students of his race, which in turn applied to him specifically.

In another instance, Philip described the degrading of his intelligence in front of the class when the students were presenting their mathematical reasoning after solving a 
math problem. Some of the students were struggling with the problem and he was able to help them to understand the problem and be prepared for the whole group share-out. Philip described his being selected to share out with the group and made a minor error when he reported out the answer. Philip remembered one of the classmates saying, "I thought you had to pass the GT test to get into this class, or did someone take it for you?”. In this instance, his classmates were cognizant of his exceptional ability in math. In fact, they asked for his help, when they struggled with the problem, yet publicly embarrassed him and discounted his ability and the legitimacy of his enrollment in the gifted program.

The participants' description of microaggressions are evidentiary of the pervasive racist paradigms that persist in gifted programs. In addition to the microaggressions and microinvalidations experienced by the participants they also shared instances in which racist comments were blatantly offensive. However, due to a lack of feeling empowered and supported as a participant in the gifted program, they dismissed the offenses. By dismissing the racially offensive comments, the participants felt they could nullify the offense to avoid confrontation and avoid further exclusion among White peers.

As an example, Lee discussed a scenario in which the students were engaged in an English project highlighting the achievements of unsung African Americans. Lee commented, "I feel like people did it a bit reluctantly. In fact, someone made this joke in class where they're like, Can I do the chicken man, Colonel Saunders?” Lee explained that the student was aware that Colonel Saunders was not African American, but made the racially disparaging remark as a stereotypical association with African Americans and fried chicken. Lee added, “I just let it slide because it’s not my issue. That's not for me 
to get into.” Lee acknowledged the racist comment, but chose to dismiss it. This passive response was common in participant responses. Not because they did not absorb the perniciousness of the comments, but they perceived them as related to an insurmountable cultural norm too engrained that any immediate response would be able to undo.

Philip shared another example of racism when his White counterparts, who were a part of a team, made fun of his hairstyle. He stated:

They called me 21 Savage. It just made me really sad. I just didn’t feel accepted in a group where, I just didn’t feel accepted in a group where everyone else didn’t like me and I was trying to participate in something where we all had to work together as a team.

The participant did not know that 21 Savage was an American rap artist. However, the manner in which the rapper's stage name was used was interpreted by the participant as an insolent description. He perceived the name-calling to be rooted in the connotation of the term savage as one who is uncivil, violent and often used to describe the demeanor of a ferocious animal. Philip indicated that he did not address the comment with his peer, but continued working as a part of the team while masking his hurt.

Collectively, the participants were non-confrontational in instances of microaggression and racism in the classroom. They dismissed such comments and indicated that they felt confronting the comments would exacerbate their feelings of not being accepted in the program. The participants also indicated that they found the comments to be disruptive in their pursuit of building relationships with their White counterparts. Nonetheless, they remained confident in their academic ability and undeterred from continuing in the gifted program. 


\section{Self-Efficacy and Academic Persistence}

The participants in this study shared their perceptions and understanding of the role of self-efficacy as it related to their academic persistence as gifted African American males in a gifted program. In the context of the study, self-efficacy is the participant's disposition and level of confidence in the ability to be successful in a task or collection of tasks that validate intellectual potential and ability as a gifted student. Although the participants did not use the term self-efficacy during interviews and focus group discussions, their descriptions of perseverance aligned with the contextual description of self-efficacy for this study and were indicative of the scholarly disposition and behaviors necessary to be successful in the gifted program.

Furthermore, the participants described their approach toward the learning process as a product of the way in which they understood and demonstrated their giftedness. The participant's self-concept was tightly married to their motivation to succeed and earn recognition for their scholarship. Moreover, the participants indicated that in addition to personal drive, support systems contributed to their ability to persist academically considering the racial, social, and academic barriers experienced in the gifted program. The following sections reports the participant's experiences as it related to: (a) scholar identity; (b) self-efficacy and the need to succeed and be resilient; and (c) academic persistence systems of support contributing to academic success.

\section{Scholar Identity}

The participants in the current study exuded confidence in the way they described their academic ability. They self-validated their position in the gifted program based on satisfying the qualifications necessary for identification, admission, and participation in 
the program. The participants saw themselves as having equal intelligence as their White counterparts. One can contribute this desire for recognition as studious and scholarly to scholar identity. Whiting (2006) defined scholar identity as one in which the individual perceives himself or herself to be astute, intelligent, resourceful, and capable of excelling in academic pursuits. This definition appropriately characterizes the ethos and behaviors described by the participants.

An example characteristic of scholar identity was evident when, Chris shared, "I think I should be in here [gifted program] because I earned my way in here. I worked hard so that I could get up to this spot; so academically, I feel confident.” The participant affirmed his position in the gifted program as an earned accomplishment resulting from his academic diligence. Along the same lines, Lee discussed his academic ability and approach to learning, when he stated:

I start the year off pretty well with a schedule of what I'm going to do each day.

I'm a pretty good learn-on-the -spot person, who can just see what they're learning about, and then just learn at that moment.

In the same manner as Lee, the participants used either mental or physical organizational tools as a means to systematize the use of their learning material and the processes by which they acquire knowledge. While the participants collectively agreed that there was an abundance of challenging work in the gifted program, none of the participants indicated they felt the work was beyond their intellectual scope.

In addition to the dispositional traits associated with scholar identity, the participant's responses indicated a conscientiousness with regard to grading. Their references to grades and emphasis on making good grades was common among all of the 
participants. For instance, Benjamin said, “I feel like since I’m making all the appropriate grades, and nothing's really that hard for me, I feel like I can be successful in this program and stuff." Likewise, Lee reasoned through a situation in which he received a B in Algebra. He stated:

This one time I got a B. It was an 89.55 or something like that. It could have easily been as A, but I missed one test correction just because I had to do another thing for math. I haven’t gotten a B in social studies or science, and that's good! Similarly, Allen shared his thoughts on his feelings associated with earning good grades and explained:

Just any academic achievement like doing well on a test or doing good on any work, I see it more as an accomplishment, where others might see it as just an everyday grade. Just even small grades, I see them as really big accomplishments.

The participant's occupation with the idea of earning good grades and producing good grades provided further evidence of their comparable motivation and intelligence to that of their non-minoritized peers toward excelling in a gifted program. They divulged however, that any poor grades they received was because of a personal choice such as not studying or failing to submit assignments on time. In which case, they quickly rectified the situation to recover their academic standing.

Beyond earning good grades, the participant's attributes reflective of scholar identity established their merit to be in a gifted program. Even though they were aware of the fact that their academic performance secured their placement in the gifted program, they collectively expressed appreciation for the opportunity to participate in the program. 
In a specific example, Amari expressed thoughts on his standing in the gifted program when he stated, “I feel I’m a pretty intelligent person. I've always liked school and it's

not really ever been hard for me. I think my work ethic is pretty good. I'm proud I could say that I'm in a gifted program.” Based upon the participant's responses, their appreciation stemmed from a rudimentary understanding that there were other gifted African American students who may have been just as gifted as they were, but because the criteria by which students are identified for the gifted program is culturally exclusive, they were excluded.

As a whole, the participant's scholar identity shaped the way in which they visualized themselves as intellectuals, which is counter to the overwhelming narrative on the academic forecast of African American males. The participants embraced the idea of being studious and exhibited studious behavior in the gifted classroom. In fact, the participant's confidence in their ability to perform at the same levels as their Whites peers, served as a driving force to their academic success and in shaping their sense of self-efficacy.

\section{Self-Efficacy: The Need to Succeed}

In the context of my study, self-efficacy is the participant's internal beliefs about his level of confidence and competency to succeed and achieve the desired outcome (Bandura et al., 2001). Although the participants did not use the term self-efficacy explicitly during semi-structured interviews or focus group discussions, the experiences shared by the participants gave insight into how their sense of self-efficacy shaped their academic persistence. The participants in the current study cited instances in which their 
need to succeed was the impetus behind the certainty of their academic aptitude and determination to achieve success.

As an example, Lee discussed his resolve to demonstrate mastery in the content areas when he shared:

I keep A’s and turn in my work every day. I make sure to get all the necessary things in my brain to do well on tests. I make sure I always do well on mastering items. Those are my definite [things] I have to do good on. I make sure to do the other things too.

The participant made appropriate associations between academic behaviors, academic performance, and his realized potential to be successful. The participant expressed his desire to earn A's and demonstrated the corresponding action needed to achieve the desired outcome. When asked why this was important to him, he responded, "I have a need to achieve!"

Parallel thoughts surfaced from participant responses elicited from interpreting Image 1 (see Appendix E, Photo elicitation, Image 1). Making a personal connection to the image, Chris interpreted the image through the lens of his experiences. The images prompted Chris and he shared:

We're trying to do group work, but there are these one kids that really weren't doing any of the work and I was trying to get them to do some. Me and my friend were the backbone of the whole project and they just kind of did whatever. They would say, oh my gosh, why are you making such a big deal about it, we'll work tomorrow. Of course, they didn't. My work and my grades are too important to just be so nonchalant. 
Chris' insistence on his team working toward completion of the project, reflected his ownership of the task and the message that completion of the task conveyed about his ability to be successful. This is important to note because the participants expressed that when tasks or projects are completed and of high quality, there was an assumption that White students presumed that based on the level of quality, it most likely was not done by a student of color. Chris added his personal connection to image 1 (see Appendix E, Photo Elicitation, Image 1). When asked, what do you think the character might be thinking? He offered, "Like the work matters and he needs to achieve a goal." The statement directly reflected the sentiment of the account he shared.

The participants viewed their participation in the gifted program as the vehicle propelling them to act upon their internal beliefs regarding their level of academic competency. Additionally, the participants capitalized on their participation in the gifted program as a means of developing self-efficacious behaviors. To put it another way, they demonstrated their scholastic aptitude through accomplishing tasks successfully and persisting in the face of social and academic impediments.

\section{Academic Persistence}

In the context of the current study, academic persistence is the participant's ability to persevere academically and strive despite adverse and challenging situations (ButlerBarnes, Chavous, Hurd, \& Farmer, 2013). The participants’ experiences depicted instances in which they demonstrated fortitude and resilience. Moreover, they were able to counteract social and academic barriers and persist academically and cited reasons why they chose to persist. The participants also indicated that their ability to persist academically in the gifted program was not just the result of intrinsic motivation, but was 
due in part to the support of teachers, parents, and friends. As such, the participants frequently used the term 'push' to describe how external motivators influenced their ability to persist.

To take a case in point, Benjamin discussed his approach to persisting academically when he stated, "If there’s ever a point to where it's getting hard for me, I would probably wanna start backing out or something, but I feel like if it gets harder, I need to try to progress and not just stop.” Tony corroborated the statement saying, "If I find something is really difficult, it just makes me try and work harder.” The participant's responses indicated that the more academic challenge they were confronted with, the more compelled they were to achieve. The participants also gave voice to the implications of academic persistence on future pursuits.

For instance, Allen expressed his thoughts about his role as an African American male as it related to academic persistence when he asserted, “To be an African American male, you've gotta do well. It might be challenging down the road, but you gotta get through it. Once you become successful, it'll all pay off.” Allen also considered the role of race as it regarded the effect of academic persistence on future endeavors, when he explained,

It might not be now, but maybe later you'll come across people that'll see you differently because of your skin color, but you can't let what they say determine your future. You've gotta just make it through, and once you become successful, it'll be worth it.

The participants shared similar affirmations as a source of inspiration to negate the hardships experienced in the gifted program. The participants also indicated that they 
were able to persist academically because of the support they received inside and from outside the gifted program.

Throughout the interview process and during focus group discussion, the participants clearly identified multiple instances in which they found teacher and peer relationships to be problematic. In contrast, when identifying support systems within the academic environment, the participants named teachers, friends, and family as those who pushed them to persist academically. As an example, Curtis recalled the prominent role a teacher played in his experience as a gifted student. He said,

Ms. J, she's been one of those people who, you'll be having a bad day, or you'll just be in need of a friend, or support, and she'll just say something to you, and it'll motivate you to do something or brighten your day. She's one of those people who are just a constant support system.

Along the same lines, Felix stated, "my support system in school is my classmates and outside of school is my family." Chris stated, "My whole family pushes me, my mom pushes me a lot too and my dad would be like, you did this, but you can go higher.” Their responses provide strong evidence of supports contributing to the participants' academic persistence.

The participant's academic persistence is evident in their resolve to remain in the gifted program considering the hardships they encountered while participating in the program. In fact, all 10 of the participants indicated they wanted to remain in the gifted program, indicating that the challenges they encountered would not deter them from reaping the benefits associated with participating in a gifted program. As an example, Philip stated, “I wouldn’t want somebody else’s opinion just to push me out, well, 
because I know I’m smart.” Along the same lines, Lee shared, “I don’t feel like anything should cause me to drop out of a program that can help me to succeed later in life.” In another instance, Curtis candidly shared about his resolve to remain in the gifted program when he revealed:

I was really discouraged at the beginning of $7^{\text {th }}$ grade. My GPA dropped. It went from

4.0, to 3.4, to 3.2. It went up after the first few grading periods. I felt like some of the small things, they eventually get to you and it's like, why am I even trying when I feel like I'm not gonna get anywhere? So, then you have to try to overcome it. So, I've never felt, oh, I wanna be exited from this gifted program. The other participants articulated similar experiences that indicate academic persistence as a prominent emergent theme in the experiences of gifted African American males.

The emergent themes in the present study were reflective of the distinctive intricacies inherent to the experiences of gifted African American males. The participant's personal narratives vocalized and interpreted their schooling experiences as adolescents. Their perceptions of acceptance in a gifted program, interpersonal relationships, scholarship and academic resolve, and the role of race in the academic environment bring attention to both the peril and possibility existent in gifted programs for African American males. The following chapter discusses the findings of the study and proposed recommendations for practice and future research toward the betterment of the educational experiences for African American males participating in a gifted program. 


\section{CHAPTER V \\ DISCUSSION AND ANALYSIS OF FINDINGS \\ Introduction}

Much of the scholarly work on African American male achievement belabors the unimpressive narrative of underachievement which contributes to the misconception that the majority of African American males are by in large unsuccessful in school and in achieving future aspirations (McGhee, 2013). Although unsettling, claims regarding African American male underachievement is not without merit. Consider for example evidence that African American males have higher dropout, suspension, and expulsion rates than any subgroup in the United States, fail to reach grade level proficiency in reading, mathematics, history, and science, and are overrepresented in special education programs and low track courses, and are underrepresented in gifted and talented programs (Ford, 2010; NCES, 2009; Skiba, Robert, Michael, Nardo, \& Peterson, 2002). As such, researchers have found that these types of barriers, particularly for African American males in the K-12 educational setting, have potentially harmful implications for negative outcomes well into adulthood, which includes African American males who are gifted (Aud, Fox, \& Kewal-Ramani, 2010; Donner, \& Brown, 2011; Noguera, 2009; Schott Foundation, 2012). 
Schools and educators overlook underserved students such as African American males for participation in gifted programs (Ford, 2013). Although scholars have promoted equitable access for all ethnic students to gifted programs as a means to increase academic engagement and readiness for $\mathrm{K}-12$ instruction and beyond, the absence of ethnic groups in gifted programs, particularly African American males, is cause for concern (Ford \& Moore, 2013; Tomlinson \& Jarvis, 2014). Albeit, special education programs, which includes gifted programming, were designed with the premise of providing equal opportunity and access to students with exceptional needs, gifted African American males continue to be noticeably underrepresented in gifted programs. When they are enrolled in a gifted program, African American males are at greater risk for electing to leave the program (Donovan \& Cross, 2002; Ford, 2012; Ford, Coleman, \& Davis, 2014; McBee, 2006; Olszewski-Kubilius \& Thomson, 2010). Consequently, despite their exceptional intellect and qualifications to participate in a gifted program, gifted African American males are still subject to the same educational obstacles and inequitable practices as African American males in general.

Considering these factors, it may be reasonable to resign African American males to the bleak and oppressive prospects. However, there is unheard and underappreciated counter narrative that provides the basis for reconstructing the dominant misconceptions and social images that have tainted the educational processes and experiences of African American males, specifically those identified as gifted. Based on the findings of my study, the experiences of gifted African American males reveal both victory and victimization. Nevertheless, the resilience and persistence discerned in the voices of gifted African American males offer a promising narrative toward transforming the 
damaging rhetoric and images regarding the social and academic identity of gifted African American males. To expound upon the condition of gifted African American males in the context of the findings, this chapter will (a) present a summary of the study; (b) discuss the findings and literature; (c) provide implications for action and practice; and (d) conclude with recommendations for future research.

\section{Summary of the Study}

U.S. society and educational systems continue to marginalize, stigmatize, and racialize African American males because of entrenched discriminatory practices (Howard, Flennaugh, \& Terry, 2012). As a result, African American males are disproportionately susceptible to the negative influences of race and institutionalized racism entrenched and normalized in contemporary society. Moreover, Howard et al., (2012) asserted that, "Black males often have to actively undo a significant amount of societal and historical stigmas in order to develop a healthy and productive sense of self" (p. 93). Hence, it is not surprising that educational outcomes for African American males reflect disturbing trends in overall academic performance and widening achievement gaps as compared to non-minoritized groups. In fact, Noguera (2008) asserted that the educational system perpetuates race and gender stratification through ability grouping, uneven expectations for learning, and racially distinctive discipline practices, all of which profoundly influences the academic success of African American males. It is also important to note that achievement disparities also exist between high achieving African Americans and White students, specifically African American males who may also qualify for participation in a gifted program (Ford, 2011; Plucker, Burroughs, \& Song, 2010). 
There remains a disproportional representation of African American and Hispanic students in gifted and talented education programs, and African American males are the most underrepresented of all ethnic subgroups (U.S. Department of Education, Office of Civil Rights, 2012). Gifted African American males fall victim to subjective selection practices such as culturally inadequate recruitment and identification criteria, the administration of biased intelligence tests, and flaws in teacher referral processes (Ford \& Whiting, 2010; Matthews \& Kirsch, 2011). Additionally, weak advocacy, lack of knowledge, and the absence of the grit needed to navigate the systems controlling admittance to a gifted program are deterrents for African American males in the application process (Ford, 2011; McBee, 2010). Not only are African American males underrepresented in gifted programs, once enrolled, their socioemotional, psychological, and cultural needs are disregarded and consequently, they are less likely to be retained in the program (Henfield, Washington, \& Owens, 2010; NCES, 2015).

Unlike non-minoritized gifted students, gifted African American adolescent males must develop an academic identity, which takes into consideration how their race, gender, and class will inform and shape their educational experiences (Henfield, Washington, \& Byrd, 2014). Based on the favorable social and academic outcomes of White students, such considerations are not even remotely on the experiential radar of White school- age students, creating a discreet divergence in the way White students and African American students experience a gifted program (Ford, 2014). Specifically, African American males are forced to negotiate feelings associated with "perfectionism, fear of success, underachievement, introversion, non-conformity, heightened selfawareness and feeling different, idealism; justice, concern over world problems, [and] 
empathy” (Scott, 2012, p. 26). While many of these characteristics are common among students identified as gifted regardless of race, they are amplified for gifted African American males. Many of these traits concealed within the psyche of gifted African American males can account for inter and intra personal struggles which requires that their affective needs be addressed (Scott, 2012).

Although the issue of race is an ongoing and prevalent issue as it relates to the schooling experiences of gifted African American males, educators are positioned to initiate policies and practices that have the potential to resonate across all facets of the educational trajectory for this population of students. Primarily, an understanding of the differential needs of gifted African American males may better equip teachers with the culturally informed pedagogical expertise needed to educate African American males. Furthermore, curriculum choices that deviate from traditional mainstream images provide a culturally relevant point of entry into the learning process for gifted African American males to develop a healthy racial and academic identity (Ford, 2011).

Although some researchers suggest that gifted curricula provides an exceptional learning experience for all students regardless of their ethnic background, "very little is known about the extent to which ethnic minority students find academic success in gifted education programs” (Brown, Renzulli, Gubbins, Siegle, Zhang, \& Chen, 2005; Ford, 2013; Henfield, Woo, Bang, 2017, p. 3;VanTassel-Baska \& Brown, 2007). Moreover, the legitimacy of the immense difficulties ethnic minority students experience in predominantly White learning environments is well documented (Covay, 2015; Ford, 2011, 2012, 2013; Webb \& Linn, 2016). Therefore, based on what is known regarding 
the experiences of ethnic minorities in gifted programs, by extension, an examination of the experiences of gifted African American males are needful and worthy of exploration.

Given these factors, the purpose of this study was to explore the lived experiences of gifted African American males and probe how their sense of self-efficacy shaped the manner in which they maneuvered and operationalized the factors influencing their academic persistence. The participants in the study were in grades 6-8, because the voices and experiences of gifted adolescent African American males participating in a gifted program is understudied and underrepresented across the body of literature that has investigated the academic experiences of African American males as a whole (Whiting, 2010). So then, in addition to contributing to and expanding upon the existent knowledge concerning gifted African American males, specifically this study has given attention to the manner in which the intersection of race and gender, coupled with the socioemotional and academic constructs of gifted programs, configures their experiences in a gifted program. Thus, I sought to answer the following research questions:

1. How do gifted African American males understand and experience participation in a gifted program?

2. How do African American males perceive their participation in a gifted program as shaping their sense of self- efficacy and academic persistence?

Based on the aim of the current study, interpretive phenomenology was an appropriate methodology to understand the lived experiences of gifted African American males participating in a gifted program. Phenomenology is "the rigorous and unbiased study of things as they appear in order to arrive at an essential understanding of human consciousness and experience” (Dowling, p. 132, 2005). A core component of 
descriptive phenomenology involves the researcher being able to disregard or bracket prior knowledge and personal bias in relation to the topic of study, whereas interpretive phenomenology expands upon the descriptions of routine life experiences and seeks to derive meaning from them even when it may not be apparent to the subject (Lopez \& Willis, 2004). Also, interpretive phenomenology acknowledges the researcher's presuppositions and the ways in which it informs their interpretation of the subject's narrative (Reiners, 2012). Therefore, interpretive phenomenology was useful in scrutinizing the experiences of gifted African American males and deciphering meaning from various facets of their schooling experiences through the lens of the study's emergent themes.

I triangulated the data for this study using semi-structured interviews and focus groups involving photo elicitation with 10 gifted African American males who participated in a gifted program at the selected study site. In addition to the narrative derived from semi-structured interviews and focus group discussions, photo elicitation prompted participants to deepen their reflections and interpretation of the images in ways that may not immerge using words alone (Harper, 2002). To culminate the data collection process, and provide the participants an opportunity to process and reflect on the focus group discussion, they were given the option of creating an illustration which depicted their experiences as a gifted African American male in a gifted program (Hall, Jones, Hall, Richardson, \& Hodgson, 2007; Harper 2000).

I coded the data using in vivo and focused codes and analyzed utilizing the interpretive phenomenological analysis process from which themes were derived. The major emergent themes were underrepresentation, teacher-student relationships, peer 
relationships, scholar identity, self-efficacy, and academic persistence. The subordinate themes, sense of belonging, unequal treatment, invisibility, and microaggressions and racism, expanded upon and nuanced the cogency of the primary themes. The emergent themes and subordinate themes affirmed, challenged, and at times contradicted previous literature on the experiences of gifted African American males. However, the participant responses provided inarguable evidence of their personal lived experiences participating in a gifted program.

\section{Discussion of the Findings \& Literature}

To appraise the investigation into how gifted African American males, understand and experience participation in gifted program, Critical Race Theory (CRT), utilized as an overarching framework, and Deficit Thinking and Voluntary and Involuntary Groups theories were operationalized as a means of contextualizing their experiences and make meaning of the emergent themes extracted from participant narratives. The theoretical frameworks also served to underpin and explain the phenomenon unique to their experiences as gifted males of color. The participant's narrations melded theoretical assertions with the practical and intimate realities of their daily lives. An interpretation of the findings served to render an accurate representation of the lived experience of gifted African American males through the functionality of the theoretical frameworks as well as to dissect the emergent themes with evidence reported in scholarly literature.

The issue of underrepresentation called attention to the glaring disproportionality of minoritized groups in the gifted program. For example, White students made up $60 \%$ of all students enrolled in a gifted program, compared to 9\% of African American students, of which 4\% are African American males (U.S. Department of Education, Office of Civil 
Rights, Civil Rights Data Collection, 2011-2012). Considering the disparate composition of gifted programs, it makes sense that the participants in the current study were keenly aware that African American males’ participation in the gifted program was a rare occurrence and exclusive opportunity. Underrepresentation transcends the reporting of raw statistical disproportionalities. It is a symptomatic consequence of racially discriminatory practices, which have been normalized in the context of U.S. schooling (Parker \& Lopez, 2003; Lynn \& Parker, 2006).

To further anatomize the issue of underrepresentation, Critical Race Theory asserts that Whiteness, the presuppositions and beliefs that affirm and situate the behaviors of White people as the standard for normalcy in everyday living, gives credence to why minoritized groups are placed at a disadvantage for being identified and enrolled in a gifted program (DeCuir \& Dixson, 2004). For example, Ford, Grantham and Whiting's (2008) examination of teacher referral practices revealed that African American students were consistently under-referred to gifted programs. Along the same lines, African American students were under-referred to gifted programs even when they were equally as qualified as their White counterparts who were consistently over-referred (Grissom \& Redding, 2016). Wright, Ford, and Young (2017) asserted that the over-enrollment of White students in gifted programs created White spaces where Black and Brown students are doubly marginalized based on mainstream associations of race with academic aptitude. Such mindsets perpetuate paradigms of whiteness where differences are perceived as deficits. So then, Harris' (1993) theoretical framework of property, which asserted that whiteness as property legitimizes a perceived position of superiority giving Whites the right to exclude, the right to dispose, the right to elevated status based on 
property acquisition, and the right to take pleasure in all three, drive the momentum for continued underrepresentation in gifted programs. As a result, the historic and seemingly permanent ramification of functionalized whiteness has biased identification practices, effectively excluding culturally different students from gifted programs (Gillborn, 2015).

It is the reality of underrepresentation, Curtis announced, "I looked around and noticed, I'm the only Black person in here.” To expound upon the participant's statement, Anderson (2015) asserted that a white space is a perceived category in which:

Blacks reflexively note the proportion of Whites to Blacks, or may look around for other

Blacks with whom to commune if not bond, and then may adjust their comfort level accordingly; when judging a setting as too white, they can feel uneasy and consider it to be informally off limits. For Whites however, the same settings are generally regarded as unremarkable, or as normal, taken-for-granted reflections of society. (p. 10)

Considering the primacy of white spaces, the participants found it challenging to establish a sense of belonging amidst the cultural homogeneity common in gifted programs. Moreover, the overrepresentation of White students in gifted programs sends an unconscious message about the propensity for students of color to have difficulty surviving in gifted programs as they currently exist. Therefore, to reverse the trend of underrepresentation in gifted programs, gifted identification criteria, teacher referral processes, intelligence tests, retention practices, and deficit orientations would need to deviate from the current social constructs empowered by the interests of White identification norms (Ladson-Billings \& Tate, 1995; Payne, 2011). 
A consequence of underrepresentation is that African American males absorb the socioemotional discomfort of feeling alone and being lonely in the White space of a gifted program. The participants struggled to fit in and establish a sense of belonging. Accordingly, Walton and Cohen (2011) suggested that African American students in general experience greater uncertainty about their sense of school belonging compared to White students. Amari’s comment, “I can’t really fit in anywhere, because I don’t fit in with non-minorities, as far as being gifted”, is a worrisome reminder of the socioemotional trauma associated with belongingness to which African American males are well acquainted in a gifted program.

Sense of belonging is a positive predictor of motivation, engagement, and overall student success (Connell, Halpern-Felsher, Clifford, Crichlow, \& Unsinger, 1995). Also, academic belonging is described as the extent to which one views oneself as fitting in and possessing the qualities to excel academically and be accepted within a given environment (Furrer \& Skinner, 2003; Osterman, 2000). Particularly pertinent to the current study, an ill established sense of belonging within an academic setting becomes hazardous to minoritized groups battling the influences of negative stereotypes (Mendoza-Denton, Downey, Purdie, Davis, \& Pietrzak, 2002; Walton \& Cohen, 2007). Specifically, African American middle school students are more susceptible to risks associated with emotional distress, thoughts of self-harm, violence, substance abuse, and falling grades, in contexts where sense of belonging is not regarded as being essential to their social and academic well-being (Resnick, Bearman, Blum, Bauman, Harris, Jones, \& Udry, 1997; Eccles, Lord, \& Midgley, 1991; Cohen, Garcia, Apfel, \& Master, 2006; Cohen, Garcia, Purdie-Vaughns, Apfel, \& Brzustoski, 2009). By extension, gifted 
African American males in the middle grades invariably contend with social isolation and low social status, that even when initiated by a single instance of exclusion, hampers future attempts to establish acceptance within teacher and peer relationships (Walton \& Cohen, 2011).

The teacher-student relationship plays a crucial role in the education of African American males (Howard, 2008; Milner, 2007). In fact, in schools where teachers do not demonstrate care for racially marginalized students, and are not instructionally competent, they become contributors to existent barriers rather than creating opportunities for social and academic success (Lynn, Bacon, Totten, Bridges, \& Jennings, 2010). Participants in the current study confirmed the importance of care in their experiences in the gifted program and cited comments such as "I feel like teachers care for me and want me to be at the best of my ability", and "my teachers have always pushed me”. Theses utterances provide a glimmer of hope for the long-awaited shift in gifted classroom culture that is fundamental to the success of African American males in a gifted program. However, more pervasive in the current study, were the participant's experiences with and awareness of unequal treatment, which manifested in disparate discipline practices, being talked down to, being perceived as angry, and not being trusted. The participants also experienced feelings of being invisible and made assessments about the role of teacher race in being accepted in the gifted classroom; all of which had implications for the manner in which they developed relationships with their teachers.

Even though many White educators espouse colorblindness as a means of neutralizing their position on race as it relates to their interactions with students of color, 
CRT contends that "in adopting colorblind approaches, White teachers exonerate themselves in the maintenance of racial hegemony, and fail to understand how social and institutional racism pervades the lives of students of color both inside and outside of the classroom” (Allen, 2015, p.72). Also, many White educators adhere to normalized assumptions regarding the social and academic capabilities of African American males and lower their expectations (Klingner, Cramer, and Harry, 2006; Oakes, 2005). In the current study, unequal treatment by the teacher was less evident in relation to the participant's ability to perform academically commensurate with their peers, however the participants expressed considerable differences in the way teachers responded to their behavior compared to the way in which they responded to the same or similar behavior by their White counterparts. As an example, Philip recalled an instance when he said, "You get on the Black kid because he's doing something wrong, but you let the White kids get off, when they're also doing the same thing”. The truism of his comment is affirmed by Irvine’s (1985) seminal study regarding White teacher's handling of Black student behavior. Irvine found that teachers consistently spoke more negatively about Black males than any other group, and teacher's perceptions of their behavior influenced how they evaluated their academic ability. Similarly, when teachers operate through the lens of mainstream assumptions of African American male non-conformity, they often misread their behaviors, thereby facilitating discrepant treatment in discipline practices, as was the case in the current study (Aud, Fox, \& Kewal Ramani, 2010; Gregory, Skiba, \& Noguera, 2010).

Lack of accountability in teacher's executing unequal treatment for African American males, particularly as it relates to prejudicial teacher-student interactions and 
discipline practices, is tantamount to promulgating social and cultural frustration and exclusion. Ogbu and Simmons' (1998) theory of voluntary and involuntary groups provides further explanation. Ogbu and Simmons proposed that African American students exhibit hostility and resentment when they are obligated to conform to conventional ways of behaving in a school setting, whereas non-minoritized gifted students flourish and are content in educational settings normalized by standards of whiteness. Taking into consideration the future implications of unequal treatment, Alexander (2012) suggested that disproportionate race-gendered discipline practices unjustifiably introduce African American males to the school-to-prison pipeline in that the internalization of negatives messages produces reactive and racialized identities (Cokley, McClain, Jones, \& Johnson, 2011).

Historically, teachers have been considered figures of moral authority and as standard bearers of cultural efficacy and societal norms, however, student's perceptions of teachers as symbols of integrity in the classroom setting have been challenged (Allen, 2015; Pace \& Hemmings, 2007). In light of the perceived negative behaviors of African American males, it makes sense that perceptions of gifted African American males in the present study include a source of acrimony and mistrust. As a result, they dealt with making sense of irreconcilable claims regarding their teachers such as, "I feel like teachers care for me”, versus “They don’t trust us or want to be by us” validating the assertions of Pace and Hemmings (2007) and Allen (2015). According to Gregory and Ripski (2008), while the lack of trust teachers demonstrated for African American males hindered the development of the teacher-student relationship, their trust in teachers to advocate on their behalf was equally as consequential, yet has received little attention. 
The findings also indicated that gifted African American males’ perceptions of unequal treatment exacerbate feelings of invisibility. Invisibility in the context of the present study was considered the inner conflict emanating from the feeling that one's gifts, capacities, and individuality, are not valued or recognized because of the intrusion of racism and prejudice. Experiencing invisibility also drives African Americans to makes themselves visible in order to achieve acceptance (Franklin \& Boyd-Franklin, 2000), much like the study's participant Benjamin did when he inserted himself into peer groups without invitation to find acceptance among his gifted classmates. The concept of invisibility confirmed the findings as reported by the participants. They made concrete associations with feelings of invisibility. For example, they literally felt overlooked based on their physical placement in the classroom as evidenced by their verbal and pictorial representations. Additionally, they believed invisibility also encompassed instances of being ignored when they attempted to participate in class activities and when they experienced the void of culturally relevant images, text, and curriculum in the gifted classroom. For instance, they struggled to recall occasions when they felt their ethnicity was represented or valued in their gifted classroom besides annual references to Black History month. They translated their feelings of invisibility to mean, if you do not see me, you do not accept me. In sum, competent teachers of African American students provide them with what they need as individuals, respect, and integrate the value of the student's culture, and see themselves as an essential contributor in their holistic development (Bacon, Totten, Bridges, \& Jennings, 2010).

Drawing from their personal experiences regarding teacher-student relationships, in the present study, gifted African American males believed that having a teacher of the 
same race would ameliorate the issues of unequal treatment and invisibility enacted by White teachers. Even when gifted African American males reported never having an African American teacher, they considered the presence of an African American teacher in a gifted class or any class in the school, a symbol of hope for African American students in general, as a means to improve schooling experiences for students of color in the classroom. They explained their reasoning by stating, "they would understand me more”, and "I could probably be more open to them.” They also recognized them as an example for students of color to affirm the plausibility of successfully achieving future aspirations. Participants taught by an African American teacher recalled specific feelings about their experiences under the instruction of an African American teacher. Proclamations such as, "I was comfortable around her", "She made me feel included", "I wasn't overlooked", and "It was my best year in school”, give insight into the stark differences in the perceptions and experiences of African American males compared to non-minoritized students in a gifted classroom as moderated by teacher-student race congruence.

In alignment with the findings, bureaucratic representation theory suggests that teachers of color are more apt to advocate and exercise discretion with students of their same ethnic background, and these preferences are the same for White teachers with White students (Grissom \& Redding, 2016). Along the same lines, teachers may embrace and diagnose giftedness differently in students from other races based on prejudicial judgments of student expectations (Gershenson, Holt, \& Papageorge, 2015; Grissom, Nicholson-Crotty, \& Nicholson-Crotty, 2009). This is not to suggest that White teachers cannot engage gifted African American males in meaningful educational experiences. 
However, changing the dominant socioemotional conditions in gifted programs would require first that "teachers subvert hegemonic school systems in more subtle ways through advocacy and activism for marginalized students, or acting as cultural brokers by helping students navigate the institutional norms” (Grissom \& Redding, 2016, p. 73). A shift in redefining institutionalized norms in the gifted classroom setting would not only reconstruct the context of teacher-student relationships, but would also reconfigure the structure of peer relationships for gifted African American males in the gifted program.

Scott (2012) purported that gifted students wrestle with their giftedness because they have a need to fit in and feel connected to others in their peer group. African American students in particular require affiliation and would elect for acceptance rather than be shunned or isolated by their peers (Scott, 2012). Acceptance among peers is especially stressful for African American students because many African American students are communal in the ways they orient themselves in a school setting (Ford, 2011). Accordingly, the participants in my study placed emphasis on their feelings of acceptance in the gifted program by their gifted peers. While the participants indicated they were able to navigate the gradations of their peer relationships with non-minoritized students, they also reported that it was common to be excluded from a group in which their giftedness was on display. They were cognizant of the ongoing negotiation between acceptance and racism and microaggressions as the primary impediments to developing a legitimate relationship. The participants offered specific examples of the pervasiveness of the existence of microaggressions vocalized by their peers. Based on race, ethnicity, gender, or a combination of all three, microaggressions are verbal, actionable, and attitudinal acts of disrespect, subtly or overtly executed, that often go undetected or 
unaddressed (Sue, 2010, Valencia, 2010). The microaggressions participants encountered were reflected in queries such as "Why are you in this GT class?" and "I thought you had to pass the GT test to get into this class, or did someone take it for your?” which give validity to the latent assumptions non-minoritized students possess regarding the abilities of gifted students of color (Stambaugh \& Ford, 2015).

In the same vein, blatant racist comments, although just as harmful as microaggressions, were often dismissed, understated, or ignored when perpetrated by same-age peers (Stambaugh \& Ford, 2015). Participants in the current study experienced direct and inferred racism from their peers. As reported by the participants, comments about their physical features and stereotypical references associated with race, were among the reasons they felt unaccepted and confessed, "It just made me really sad"; "I just didn’t feel accepted”. In Galbraith’s (1985) prominent study of gifted children’s most common complaints, the researcher found one of them to be the gifted child's inescapable awareness of being different and being misunderstood. Galbraith’s conclusions did not consider the strife experienced by students of color dealing with racism in a gifted program. Using Galbraith's findings as a premise, it is expected that African American males experience compounded challenges in being respected, accepted, and understood in a gifted program.

Exploring how gifted African American males understand and perceive their participation in a gifted program exposed the obstacles that they must overcome to capitalize on the benefits of being in a gifted program. However, inflexible schooling constructs, informed by racialized norms, have created an educational labyrinth from which gifted African American males have struggled to emerge. Based on the claims of 
the participants in the current study, although severely underrepresented, African American males who are identified and participate in a gifted program, unknowingly enroll in potentially one of the most daring undertakings of their educational career.

In the current study, participant responses shed light on the academic behaviors of gifted African American males in shaping their sense of self-efficacy and academic persistence. African American males in general have overwhelmingly experienced negative outcomes in school as compared to their White counterparts (Moore \& Owens, 2008; Ford, 2010). In the case of gifted African American males, underachievement in school often replicates the frequently reported underachievement of non-gifted African American males (Ford \& Moore, 2013). Ford, Grantham and Whiting (2008) suggested that the pressures of acting White (e.g., studying, getting good grades, certain style of dress) or acting Black (e.g., verbosity, poor academic performance, being rebellious), contributed to underachievement. Scholars have also attributed the underachievement of African American males in gifted programs to social, cultural, family, school, and personal factors (Ford, 2010, 2013; Hébert \& Schreiber, 2010; Henfield, Moore, \& Wood, 2008; Moore \& Owens, 2008). The same scholars contended that underachievement is also a function of educator dispositions, attitudes, and culturally deficient policies, and practices that perpetuate poor performance for students of color. This includes deficit ideologies that frame the way in which educators view African American males. Meaning, educators who conform to deficit ideologies place blame on African Americans for their social and academic plight, embrace stereotypical views about students from diverse backgrounds, and lower their expectations based on race, 
thereby contributing to the conditions that promote underachievement (Ford \& Grantham, 2003; Olivos, 2006).

From a different perspective, deconstructing deficit thinking has the potential to reverse the academic outcomes for African American males, especially those who are gifted, both identified and unidentified. Drawing upon Mickelson’s (1990) seminal study on the academic achievement gap between White and African American students, African American students have been poised for an academic turnaround, but have been awaiting culturally apprised educators and the supportive social and academic conditions necessary to facilitate a new direction in their academic trajectory. Mickelson found that despite attitude-achievement paradox, the discrepancy between student beliefs and corresponding behavior, Black students expressed a high regard for the value of education even when their academic performance did not reflect their expressed belief (Ford, Grantham \& Whiting, 2008). Counter narratives to the underachievement of African American males however, served as a welcomed contradiction in the narratives provided by the participants in the current study as they recounted high levels of academic performance in the gifted program.

Gifted African American males who demonstrate self-efficacious behaviors that lead to high levels of academic performance, and persist beyond adversities that otherwise would lead to their academic demise, possess scholar identity (Whiting, 2006). The participants in the present study characterized themselves as confident and intelligent. They believed their academic abilities were equivalent to that of their White counterparts. The participants were conscientious about earning good grades and made the appropriate adjustments if they underperformed, particularly if a lower grade was the 
result of their own negligence. They regarded all academic achievements as a significant accomplishment. Their self-perceptions as it related to performance in the gifted classroom epitomized characteristics consistent with scholar identity. To expand upon the development of scholar identity, Whiting (2009b) noted that pride, self-esteem, and self-efficacy for African American males is most often associated with their exceptional acumen in athletics and the arts, however shifting the focus to the academic setting has enduring positive implications for gifted African American males. In fact, Whiting (2009b) stated that, "self-efficacy lays the cornerstone of a scholar identity", which further solidified the salience of recognizing the participant's scholar identify in shaping their academic persistence (p. 228).

The findings in the current study mirrored the assertions of Bandura’s (1977) and ascribed high priority to the role of self-efficacy in educational outcomes based on one's belief that they are competent and capable. Benjamin's declaration, “I worked hard...so academically, I feel confident”, aligned with Whiting's (2006) conceptual model, which included academic self-confidence as a characteristic of scholar identity. Whiting found that high self-efficacy aided African American males in being resilient and persisting in the face of adversity. Bearing out Whiting's assertions, in the current study, the participant's resolve to persist academically demonstrated their will to reject racial stereotypes, overcome setbacks, and build upon the efficacious behaviors and habits that facilitated their current academic successes. So too, the participant's high level of selfefficacy substantiated the findings of Uwah, McMahon, and Furlow's (2008) study in which they determined that self-efficacy was more significant to academic achievement than self-concept and self-esteem. In fact, Jonson-Reid, Davis, Saunders, Williams, and 
Williams (2003), suggested that beliefs about self-efficacy were a stronger predictor of academic achievement for African American adolescents than self-esteem.

The participants' desire to persist academically was subject to their need to achieve and persevere in the gifted setting. McClelland's (1961) need to achieve theory confirmed the motivation and drive of the participants as another indicator of their scholar identity. Similarly, their academic persistence was a motivational construct associated with their academic performance (Butler-Barnes, et al., 2013). Earlier studies on academic persistence also associated academic persistence with academic outcomes related to scholastic goal-setting and high school retention rates (Hardre \& Reeve, 2003, Neblett, Philip, Cogburn, \& Sellers, 2006). The basis of the earlier studies on academic persistence coincided with the participant's understanding that their academic persistence had directs implications for sustaining current academic performance, but also for achieving future aspirations beyond the gifted program as evidenced by Allen when he stated "Once you become successful, it’ll all pay off."

Participants in the current study indicated that they were able to persist academically because they were pushed. They used the word push to describe external support and reinforcement, which enabled them to persist academically despite the social and emotional vicissitudes experienced in the gifted program. The participants acknowledged that there were teachers, friends, and especially family members who held them accountable, reinforced the ability to achieve, and consistently held high expectations of them. They provided examples of how certain teachers and peers affirmed them as individuals and served as a sounding board when they were confronted with difficult situations. Although peers are influential during adolescence, familial 
support of academic achievement among African Americans plays an important role in encouraging them to do well in school (Kerpelman, Eryigit, \& Stephens, 2008). Indeed, Chris' comment “my whole family pushes me... my dad would be like you did this, but you can go higher” confirms the significance of parent-adolescent relationships as important to academic persistence.

The participant's exhibited an extraordinary ability to traverse social and emotional obstacles exclusive to gifted African American males. Considering their unparalleled and provocative educational experiences within the gifted African American males in the present study were able to denounce the oppressive dominant narratives often associated with African American males and reformulated an intellectualized profile consistent with their scholastic strengths. Meaning, the participants were able to persist academically because of the way they perceived themselves as possessing characteristics of scholar identity necessary for academic success. They were able to remain in the gifted program and defy social and racial indignation because their higher levels of self-efficacy dictated the need to be successful and capitalize on their exceptional academic abilities. The participants' belief that they were equally as capable as their non-minoritized peers, helped them to assert themselves academically and disempower the impact of microaggressions aimed at invalidating their intelligence. In so doing, the participants redefined the social and academic boundaries imposed from the racialized and inequitable practices pervasive in gifted programs.

So then, gifted African American males are able to persist despite the inescapable racial, social, and emotional hardship they encounter participating in a gifted program. Arguably the idea of academic persistence in this study is an indicator of resolve distinct 
to gifted African American males to resist contributing to and conforming with defaming narratives yoked to them and African American males in general. To further explore the notion of persistence as a means of racial and social resistance, Butler-Barnes, Chavous, Hurd, and Varner (2013) used the Integrative Model for the Developmental Competencies of Minority Children to examine how personal and cultural assets buffered and moderated the negative impact of discrimination and developmental competencies on academic persistence (García-Coll, Lamberty, Jenkins, McAdoo, Crnic, Wasik, \& García, 1996). Butler-Barnes, Chavous, Hurd, and Varner (2013) found that the presence and influence of racial discrimination in the academic environment significantly reduced academic persistence. In an earlier study, Sellers, Copeland-Linder, Martin, and Lewis (2006) concluded that there was a significant correlation between racial discrimination and lower psychosocial welfare. Considering these findings, the prospect for gifted African American males to succeed is glim at best. However, the gifted African American males in this study resisted succumbing to the deep-rooted bias and discriminatory practices ingrained in schooling social structures. More succinctly stated, gifted African American males' academic persistence served as a proclamation in resisting the deficit-based academic and social expectations imposed upon them by society thereby rewriting the deleterious and dominant narratives to more accurately reflect their strengths and contributions.

In summary, the purpose of my study was to explore the phenomenology of the lived experiences of gifted African American males in grades 6-8 participating in a gifted program. Using the interpretive phenomenological approach, the study attempted to unearth the lived experiences of gifted adolescent African America males to understand 
how they experience their participation in a gifted program and perceive their sense of self-efficacy as shaping their academic persistence. Since, interpretive phenomenology seeks to delve into the intricacies of subjects of a study as individuals, this study sought to make meaning of the participant's experiences as individuals, but also to identify commonalities in their experiences as reflected in the literature.

The six major themes emerged from the data including underrepresentation, teacher-student relationships, peer relationships, scholar identity, self-efficacy driven by the need to succeed and academic persistence. Subordinate themes, sense of belonging, invisibility, unequal treatment, and microaggressions and racism further refined the major themes. The findings suggested that placed in a position of social, emotional, and academic vulnerability, the gifted African American males in the current study defied the dominant narratives commonly associated with their disengagement and persevered through the socioemotional landmines of unacceptance, invisibility, and racism. They were able to disavow the rampant negative images and lowered expectations charged to them as students of color in a gifted program, and persist academically. They demonstrated that internal mechanisms (e.g., self-efficacy, motivation, persistence) have the power to mitigate practices within gifted programs that are discriminatory and injurious to their academic and social development. Moreover, the participants in my study constructed a blueprint from which educators and policymakers can reevaluate the systems that dictate what it means to African American and male in a gifted program.

\section{Implications for Action and Practice}

As the changing cultural landscape of our nation becomes more diverse, it is incumbent upon the educational system to develop quality programing for gifted students 
from all ethnic backgrounds (Olszewski-Kubillius \& Clarenbach, 2014). However, a major fault with gifted education is the underrepresentation of culturally diverse students, particularly African American (Henfield, Washington, \& Byrd, 2014). The Office of Civil Rights (2012) reported that in the United States, African Americans made up 19\% of the total school population, but represented $10 \%$ of students in gifted education. As a result of the ethnic imbalance for both student and other school official role groups, minoritized students have reported having negative experiences (Allen, 2015). Issues with identification and recruitment practices are responsible for contributing to the underrepresentation of low-income and minoritized students to gifted programs, which often includes African American males (Olszewski-Kubillius \& Thomson, 2010). The overreliance on culturally biased intelligence tests and flawed teacher nomination and referral practices contribute to the underrepresentation of African American males in gifted programs. In fact, research reveals significant differences in teacher referral rates based on student race and background (McBee, 2006). As a result, African American males, even when they are qualified, have less of an opportunity to partake in the enhanced benefits that come from participating in a gifted program (Henfield et al., 2014).

Exacerbating the disproportionality in the enrollment and retention of African American males in gifted programs are social and psychosocial factors that contribute to and perpetuate an emotionally and socially substandard and deleterious learning environment for African American males in gifted programs (Ford et al., 2008). For example, African American males may be unaccepted, ostracized, or accused of acting White because racialized norms have infiltrated the learning environment in gifted 
programs (Grantham, 2004). While denying African American males the opportunity to participate in a gifted program based on systemic flaws within the educational system governed by race, Henfield, Woo, and Bang (2017) asserted that "it may not necessarily be a given that learning in gifted education programs, an example of majority-White learning context, is academically beneficial for ethnic minority students” (p. 61). In order to revolutionize the current circumstances of African Americans males in gifted programs requires restructuring identification practices, evaluating socioemotional factors, and engaging culturally relevant policies and procedures in academe.

This study advocates a cultural and strengths-based approach to addressing the needs of gifted African American males as participants in a gifted program. However, to effectively identify and retain African American males to gifted programs, a divorce from the limitations of deficit thinking is required of educators (Howard, 2013). Functioning under deficit ideologies acts as the medium by which racially prejudiced beliefs about students of color and self-fulfilling behaviors persist (Olivos, 2006). It is important for educators to understand how deficit thinking surfaces in school settings. Educators must also be equipped to eradicate deficit thinking and hold other stakeholders in the school setting accountable for repudiating deficit orientations.

Since culture matters in educating African American males, it is vital that educators obtain cultural knowledge about African American males and adjust pedagogy accordingly (Milner, 2010). The experiences of the participants illuminate the imperative for teachers of gifted students to examine and establish a culturally dynamic classroom culture and make a concerted effort to ensure gifted African American males are socially, emotionally, and academically acclimated in a gifted program. 
In order to ensure that gifted African American males have access to a culturally rich cadre of educators who are prepared to address the cultural nuances and sensitivities of this distinct student population, teacher preparation programs will need to undergo an andragogical shift to produce teachers who are well-equipped to effectively operate within the context of increasingly diverse student populations. By 2050 nearly two-thirds of students in American schools will be students of color, yet the majority of teachers are White (Goldenberg, 2013). The dominance of cultural diversity in schools warrants the redesign of teacher preparation programs to be consistent with the recommendations of scholarly literature that provides a culturally sound pedagogical roadmap divergent from tradition programs which have proven to be ineffective in preparing teachers. Additionally, teacher preparation programs are needed to broaden the cultural lens through which White teachers view students of color. For instance, White teachers are minimally aware of the presence and influence of racism and discrimination, they bring little understanding of overt inequalities other than the obvious behaviors associated with deficit ideologies, they have limited knowledge of communities of color and in some instances fear them, and often lack awareness of themselves as possessing cultural capital that may work against students of color (Sleeter, 2008). These reasons alone, give urgency to the imperative for quality teacher preparation programs.

Once schools realize an abatement of deficit ideologies, educators and policymakers can reasonably give attention to assessing the issues of cultural equity associated with identification practices. Proper identification of gifted African American males necessitates that lawmakers arrive at expanded and culturally informed definitions of giftedness, taking into consideration the atypical characteristics demonstrated by 
students of color who are gifted (McClain \& Pfeiffer, 2012). Accordingly, findings from my study regarding the underrepresentation of African American males in gifted programs adduces that based on low enrollment in gifted programs, access continues to be problematic. Theoretically, gifted programs serve high-achieving students across all ethnic backgrounds. However, "variance patterns in the establishment and funding of gifted education may suggest that not all students have access to gifted education” (Kettler, Russell, \& Puryear, 2015, p. 100). With sufficient funding educators can focus their efforts on developing inclusive identification criteria and accessing assessment tools void of cultural biases.

Gifted African American males identified for and participating in a gifted program experience undue stress from the socioemotional strain of being African American, male, and gifted within the context of a predominantly White learning environment in that the socioemotional well-being of gifted African Americans plays a central role in their academic success (Winsler, Karkhanis, Kim, \& Levitt, 2013). Specifically, discrimination within school, differential treatment, low expectations, sense of belonging, racial identity, self-concept, and sense of self-efficacy contribute to the how African American males experience and exist in a gifted program (Ford, 2008, 2011, 2013; Ford \& Whiting, 2010; Matthews \& Kirsch, 2011). Therefore, educators bear the burden of creating a specialized and nurturing environment in which gifted African American males can thrive while mitigating sources of socioemotional trauma. Accordingly, Henfield, Washington and Byrd (2014) suggested that school counselors in particular engage in ongoing professional development relevant to the experiences of 
gifted African American males to equip themselves with the cultural competencies vital to their social and academic progress.

Best practices in educating gifted African American males hinges on the readiness of policymakers and school officials to recast a vision for how to transform existing policies and procedures dramatically that govern the ethical and practical framework of gifted programs. Current legislation and school policies have failed to address the lingering inequities that exist in gifted programs, except to provide limited funding (e.g., Javits Act) with minimal oversight on its impact for students of color (Swanson, 2007). For example, schools need policies and practices that support the integration of culturally relevant curriculum, to recruit more teachers of color, to evaluate teacher competency in schools with higher minority populations, and provide them with rich professional development experiences designed to meet the needs of gifted African American males (Wright, Ford, \& Young, 2017).

\section{Recommendations for Future Research}

The current study focused on gifted African American males in grades 6-8 to understand how they experienced gifted program and how they perceived their sense of self-efficacy as shaping academic persistence. Previous research on this topic has focused primarily on high school and college aged students (Ford, Moore, Milner, 2005). Nevertheless, the findings of my study of adolescent African American males related to Noble’s (2011) study on African American males’ self-efficacy affecting task choice and the motivation expended to persist in a collegiate mathematics class. Noble found that self-efficacy had substantial influence on academic outcomes. Noble claimed that based

on the profound roles of sense of self-efficacy on positive academic outcomes, school and 
district leaders should give greater attention to building personal assets and providing models of success to support an increased sense of self-efficacy. The finding of the current study suggests that although gifted African American males endure a myriad of challenges in a gifted program, maintaining scholar identity is influential in shaping selfefficacy, which led the participants in the present study to persist and achieve academic success similar to the participants in Noble's (2011) study.

Scholars and practitioners must acknowledge that gifted African American males are in an ongoing battle to receive an education comparable to their non-minoritized peers in a gifted program, and recognize that doing so requires immediate action if we are to interrupt and reverse the academic trajectory of gifted African American males. Future research should address how to reimagine ways of defining and conceptualizing African American males in general. With regard to identification practices, research suggests a need to know more about teacher self-efficacy and the extent to which teacher efficacy influences the nomination and referral process. Furthermore, considering the findings of the current study, continued studies of socioemotional factors, particularly sense of belonging, moderating microaggressions, and self-efficacy as it relates to the relationships with school officials such as counselors and administrators and how those relationships contribute to academic success are needed.

\section{Concluding Remarks}

I sought to examine the lived experiences of gifted African American males participating in a gifted program and to understand how they perceived their sense of self-efficacy as shaping their academic persistence. Although scholarly literature has documented the condition of gifted African American males in gifted programs, the 
research conducted on gifted African American males, while growing is still limited. Gifted African American males and the circumstances surrounding them represent the ever-present reality of the constructs of race in society under which they form their perceptions of self and life as a gifted middle school student. Considering the gravity of the daily challenges faced by gifted African American males, this study probing the social and academic mechanisms influencing their success was appropriate and vital.

This phenomenological study on the lived experiences of gifted African American males amplified their hushed voices and exploited the failure of academic institutions to advance beyond the antiquated, discriminatory, and racialized educational infrastructure and systems that have and continue to marginalize them. Gifted African American males represent an untapped sector of society in which giftedness may look and sound culturally different from the descriptors of giftedness that have long excluded them from gifted programs. It is my hope that the findings of this study will reveal implications of the values, beliefs, and actions of educators and inspire a self-assessment of their roles in the achievement of gifted African American males and become agents of change to ensure future gifted African American males can access and enjoy the benefits of participating in a gifted program. 


\section{REFERENCES}

Abiola T., \& Udofia O. (2011). Psychometric assessment of the Wagnild and Young's resilience scale in Kano, Nigeria. BMC Res. Retrieved from

http://www.ncbi.nml.nih.gov/pmc/articles/PMC466122

Allen, Q. 2015. Race, culture and agency: Examining the ideologies and practices of U.S. teachers of Black male students. Teaching and Teacher Education 47(1), 71-81.

Alexander, M. (2012). The new Jim Crow: Mass incarceration in the age of Colorblindness. New York, NY: New Press [Jackson, TN]: Distributed by Perseus Distribution, c2012. Rev. ed.

Anderson, E. (2015). The white space. Sociology of Race and Ethnicity, 1(1), 10-21.

Anquiano, L. T. (2003). Under representation of minority students in gifted and talented education. Multicultural Education, 11(1), 32-34.

Archambault, F.X., Westberg, K.L., Brown, S., Hallmark, B.W., Emmons, C., \& Zhang, W. (1993). Regular classroom practices with gifted students: Results of a national survey of classroom teachers (RM93102). Storrs, CT: The National Research Center on the Gifted and Talented, University of Connecticut.

Aud, S., Fox, M., \& Kewal-Ramani, A. (2010). Status and trends in the education of 
racial and ethnic groups (NCES 2010-015). U.S. Department of Education, National Center for Education Statistics. Retrieved June 21, 2013, from http://nces.ed.gov/pubs2010/2010015.pdf

Bacon, J.N., Totten, T.L., Bridges, T.L., \& Jennings, M. (2010). Examining teachers' beliefs about African American males in a low-performing high school in an African American school district. Teachers College Record, 112(1), 289-330.

Baker, A. (2013). Gifted, talented and separated: In one school, students are divided by the gifted label-and race. The New York Times. Retrieved from https//www.nytimes.com

Baldwin, A.Y. (2004). Culturally diverse and underrepresented populations of gifted students: Essential readings in gifted education: Volume 6. Thousand Oaks, CA: Corwin Press.

Bandura, A. (1977). Self-efficacy: Toward a unifying theory of behavioral change. Psychological Review, 84, 191-215.

Bandura, A. (1986). Social foundations of thought and action: A social cognitive theory. Englewood Cliffs, NJ: Prentice Hall.

Bandura, A. (1993). Perceived self-efficacy in cognitive development and functioning. Educational Psychologist, (28)2, 117-148.

Bandura, A., Barbarnelli, C., Caprara, G.V., \& Pastorelli, C. (2001). Self-efficacy beliefs as shapers of children's aspirations and career trajectories. Child Development, (72)1, 187-206.

Banik, B.J. (1993). Applying triangulation in nursing research. Applied Nursing Research, 6(1), 47-52. 
Barbour, R. S. (2001). Checklists for improving rigor in qualitative research: A case of the tail wagging the dog. British Medical Journal, 322(1), 1115-1117.

Barlow, K., \& Dunbar, E. (2010). Race, class, and whiteness in gifted and talented identification: A case study. Berkley Review of Education, 1(1), 63-85.

Barnes, R. (1990). Race consciousness: The thematic content of racial distinctiveness in critical race scholarship. Harvard Law Review, 103(1), 1864-1871.

Barton, P.E., \& Coley, R.J. (2009). Parsing the achievement gap II: Policy information report. Education Testing Service, 1(1), 1-38.

Bartunek, J.M., \& Seo, M.G. (2002). Qualitative research can add new meanings to quantitative research. Journal of Organizational Behavior, 23(2), 237-242.

Bednall, J. (2006). Epoche and bracketing within the phenomenological paradigm. Issues in Educational Research, 16(1), 123-138.

Bernard, H.R. (1995). Research methods in anthropology: Qualitative and quantitative Approaches ( $2^{\text {nd }}$ ed.). Walnut Creek, CA: Altamira.

Bernard, H.R. (2011). Research methods in anthropology: Qualitative and quantitative approaches (5 ${ }^{\text {th }}$ ed.). Walnut Creek, CA: AltaMira Press.

Bhatt, R. (2011). A review of gifted and talented education in the United States. Education Finance and Policy, 6(4), 557-582.

Boeije, H.R. (2010). Analysis in Qualitative Research. Sage Publications, London.

Bonner, F.A. (2000). African American giftedness: Our nation’s deferred dream. Journal Of Black Studies, 30(5), 643-663.

Bonner, F.A. (2003). To be young, gifted, African American, and male. Gifted Child Today, 26(2), 26-39. 
Borland, J.H., Schur, R., \& Wright, L. (2000). Economically disadvantaged students in a school for the academically gifted: A positivist inquiry into individual and family adjustment. Gifted Child Quarterly, 44(1), 13-32.

Borland, J.H. (2003). Gifted education without gifted children: The case for no conception of giftedness. In R.J. Sternberg \& J.E. Davidson (Eds.), Conceptions of Giftedness (pp. 1-19). New York: Cambridge University Press.

Brooms, D. R. (2015). We didn’t let the neighborhood win: Black male students’ experiences in negotiating and navigating an urban neighborhood. The Journal of Negro Education, 84(3), 269-281.

Brown, S.W., Renzulli, J.S., Gubbins, E.J., Siegle, D., Zang, W., \& Chen, C.H. (2005). Assumptions underlying the identification of gifted and talented students. Gifted Child Quarterly, 49(1), 68-79.

Brown, J.S., Collins, A., \& Duguid, P. (1989). Situated cognition and the culture of learning. Educational Researcher, 18(1), 32-41.

Brulles, D., Saunders, R., \& Cohen, S.J. (2010). Improving performance for gifted students in a cluster grouping model. Journal for the Education of the Gifted, 34(2), 327-350.

Brüill, M. (2009). Academic self-concept and change of reference group: Studying contrast and assimilation effects and factors that influence self-concept changes after changing the reference group. Unpublished dissertation. University of Trier, Trier.

Burrows D., Kendall, S. (1997). Focus groups: What are they and how can they be used in nursing and health care research?. Social Sciences in Health 3(1), 244-253. 
Butler-Barnes, S.T., Chavous, T.M., Hurd, N., \& Varner, F. (2013). African American adolescents' academic persistence: A strength-based approach. Journal of Youth and Adolescence, 42(9), 1443-1458.

Byrne, M. M. (2001). Evaluating findings of qualitative research. AORN Journal, 73(1), 703-704.

Caelli, K. (2000). The changing face of phenomenological research: Traditional and American phenomenology in nursing. Qualitative Health Research, 10(1), 366377.

Callahan, C. M. (2005). Identifying gifted students from underrepresented populations. Theory into Practice, 44(2), 98-104.

Card, D., \& Guiliano, L. (2014). Does gifted education work? For which students? (Working Paper No. 20453). Cambridge, MA: National Bureau of Economic Research. Retrieved from http://www.nber.org/papers/w20453

Carpenter, D. M., \& Ramirez, A. (2007). More than one gap: Dropout rate gaps between and among black, Hispanic, and white students. Journal of Advanced Academics, 19(1), 32-64.

Charmaz, K. (2014). Constructing grounded theory ( $2^{\text {nd }}$ ed.). Thousand Oaks, CA: Sage. Choi, N. (2005). Self-efficacy and self-concept as predictors of college students' academic performance. Psychology in the Schools, (42)2, 197-205.

Coffey, A., \& Atkinson, P. (1996). Making sense of qualitative data: Complementary research strategies. Thousand Oaks, CA: Sage.

Cohen, G. L., Garcia, J., Apfel, N., \& Master, A. (2006). Reducing the racial 
achievement gap: A social-psychological intervention. Science, 313(1), 13071310.

Cohen, G. L., Garcia, J., Purdie-Vaugns, V., Apfel, N., \& Brzustoski, P. (2009). Recursive processes in self-affirmation: Intervening to close the minority achievement gap. Science, 324(1), 400-403.

Cokley, K., McClain, S., Jones, M., \& Johnson, S. (2011). A preliminary investigation of academic disidentification, racial identity, and academic achievement among African American adolescents. High School Journal, 95(2), 54-68.

Colangelo, N., Assouline, S., \& Gross, M. (Eds.). (2004). A nation deceived: How schools hold back America's brightest students. Iowa City, IA: The Belin Blank Center for Gifted Education and Talent Development.

Coleman, M.R. (2003). The identification of children who are gifted. Arlington, VA: ERIC Clearinghouse of Disabilities and Gifted Education. (ERIC Digest \#644).

Collier, J. (1957). Photography in anthropology: A report on two experiments. American Anthropologist, 59(1), 843-859.

Colucci, E. (2007). Focus groups can be fun: The use of activity oriented questions in focus group discussions. Qualitative Health Research, 17(10), 1422-1433.

Connell, J. P., Halpern-Felsher, B. L., Clifford, E., Crichlow, W., \& Usinger, P. (1995). Hanging in there: Behavioral, psychological, and contextual factors affecting whether African American adolescents stay in high school. Journal of Adolescent Research, 10(1), 41-63.

Covay, E. (2015). Racial composition and racial differences in opportunities to learn. Journal of Education for Students Placed at Risk, 20(3), 238-262. 
Craig, R., Kretsedemas, P., \& Gryniewski, B. (1997). Picturing African-Americans: Readers reading magazine advertisements. Visual Sociology, 12(1), 28-58.

Creswell, J. W. (1998). Qualitative inquiry and research design: Choosing among five traditions. Thousand Oaks, CA: Sage Publications.

Creswell, J. W. (2007). Qualitative inquiry and research design: Choosing among five traditions ( $2^{\text {nd }}$ ed.). Thousand Oaks, CA: Sage.

Creswell, J.W. (2009). The selection of a research design. In Research design: Qualitative, Quantitative, and Mixed Methods Approaches ( ${ }^{\text {rd }}$ ed.). Thousand Oaks, CA: Sage Publications.

Creswell, J.W. (2014). Research design: Qualitative, quantitative, and mixed methods approaches $\left(4^{\text {th }}\right.$ ed $)$. Thousand Oaks, CA: Sage Publications.

Creswell, J.W., \& Miller, D.L. (2000). Determining validity in qualitative research. Theory into Practice, 39(3), 124-130.

Cross, W. E., Jr., \& Vandiver, B. J. (2001). Nigrescence theory and measurement: Introducing the Cross Racial Identity Scale (CRIS). In J. G. Ponterotto, J. M.

Casas, L. A. Suzuki, \&

C. M. Alexander (Eds.), Handbook of multicultural counseling ( $2^{\text {nd }}$ ed., pp. 371393). Thousand Oaks, CA: Sage.

Curry, T., Strauss, J., \& Strauss, R. (1986). A little pain never hurt anybody: a photoessay on the normalization of sports injuries. Sociology of Sport Journal, 11(2), $192-208$

Dahlberg, K., Drew, N., \& Nystrom, M. (2008). Reflective lifeworld research ( $2^{\text {nd }}$ ed.). Studentlitterayur: Sweden.

Darder, A. (2011). A Dissident Voice: Essays on Culture, Pedagogy, and Power. New 
York: Peter Lang.

Darity, W.A., Jr. (2009). Desegregated schools with segregation education. In C. Hartman \& G.D Squires (Eds.), The integration debate: Futures for American cities (pp. 99-117). New York, NY: Routledge.

Data Books (2015). Jefferson County Public Schools Data Management Planning and Program Evaluation Division. Retrieved from https://www.jefferson.kyschools.us/department/data-management-planning-andprogram-evaluation-division.

Davis, G., \& Rimm, S. (2004). Education of the gifted and talented (5th ed.). Needham Heights, MA: Allyn \& Bacon.

Davis, T., Johnson, K.R., \& Martinez, G.A. (2001). A reader on race, civil rights, and American law: A multiracial approach. Durham, NC: Carolina Academic Press.

DeCuir, J., \& Dixson, A. (2004). So when it comes out, they aren't that surprised that it is there: Using critical race theory as a tool of analysis of race and racism in education. Educational Researcher, 33(5), 26-31.

DeCuir-Gunby, J.T. (2006). Proving your skin is white, you can have everything: Race, racial identity, and property rights in whiteness in the Supreme Court case of Josephine DeCuir. In A. D. Dixson \& C.K. Rousseau (Eds.), Critical race theory in education: All God's children got a song (pp. 89-112). New York: Routledge.

Delgado, R., \& Stefancic, J. (2000). Critical race theory: The cutting edge ( $2^{\text {nd }}$ ed.). Philadelphia: Temple University Press.

Denzin, N.K., \& Lincoln, Y.S. (2000). Handbook of qualitative research. $\left(2^{\text {nd }}\right.$ ed.). Thousand Oaks, CA: Sage Publications. 
Denzin, N.K., \& Lincoln, Y.S. (2008). The discipline and practice of qualitative research. In N.K. Denzin \& Y.S. Lincoln (Eds.), The landscape of qualitative research (pp. 1-44). Thousand Oaks, CA: Sage Publications.

Dooley, E.A., \& Dooley, K.L. (2002). Legal foundations of special education for African American Learners. In E.F. Obiakor \& B.A. Ford (Eds.), Creating successful learning environments for African American learners with exceptionalities (pp. 17-26). Thousand Oaks, CA: Corwin.

Donnor, J. K., \& Brown, A. L. (2011). The education of Black males in a 'post racial' world. Race Ethnicity and Education, 14(1), 1-5.

Donovan, M.S., \& Cross, C.T. (Eds.). (2002). Minority representation in special and gifted education. Washington, DC: National Academy Press.

Dowling, M. (2005). From Husserl to Van Manen: A review of different phenomenological approaches. International Journal of Nursing Studies, 44(1), 131-142.

Doyle, S. (2007). Member checking with older women: A framework for negotiating meaning. Healthcare for Women International, 28(1), 888-908.

Driscoll, M. P. (2005). Psychology of learning and instruction. Boston, MA: Allyn \& Bacon.

DuBois, W.E.B. (1986). Writings. New York: Literary Classics of the United States. Eccles, J. S., Lord, S., \& Midgley, C. (1991). What are we doing to early adolescents? The impact of educational contexts on early adolescents. American Journal of Education, 99(1), 521-542.

Eccles, J.S., Wingfield, A., \& Shiefele, U. (1998). Motivation to succeed. In W. Damon 
(Series Ed.) \& N. Eisenberg (Vol. Ed.), Handbook of child psychology: Vol. 3. Social, emotional, and personality development ( $5^{\text {th }}$ ed.), (pp. 1017-1095). New York: Wiley.

Education for All Global Monitoring Report (2009). Educational marginalization in national education plans: Reaching the marginalized. United Nations Educational, Scientific and Cultural Organization. Retrieved from https://unesdoc.enesco.org Elementary and Secondary Education Act of 1965. Amended, Title V, Part D, Subpart 6: 20 U.S.C. 7253 et seq. Washington, DC: U.S. Department of Education.

Elhoweris, H., Mutua, K., Alsheikh, N., \& Holloway, P. (2005). Effect of children’s ethnicity of teacher's referral and recommendation decisions in gifted and talented programs. Remedial and Special Education, 26(1), 25-31.

Escalada, M.M., \& Heong, K.L. (1997). Focus group discussion. In K.L. Heong \& M.M. Escalada (Eds.), Methods for research on farmer's knowledge, attitudes, and practices in pest management (pp. 1-9). Manilla: International Rice Research Institute.

Federal Policy for the Protection of Human Subjects, 45 C.F.R. § 46 (2001). Retrieved http://www.hhs.gov/ohrp/humansubjects/guidance/45cfr46.htm

Fisher, E. J. (2005). Black student achievement and the oppositional culture model. The Journal of Negro Education, 74(3), 201-209.

Ford, D.Y. (1992). Determinants of underachievement as perceived by gifted, above average, and average black students. Roeper Review, 14(3), 130-136.

Ford, D. Y. (1996). Reversing underachievement among gifted Black students: Promising practices and programs. New York: Teachers College Press. 
Ford, D.Y. (1998). The underrepresentation of minority students in gifted education: Problems promises in recruitment and retention. Journal of Special Education, 32(1), 4-14.

Ford, D.Y. (2006). Closing the achievement gap: How gifted education can help. Gifted Child Today, 29(4), 14-18.

Ford, D.Y. (2010). Underrepresentation of culturally different students in gifted education: Reflections about current problems and recommendations for the future. Gifted Child Today, 33(3), 31-35.

Ford, D.Y. (2011). Closing the achievement gap: Gifted education must join the battle. Gifted Child Today, 24(1), 31-34.

Ford, D.Y. (2012). Ensuring equity in gifted education: Suggestions for change (again). Gifted Child Today, 35(1), 74-75.

Ford, D.Y. (2013). Recruiting and retaining culturally different students in gifted education. Waco, TX: Prufrock Press Inc.

Ford, D.Y. (2014). Segregation and the underrepresentation of Blacks and Hispanics in gifted education: Social inequality and deficit paradigms. Roeper Review, 36(3), 143-154.

Ford, D.Y., Coleman, M.R., \& Davis, J.L. (2014). Racially, ethnically, and linguistically different gifted and talented students. Gifted Child Today, 37(3), 133-134.

Ford, D., Grantham, T., \& Bailey, D. (1999). Identifying giftedness among African American males: Recommendations for effective recruitment and retention. In V. 
C. Polite \& J. E. Davis (Eds.), African American males in school and society: Practices and policies for effective education (pp. 51-67). New York: Teachers College Press.

Ford, D.Y., Harris, J.J., III, Tyson, C.A., \& Fraiser Trotman, M. (2002). Beyond deficit thinking: Providing access for gifted African America students. Roeper Review, 24(1), 52-58.

Ford, D. Y., \& Grantham, T. C. (2003). Providing access for culturally diverse gifted students: From deficit to dynamic thinking. Theory Into Practice, 4(1)2, 217-225.

Ford, D.Y., Grantham, T.C., \& Whiting, G.W. (2008). Culturally and linguistically diverse students in gifted education: Recruitment and retention issues. Exceptional Child, 74(3), 289-306.

Ford, D.Y., \& Helm, J.E. (2012). Testing and assessing African Americans: Unbiased tests are still unfair. The Journal of Negro Education, 81(3), 186-189.

Ford, D.Y., \& King, R.A. (2014). No Blacks allowed: Segregated gifted education in the context of brown v. board of education. The Journal of Negro Education, 83(3), $300-310$

Ford, D.Y., \& Moore, J.L. (2004). The achievement gap and gifted students of color. Understanding our Gifted, 16(1), 3-7.

Ford, D.Y., \& Moore, J.L. (2013). Understanding and reversing underachievement, low achievement, and achievement gaps among high-ability African American males in urban school contexts. Urban Review, 45, 399-415.

Ford, D. Y., Moore, J. L., III, \& Milner, H. R. (2005). Beyond cultureblindness: A model of culture with implications for gifted education. Roeper Review, 27(1), 97-103. 
Ford, D.Y., Moore, J.L., \& Trotman Scott., M. (2011). Key theories and frameworks for improving the recruitment and retention of African American students in gifted education. The Journal of Negro Education, 80(3), 239-253.

Ford, D.Y., \& Whiting, G.W. (2010). Beyond considerations in recruiting and retaining Gifted Black students. Journal for the Education of the Gifted, 34(1), 131-155.

Fossey, E., Harvey, C., McDermott, F. \& Davidson, L. (2002). Understanding and evaluating qualitative research. Australian and New Zealand Journal of Psychiatry, (36)1, 717-732.

Franklin, V.P. (2007). The tests are written for the dogs: African American children and the intelligence testing movement in historical perspective. The Journal of Negro Education, 76(1), 216-230.

Franklin, A. J., \& Boyd-Franklin, N. (2000). Invisibility syndrome: A clinical model of the effects of racism on African-American males. American Journal of Orthopsychiatry, 70(1), 33-41.

Freeman, M. (2008). Hermeneutics. In L.M. Given (Ed), Sage encyclopedia of qualitative research methods (pp. 385-388). Los Angeles, London, New Dehli, Singapore: Sage Publications.

Fryer, R.G., \& Torelli, P. (2010). An empirical analysis of acting White. Journal of Public Economics, 5(6), 380-396.

Furrer, C., \& Skinner, E. (2003). Sense of relatedness as a factor in children's academic engagement and performance. Journal of Educational Psychology, 95(1), 148 162.

Galbraith. J. (1985). The eight great gripes of gifted kids: Responding to special needs. 
Roeper Review. 8(1), 15-18.

Gall, M., Gall, J., \& Borg, W. (2007). Educational research: an introduction. New York, NY: Pearson.

Gantz, J. (2004). Fifty years after Brown: Tarnished gold; broken promises. The History Teacher, 38(1), 66-113.

García-Coll, C.T., Lamberty, G., Jenkins, R., McAdoo, H.P., Crnic, K., Wasik, B.H., \& Garcia, H.V. (1996). An integrative model for the study of the developmental capacities in minority children. Child Developmental, 67(5), 1891-1914.

Gearing, R. (2004). Bracketing in research a typology. Qualitative Health Research, 14(10), 1429-1452.

Gee, J.P. (2011). How to do discourse analysis: A toolkit. New York: Routledge.

Gentry, M., \& Springer, P M. (2002). Secondary student perceptions of their class activities regarding meaningfulness, challenge, choice, and appeal: An initial validation study. Journal Of Secondary Gifted Education, 13(1), 192-204.

Gering, R. (2004). Bracketing in research: A typology. Qualitative Health Research, 14(10), 1429-1452.

Gershenson, S., Holt, S.B., \& Papageorge, N.W. (2015). Who believes in me? The effect of student-teacher demographic match on teacher expectations (Upjohn Institute Working Paper 15-231). Kalamazoo, MI: W.E. Upjohn Institute for Employment Research.

Gibbs, G.R. (2002). Qualitative Data Analysis: Exploration with NVivo. Open University Press, Buckingham.

Gibson, W.J., \& Brown, A. (2009). Working with qualitative data. London: Sage. 
Gill, P., Stewart, K, Treasure, E., \& Chadwick, B. (2008). Methods of data collection in qualitative research: Interviews and focus groups. British Dental Journal, 204(6), 291-295.

Gillborn, D. (2015). Intersectionality, critical race theory, and the primacy of racism: Race, class, gender, and disability in education. Qualitative Inquiry, 23(3), 277287.

Giorgi, A. (2009). The descriptive phenomenological method in psychology: A modified Husserlian approach. Pittsburgh, PA: Duquesne University Press.

Glaser, B.G. (1992). Emerging vs. forcing: Basics of grounded theory analysis. Mills Valley, CA: Sociology Press.

Glesne, C. (2016). Becoming qualitative researchers: An introduction ( $5^{\text {th }}$ Ed.). Boston, MA: Pearson.

Glesne, C. \& Peshkin, A. (1992). Becoming qualitative researchers: An introduction. White Plains, NY: Longman.

Goldberg, B.M. (2013). White teachers in urban classrooms: Embracing students' nonWhite cultural capital for better teaching and learning. Urban Education, 49(1), $111-144$.

Gooden, A.S., \& McMahon, S.D. (2016). Thriving among African-American adolescents: Religiosity, religious support, and communalism. American Journal of Community Psychology, 57(1), 118-128.

Götz, T., Preckel, E, Pekrun, R., \& Hall, N. (2007). Emotional experiences during test taking: Does cognitive ability make a difference? Learning and Individual Differences, 17(1), 3-16. 
Graham, A., \& Anderson, K.A. (2008). "I have to be three steps ahead”: Academically gifted African American male students in an urban high school on the tension between an ethnic and academic identity. Urban Review, 40(1), 472-499.

Graneheim, U.H., \& Lundman, B. (2004). Qualitative content analysis in nursing research: Concepts, procedures and measures to achieve trustworthiness. Nurse Education Today, 24(1), 105-112.

Grbich, C. (2013). Qualitative Data Analysis: An Introduction (2 ${ }^{\text {nd }}$ ed.). London: Sage. Green, J., Draper, A.K. \& Dowler, E. (2003) Short cuts to safety: risk and 'rules of thumb' in accounts of food choice. Health, Risk and Society. 5(1), 33-52.

Gregory, A., \& Ripski, M. B. (2008). Adolescent trust in teachers: Implications for behavior in the high school classroom. School Psychology Review, 37(3), 337353.

Gregory, A., Skiba, R. J., \& Noguera, P. A. (2010). The achievement gap and the discipline gap: Two sides of the same coin? Educational Researcher, 39(1), 5968.

Grissom, J.A., Kalogrides, D., \& Loeb, S. (2015). Using student test scores to measure principal performance. Educational Evaluation and Policy Analysis, (37)1, 3-28.

Grissom, J. A., Nicholson-Crotty, J., \& Nicholson-Crotty, S. (2009). Race, region, and representative bureaucracy. Public Administration Review, 69(5), 911-919

Grissom, J.A., \& Redding, C. (2016). Discretion and disproportionality: Explaining the underrepresentation of high-achieving students of color in gifted programs. AERA Open, 2(1), 1-25.

Guinier, L., \& Torres, G. (2002). The miner’s canary: Enlisting race, resting power, 
transforming democracy. Cambridge, MA: Harvard University Press.

Guschker, S. (2000). Picture world and reality of life: A sociological study of the role of private photos for the meaningfulness of identity (unpublished doctoral thesis). Germany.

Hall, L., Jones, S., Hall, M., Richardson, J., \& Hodgson (2007). Inspiring design: The use of photo elicitation and lomography in gaining the child's perspective. Paper presented at Proceedings of the $21^{\text {st }}$ British HCI Group Annual Conference, University of Lancaster, United Kingdom.

Hardre, P. L., \& Reeve, J. (2003). A motivational model of rural students' intentions to persist in, versus drop out of, high school. Journal of Educational Psychology, 95(1), 347-356.

Hargrove, B.H., \& Seay, S.E. (2011). School teacher perceptions of barriers that limit the participation of African American males in public school gifted programs. Journal for the Education of the Gifted, 34(3), 434-467.

Harper, D. (2002). Talking about pictures: A case for photo elicitation. Visual Studies, 17(1), 13-26.

Harris, C. 1993. Whiteness as property. Harvard Law Review 106(8), 1707-1791.

Harris, J. J., III, Brown, E. L., Ford, D. Y., \& Richardson, J. W. (2003). African Americans and multicultural education: A proposed remedy for disproportionate special education placement and underinclusion in gifted education. Education and Urban Society, 36(3), 304-341.

Harmon, D. (2002). They won't teach me: The voices of gifted African American inner- 
city students: Improving racial identity improves achievement. Roeper Review, 24(1), 68-75.

Harvey, W. B. (2008). The weakest link: A commentary on the connections between K12 and higher education. American Behavioral Scientist, 51(7), 972-983.

Hatch, J.A. (2002). Doing research in education settings. New Albany, NY: State University of New York Press.

Hébert, T.P. (2002). Gifted Black males in predominantly White university: Portraits of high achievement. Journal for the Education of the Gifted, 26(1), 25-64.

Hébert, T. P., \& Schreiber, C. (2010). An examination of selective achievement in gifted males. Journal for the Education of the Gifted, 33(1), 570-605.

Heidegger, M. (1962). Being and Time. New York: Harper and Row.

Helm, J.H. (2008). Got standards? Don’t give up on engaged learning. Retrieved from http://miracosta.edu/instruction/childdevelopmentcenter/downloads/6.5EngagedL earning.pdf

Helms, J. (1985). Toward a theoretical explanation of the effects of race on counseling: A Black and White model. The Counseling Psychologist, 12(1), 153-165.

Hemmings, A. (1996). Conflicting images? Being Black and a model high school student. Anthropology and Education Quarterly, 27(1), 20-50.

Henfield, M. S. (2013). Special issue: Meeting the needs of gifted and high-achieving Black males in urban schools. Urban Review, 45, 395-398.

Henfield, M.S., Moore, J.L., \& Wood, C. (2008). Inside and outside gifted education programming: Hidden challenges for African American students. Council for Exceptional Children, 74(4), 433-450. 
Henfield, M. S., D. Owens, \& J. L. Moore III. (2008). Influences on Young Gifted African Americans’ School Success: Implications for Elementary School Counselors. The Elementary School Journal, 108(1), 392-406.

Henfield, M.S., Washington, A.R., \& Byrd, J.A. (2014). Addressing academic and opportunity gaps impacting gifted black males: Implications for school counselors. Gifted Child Today, 37(3), 147-154.

Henfield, M.S., Washington, A.R., \& Owens, D. (2010). To be or not to be gifted: The choice for a new generation. Gifted Child Today, 32(2), 17-25.

Henfield, M.S., Woo, H., \& Bang, N. (2017). Gifted ethnic minority students and academic achievement: A meta-analysis. Gifted Child Quarterly, 61(1), 3-19.

Hertberg-Davis, H. (2009). Myth 7: Differentiation in the regular classroom is equivalent to gifted programs is equivalent. Gifted Child Quarterly, 53(4), 251-253.

Hertzog, N. B. (2003). Impact of gifted programs from the students’ perspectives. Gifted Child Quarterly, 47, 131-143.

Hilliard, A. (2003). No mystery: Closing the achievement gap between Africans and excellence. In T. Perry, C. Steele, \& A. Hilliard (Eds.), Young, gifted and black: Promoting high achievement among African-American students (pp. 131-165). Boston: Beacon Press.

Hoepfl, M. (1997). Choosing qualitative research: a primer for technology education researchers. Journal of Technology Education, 9(1), 48-59.

Holzer, H.M. (2006, May 15). Reconnecting your Black men. The Washington Post. Retrieved from http://www.washingtonpost.com 
Hopkins, R. (1997). Educating Black males: Critical lessons in schooling, community and power. Albany: State University of New York Press.

Howard, T. C. (2008). Who really cares? The disenfranchisement of African American males in prek-12schools: A critical race theory perspective. Teacher's College Record, 110(5), 954-985.

Howard, T. C. (2010). Why race and culture matter in schools: Closing the achievement gap in America's classrooms. New York: Teachers College Press.

Howard, T. C. (2013). How does it feel to be a problem? Black male students, schools, and learning in enhancing the knowledge base to disrupt deficit frameworks. Review of Research in Education, 37(1), 54-86.

Howard, T. C., \& Flennaugh, T. (2011). Research concerns, cautions, \& considerations on Black males in a "post-racial” society. Race, Ethnicity \& Education, 14(1), 105-120.

Howard, T.C., Flennaugh, T.K., \& Terry, C.L. (2012). Black males, social imagery, and the disruption of pathological identities: Implications for research and teaching. Educational Foundations, 26(1), 85-102.

Hseih, P., Sullivan, J.R., \& Guerra, N.S. (2007). A closer look at college students: Efficacy and goal orientation. Journal of Advanced Academics, 18, 454-476.

Hyland, N.E. (2005). Being a good teacher of Black students? White teachers and unintentional racism. Curriculum Inquiry, 35(1), 429-459.

Janesick, V.J. (2013). Oral History, Life History, and Biography. In A.A. Trainor \& E. Graue (Ed.), Reviewing Qualitative Research in the Social Sciences (pp. 151165). New York, NY: Routledge. 
Jacob K. Javits Gifted and Talented Students Education Program. (1988). Program details. Retrieved from http://www2.ed.gov/programs/javits/index.html

Jenkins, K.N., Woodard, R., \& Winter, T. (2008). The emergent production of analysis in photo elicitation: Pictures of military identity. Forum: Qualitative Research, 9(3), 49-61.

Irvine, J.J. (1985). Teacher communication patterns as related to the race and sex of the student. Journal of Educational Research, 78(1), 338-345.

Jick, T.D. (1979). Mixing qualitative and quantitative methods: Triangulation in action. Administrative Science Quarterly, 24(1), 602-611.

Jolly, J.L. (2009). The national defense act, current stem initiative, and the gifted. Gifted Child Today, 32(2), 50-53.

Jonson-Reid, M., Davis, L., Saunders, J., Williams, T., \& Williams, J.H. (2005). Academic self-efficacy among African American youths: Implications for school social work practice. Children and School, 10(1), 5-14.

Kamins, M.L., \& Dweck, C.S. (1999). Person versus process praise and criticism: Implications for contingent self-worth and coping. Developmental Psychology, 35(3), 835-847.

Kearns, T., Ford, L., \& Linney, J. A. (2005). African American student representation in special education programs. The Journal of Negro Education, 74(1), 297-310

Kentucky Department of Education, School Report Card. (2016). Retrieved from http:// education.ky.gov/AA/distsupp/Pages/SRC.aspx

Kerpelman, J.L., Eryigit, S., \& Stephens, C.J. (2008). African American adolescents’ 
future education orientation: Associations with self-efficacy, ethnic identity, and perceived parental support. Journal of Youth and Adolescence, 37(1), 997-1008.

Kitzinger, J. (1995). Introducing focus groups. BMJ, 311(1), 299-302.

Klinger, J.K., Cramer, E., \& Harry, B. (2006). Challenges in the implementation of Success for All by four urban schools. Elementary School Journal, 106(1), 333349.

Koch, T. (1995). Interpretive approaches in nursing research: The influence of Husserl and Heidegger. Journal of Advanced Nursing, 21(5), 827-836.

Kretsedemas, P. (1993). Reading advertisements: race vs. ethnicity?. Visual Sociology, $8(2), 40-47$.

Krueger, R.A. (1994). Focus groups: A practical guide for applied research. Thousand Oaks, CA: Sage Publications.

Krueger, R \& Casey, M.A. (2000). Chapter 1: Overview of focus groups. In: Focus Groups (p. 3-19). Thousand Oaks, CA: Sage Publications.

Kumar K (1987) Conducting group interviews in developing countries. AID program design and evaluation methodology report no. 8. Agency for International Development, Washington D.C.

Ladson-Billings, G., \& Tate, W.F. (1995). Toward a critical race theory of education. Teachers College Record, 97(1), 47-58.

Ladson-Billings, G., \& Tate, W.F. (2006). Toward a critical race theory of education. In A. D. Dixson \& C.K. Rousseau (Eds.), Critical race theory in education: All God's children got a song (pp. 89-112). New York: Routledge.

Lederman, N. (1995). Translation and transformation of teachers' understanding of the 
nature of science into classroom practice. Science Education, 71(1), 721-734.

Leedy, P., \& Ormrod, J. (2001). Practical research: Planning and design (7 ${ }^{\text {th }}$ ed.). Upper Saddle River, NJ: Merrill Prentice Hall. Thousand Oaks: SAGE Publications.

Lee, S.Y., Olszweski-Kubilius, P., \& Thomson, D. (2012). Academically gifted students’ perceived interpersonal competence and peer relationships. Gifted Child Quarterly, 56(2), 90-104.

LeVasseur, J. (2003). The problem of bracketing in phenomenology. Qualitative Health Research, 13(3), 408-420.

Lewis, A. E. (2003). Race in the schoolyard: Negotiating the color line in classrooms and communities. New Brunswick, NJ: Rutgers University Press

Lim, H. (2006). Representative bureaucracy: Rethinking substantive effects and active representation. Public Administration Review, 66(1), 193-204.

Lincoln, Y., \& Guba, E. (1985) Naturalistic inquiry. Newbury Park, CA: Sage Publications.

Lohman, D.F., \& Hagen, E.P. (2005). Cognitive abilities test form 6. Gifted Child Quarterly, 13(2), 18-27.

Lopez, K.A., \& Willis, D.G. (2004). Descriptive versus interpretive phenomenology: Their contributions to nursing knowledge. Qualitative Health Research, (14)5, 726-735.

Louie, J. (2005). We don't feel welcome here: African Americans and Hispanics in Metro. Cambridge, MA: The Civil Rights Project at Harvard University. Louis, B., Subotnik, R.F., Breland, P.S., \& Lewis, M. (2000). Establishing criteria for 
high ability versus selective admission to gifted programs: Implications for policy and practice. Educational Psychology Review, 12(3), 295-314.

Lynn, M., Bacon, J. N., Totten, T. L., Bridges III, T. L., \& Jennings, M. (2010). Examining 80 teachers' beliefs about African American male students in a lowperforming high school in an African American school district. Teachers College Record, 112(1), 289-330.

Lynn, M., \& Parker, L. (2006). Critical race studies in education: Examining a decade of research on U.S. school. The Urban Review, 38(4), 257-290.

Mack, N., Woodsong, C., Macqueen, KM., Guest, G., \& Namey, E., (2005). Qualitative research methods: A data collector's field guide. Research Triangle Park, NC: Family Health International.

Maggs-Rapport, F. (2000). Combining methodological approaches in research: Ethnography and interpretive phenomenology. Journal of Advanced Nursing, 31(1), 219-225.

Majors, R., \& Billson, J. M. (1992). Cool pose: The dilemmas of Black manhood in America. New York: Lexington Books.

Marland, S., Jr. (1972). Education of the gifted and talented: Report to the Congress of the United States by the U.S. Commissioner of Education. Washington, DC: Washington Press.

Marshall, C., \& Rossman, B. R. (2006). Designing qualitative research. (4th ed.), Thousand Oaks, CA: Sage Publication, Inc.

Mason, J. (2013). Qualitative Researching ( $2^{\text {nd }}$ ed.). Sage Publications, London. Mateu-Gelabert, P., \& Lune, H. (2007). Street codes in high school: School as an 
educational deterrent. City and Community, 6(1), 173-191.

Matthews, M. S., \& Kirsch, L. (2011). Evaluating gifted identification practice: Aptitude testing and linguistically diverse learners. Journal of Applied School Psychology, 27(1), 155-180.

McBee, M.T. (2006). A descriptive analysis of referral sources for gifted identification screening by race and socioeconomic status. Journal of Secondary Gifted Education, 17(1), 103-111. Government Printing Office. Retrieved from http://www.files.eric.edu.gov

McBee, M. (2010). Examining the probability of identification for gifted programs for students in Georgia elementary Schools: A multilevel path analysis study. Gifted Child Quarterly, 54(4), 283-297.

McClain, M., \& Pfeiffer, S. (2012). Identification of gifted students in the United States today: A look at state definitions, policies and practice. Journal of Applied School Psychology, 28(1), 59-88.

McClelland, D. C. (1961). The achieving society. Princeton, NJ: Van Nostrand.

McGhee, E.O., (2013). Threatened and placed at risk: High achieving African American males in urban high schools. Urban Review, 45(4), 448-471.

McIntush, H.G. (2000). The rhetorical enactment of ideology in “a nation at risk”. Rhetoric \& Public Affairs, 3(3), 419-443.

McRoy, R. (1996). Qualitative Research. Retrieved from http://www.uncp.edu/home/marson/qualitative_research.html Mendoza-Denton R, Downey G, Purdie V, Davis A, Pietrzak J. (2002). Sensitivity to 
status-based rejection: Implications for African-American college students’ college experience. Journal of Personality and Social Psychology, 83(4), 896918.

Merriam, S. B. (2002). Assessing and evaluating qualitative research. In S.B. Merriam (Ed.), Qualitative research in practice: Examples for discussion and analysis (pp.18-33). San Francisco: Jossey Bass.

Michael-Chadwell, S. (2010). Examining the underrepresentation of underserved students in gifted programs from a transitional leadership vantage point. Journal for the Education of the Gifted, (34)1, 99-130.

Mickelson, R.A. (1990). The attitude-achievement paradox. Sociology of Education, 63(1), 44-61.

Mickelson, R.A. (2003). When are racial disparities in education the result of racial discrimination? A social science perspective. Teacher's College Record, 105(6), 1052-1086.

Mickelson, R.A., \& Greene, A.D. (2006). Connecting pieces of the puzzle: Gender differences in Black middle school students' achievement. The Journal of Negro Education, 75(1), 34-48,

Miles, M.B., Huberman, A.M., \& Saldaña, J. (2014). Fundamentals of qualitative data analysis. In Qualitative data analysis: A methods sourcebook (pp. 69-106). Thousand Oaks, CA: Sage Publications.

Milner, H.R. (2007). African-American males in urban schools: No excuses-teach and empower. Theory Into Practice, 46(3), 239-246.

Milner, H.R. (2010). Start where you are, but don't stay there: Understanding diversity, 
opportunity gaps, and teaching in today's classrooms. Cambridge, MA: Harvard Education Press.

Mitchell, C. (2011). Doing visual research. Thousand Oaks, CA: SAGE Publications. Moon, T.R., Tomlinson, C.A., \& Callahan, C.M. (1995). Academic diversity in the middle school: Results of a national survey of middle school administrators and teachers (Research Monograph 95124). Storrs, CT: National Research Center on the Gifted and Talented, University of Connecticut.

Moon, T.R., Brighton, C.M., \& Callahan, C.M. (2003). State standardizing testing program: Friend or foe of gifted education? Roeper Review, 25(1), 49-60.

Moore, J.L., Ford, D.Y., \& Milner, H.R. (2005). Recruitment is not enough: Retaining African American students in gifted programs. Gifted Child Quarterly, 49(1), 5167.

Moore, J.L., \& Owens, D. (2008). Educating and counseling African- American students: Recommendations for teachers and school counselors. In L. Tillman \& G. Cartledge (Eds.), Educating African Americans (pp. 351-366). Thousand Oaks, CA: Sage.

Morse, J. M. (1994). Designing funded qualitative research. In Denizin, N. K. \& Lincoln, Y.S., Handbook of qualitative research (2nd Ed). Thousand Oaks, CA: Sage. Morse, J. M., Barrett, M., Mayan, M., Olson, K., \& Spiers, J. (2002). Verification strategies for establishing reliability and validity in qualitative research. International Journal of Qualitative Methods, 1(2), 1-19.

Moustakas, C. (1994). Phenomenological research methods. Thousand Oaks, CA: Sage. Myrdal, G. (1964). An American dilemma. New York, NY: McGraw-Hill. 
Naglieri, J. A., \& Ford, D. (2005). Increasing minority children's participation in gifted classes using the NNAT: A response to Lohman. Gifted Child Quarterly, 49(1), $29-36$.

Natanson, M. (1973). Edmund Husserl: Philosophy of infinite tasks. Evanston, IL: Northwestern University Press.

National Association for Gifted Children. (2008). Why we should advocate for gifted and talented students. Retrieved from https://www.nagc.org/resources- publications National Association for Gifted Children, \& Council of State Directors of Program for the Gifted. (2009). State of the states in gifted education, 2008-2009, summary of findings. Washington, DC: National Association for Gifted Children.

National Association for Gifted Children, The Council of State Directors of Programs for the Gifted. (2015). 2014-2015 State of the states in gifted education. Retrieved from National Association for Gifted Children http://www.nacg.org

National Center for Education Statistics. (2006). [Digest of Education Statistics: 2006, Table 51]. Number and percentage of gifted and talented students in public elementary and secondary schools by sex, race/ethnicity, and state:2002. Retrieved from http://nces.ed.gov/programs/digestd06/tables/dt06_051.asp. National Center for Education Statistics. (2009). [Digest of Education Statistics: 2006, Table 54].Percentage of gifted and talented students in public elementary and secondary schools, by sex, race/ethnicity, and state: 2004 and 2006. Retrieved from http:// files.eric.ed.gov/fulltext/ED504502.pdf

National Center for Education Statistics. (2011). The condition of education 2011: Participation in education. Retrieved from https:// 
nces.ed.gov/pubs2011/2011033_2.pdf

National Center for Education Statistics. (2015). Number of public school students enrolled in gifted and talented programs, by sex, race/ethnicity, and state: 2004, 2006, and 2011-12 (Digest of Education Statistics). Retrieved from htpps://www.nces.ed.gov/pubs2016/2016014.pdf

National Commission on Excellence in Education. (1983). A nation at risk. Retrieved from http://www.ed.gov/pub/NatAtRisk/index.html

No Child Left Behind Act [NCLB]. (2002). P.L. 107-110. Retrieved from http:// www.nochildleftbehind.gov/

Neblett, E. W., Jr., Philip, C. L., Cogburn, C. D., \& Sellers, R. M. (2006). African American adolescents' discrimination experiences and academic achievement: Racial socialization as a cultural compensatory and protective factor. Journal of Black Psychology, 32(1), 199-218.

Neihart, M. (2007). The socio-affective impact of acceleration and ability grouping: Recommendation for best practice. Gifted Child Quarterly, 51, 330-341.

Nicholson-Crotty, J., Grissom, J.A., \& Nicholson-Crotty, S. (2011). Bureaucratic representation, distributional equity, and democratic values in the administration of public programs. Journal of Politics, 73(2), 582-596.

Noble, R. (2011). Mathematics self-efficacy and African American male students: An examination of models of success. Journal of African American Males in Education, 2(2), 188-213.

Noble, K. D., Robinson, N. M., \& Gunderson, S. A. (1993). All rivers lead to the sea: A follow-up study of young adults. Roeper Review, 15(1), 124-129. 
Noble, R. (2011). Mathematics self-efficacy and African American male students: An examination of models of success. Journal of African American Males in Education, 2(2), 188-213.

Noble, H., \& Smith, J. (2015). Issues of validity and reliability in qualitative research. Evidence Based Nursing, 18, (2), 34-35.

Noguera, P. (2008). The trouble with black boys and other reflections on race, equity and the future of public education. San Francisco, CA: John Wiley and Sons.

Noguera, P. (2009). The trouble with black boys and other reflections on race, equity, and the future of public education. San Francisco, CA: Jossey Bass.

No Child Left Behind Act of 2001 (NCLB). 2001. Public Law 107-110 107th Congress. Title IX, Part A, Sec. 9101(22), Subpart 6-Gifted and talented students. Washington, DC: U.S. Department of Education. Retrieved from https//:www.congress.gov/bill/107 ${ }^{\text {th }}$-congress/house-bill/1

Oakes, J. (2005). Keeping track: How schools structure inequality ( ${ }^{\text {nd }}$ ed.). London: Yale University Press.

Ogbu, J.U. (1992) Understanding cultural differences and school learning. Education Libraries, 16(4), 7-11.

Ogbu, J.U. (2004). Collective identity and the burden of "acting White” in Black history, community, and education. The Urban Review, 36(1), 1-35.

Ogbu, J.U., \& Simmons, H.D (1998). Voluntary and involuntary minorities: A culturalecological theory of school performance and some implications for education. Anthropology \& Education, 29(1), 155-188.

Olivos, E.M. (2006). Racism and deficit thinking. Counterpoints, 209(1), 41-59. 
Olszewski-Kubilius, P., \& Clarenbach, J. (2014). Closing the opportunity gap: Program factors contributing to academic success in culturally different youth. Gifted Child Today, 37(1), 103-110.

Olszewski-Kubilius, P., \& Thomson, D.L. (2010). Gifted programming for poor and minority urban students: Issues and lessons learned. Gifted Child Today, 33(4), 58-64.

Osterman, K. F. (2000). Students' need for belonging in the school community. Review of Educational Research, 70(1), 323-367.

Oxford Illustrated Dictionary (1998). New York, NY: DK Publishing Inc.

Pace, J. L., \& Hemmings, A. (2007). Understanding authority in classrooms: A review of theory, ideology, and research. Review of Educational Research, 77(1), 4-27.

Pajares, F., \& Miller, M. D. (1994). Role of self-efficacy and self-concept beliefs in mathematical problem solving: A path analysis. Journal of Educational Psychology, (86)1, 193-203.

Pajares, F. (2002). Gender differences in mathematics: An integrative psychological approach. In A.M. Gallager \& J.C. Kaufman (Eds.), Gender differences in mathematics self-efficacy beliefs (pp. 294-315). Cambridge, NY: Cambridge University Press.

Palmer, R.T., Davis, R.J., \& Hilton, A.A. (2009). Exploring challenges that threaten to impede the academic success of academically underprepared Black males at an HBCU. Journal of College Student Development, 50(4), 429-445.

Palmer, R. T., Maramba, D. C., \& Dancy, T. E. (2011). A qualitative investigation of 
factors promoting the retention and persistence of students of color in STEM. Journal of Negro Education, 80(4), 491-504.

Parham, T.A. (1989). Cycles of psychological nigrescence. The Counseling Psychologist, 17(1), 187-226.

Parham, T.A., \& Helms, J.E. (1985). Relation of racial identity attitudes to selfactualization and effective states of Black students. Journal of Counseling Psychology, 32(1), 431-440.

Parker, L. (2008). Critical race theory and Africana studies: Making connections to education. Cave Hill. University of Wisconsin.

Parker, L., \& Lopez, G. (2003). Interrogating racism in qualitative research methodology. Counterpoints. New York, NY: Peter Lang Publishing.

Patrick, H., Gentry, M., \& Owen, S. V. (2006). Motivation and gifted adolescents. In F. Dixon \& S. M. Moon (Eds.), The handbook of secondary gifted education (pp. 165-195). Waco, TX: Prufrock Press.

Patton, M. Q. (2002). Qualitative research \& evaluation methods (2nd ed.), Thousand Oaks, CA: Sage Publications, Inc.

Payne, A. (2011). Payne, A. (2011). Equitable access for underrepresented students in gifted education. Retrieved from http://files.eric.ed.gov/fulltext/ED539772.pdf

Petty, N.J, Thomson, O.P., \& Stew, G. (2012). Ready for a paradigm shift? Part 2: Introducing qualitative research methodologies and methods. Manual Therapy, (17)5, 378-384.

Pfeiffer, K. (2003). Race passing and American individualism. Boston, MA: University of Massachusetts Press.

Phinney, J.S. (1990). Ethnic identity in adolescents and adults: Review of research. 
Psychological Bulletin, 108(1), 499-514.

Pietkiewicz, I., \& Smith, J.A. (2014). A practical guide to using interpretative phenomenological analysis in qualitative research psychology. Czasopismo Psychologiczne-Psychological Journal, 20(1), 7-14.

Piirto, J. (1999). Talented adults and children: Their development and education (2nd ed.). Englewood Cliffs, NJ: Prentice Hall.

Platt, L. (2002). New jack jocks: Rebels, race, and the American athlete. Philadelphia: Temple University Press.

Plunker, J. A., Burroughs, N., \& Song, R. (2010). Mind the (other) gap! The growing excellence gap in K-12 education. Bloomington, IN: Center for Evaluation and Education Policy.

Plucker, A., Robinson, N. M., Greenspon, T. S., Feldhusen, F., McCoach, D. H., \& Subotnik, R. F. (2004). It's not how the pond makes you feel, but rather how high you can jump. American Psychologist, 59(1), 268-269.

Polite, V.C. (1993). If only we knew then what we know now: Foiled opportunities to learn in suburbia. Journal of Negro Education, 62(1), 337-354.

Polite, V.C. (1994). The method in the madness: African American males, avoidance schooling chaos theory. Journal of Negro Education, 63(1), 588-601.

Polit, D.F., \& Hungler, B.P. (1999). Principles and Methods (6 ${ }^{\text {th }}$ ed.). Philadelphia, PA: Lippincott Company.

Popay, J., Rogers, A., \& Williams, G. (1998). Rationale and standards for the systematic review of qualitative literature in health services research. Qualitative Health Research, (8)1, 341-351. 
Preckel, F., Götz, T., \& Frenzel, A, (2010). Ability grouping of gifted students: Effects on academic self-concept and boredom. British Journal of Educational Psychology, (80)1, 451-472.

Punch, S. (2009). Case study: Researching childhoods in Bolivia. In E.K.M. Tisdall, J.M. Davis, \& M. Gallagher (Eds.), Researching with children and young people (pp. 89-96). London: Sage.

Rabiee, F., (2004). Focus-group interview and data analysis. Proceedings of the Nutrition Society, UK, 63, 655-660. doi:10.1079/PNS2004399

Rager, K. B. (2005). Self-care and the qualitative researcher: when collecting data can break your heart. Educational Researcher, 34(4), 23-27.

Romanoff, B.S., Algozzine, B., \& Neilson, A.B. (2009). Achievement of African American and White students referred and placed or not placed in gifted programs. Journal for the Education of the Gifted, 33(2), 156-175.

Reiners, G.M. (2012). Understanding the differences between Husserl's (descriptive) and Heidegger’s (interpretive) phenomenological research. Nursing and Care, 1(5), 37-49.

Reis, S.M., Gubbins, E.J., Briggs, C., Schreiber, F.R., Richards, S., \& Jacobs, J. (2004). Reading instruction for talented readers: Case studies documenting few opportunities for continuous progress. Gifted Child Quarterly, 48(1), 309-338.

Reiter, S., Stewart, G., \& Bruce, C. (2011). A strategy for delayed research method selection: Deciding between grounded theory and phenomenology. Electronic Journal of Business Research Methods, 9(1), 35-46.

Resnick, M. D., Bearman, P. S., Blum, R. W., Bauman, K. E., Harris, K. M., Jones, J., \& 
Udry, D. (1997). Protecting adolescents from harm: Findings from the National Longitudinal Study on Adolescent Health. JAMA, 27(1), 823-832.

Resnick, L., Levine, J.M., \& Teasley, S.D. (Eds.). (1991). Perspectives on socially shared cognition. Washington, DC: American Psychological Association.

Riphagen, L. (2008). Marginalization of African Americans in the social sphere of U.S. society. Interdisciplinary Journal of International Studies, 5(1), 95-121.

Ritchie, J., \& Spencer, L. (1994). Qualitative data analysis for applied policy research. In Analyzing qualitative data (pp. 173-194). London: Routledge.

Robinson, A. (2008). Teacher characteristics. In J.A. Plucker and C.M. Callahan (Eds.), Critical issues and practices in gifted education: What the research says (pp. 681694). Waco, TX: Prufrock Press.

Rocha, R.R., \& Hawes, D.P. (2009). Racial diversity, representative bureaucracy, and equity in multiracial school districts. Social Science Quarterly, 90(2), 326-344.

Roderick, M. (2003). What's happening to the boys? Early high school experiences and school outcomes among African American male adolescents in Chicago. Urban Education, 38(1), 538-607.

Rogers, K. B. (2007). Lessons learned about educating the gifted and talented: A synthesis of the research on educational practice. Gifted Child Quarterly, 51(1), 382-396.

Rogoff, B. (2003). The cultural nature of human development. New York: Oxford University Press.

Rojewski, J.W., \& Kim, H. (2003). Career choice patterns and behavior of work-bound youth during early adolescence. Journal of Career Development, 30(1), 89-108. 
Romanoff, B.S., Algozzine, B., \& Nielson, A.B. (2009). Achievement of African American and White students referred and placed or not placed in gifted programs. Journal for the Education of the Gifted, (33)2, 156-175.

Rothman, B. K. (1989). Motherhood under Capitalism. In Recreating Motherhood: Ideology and Technology in a Patriarchal Society (pp. 39-50). New York: Norton.

Rubin, H. J., \& Rubin, I. S. (2005). Qualitative interviewing: The art of hearing data. Thousand Oaks, CA: Sage Publications, Inc.

Sailes, G. A. (Ed.). (2003). African Americans in sports. Brunswick, NJ: Transaction. Saldaña, J. (2016). The coding manual for qualitative researchers. Thousand Oaks, CA: Sage Publications.

Sampson-Cordle, A.V. (2001). Exploring the relationship between a small rural school in Northeast Georgia and its community: An image based study using participantproduced photographs (unpublished doctoral thesis), Athens, GA.

Saunders, J., Davis, L., Williams, T., \& Williams, J.H. (2004). Gender differences in selfperceptions and academic outcomes: A study of African American high school students. Journal of Youth and Adolescence, 33(1), 81-90.

Schott Foundation for Public Education (2010). Yes we can: The 2010 Schott 50 state report on public education and Black males. Retrieved from http:// edexcellence.net/commentary/education-gadfly-weekly/2010/august-26/schottfoundation-yes-we-can-the-2010-schott-50-state-report-on-public-education-andblack-males.html

Schott Foundation for Public Education (2012). The urgency of now: The Schott 50 state 
report on public education and black males. Retrieved from http://schottfoundation.org/blog/2012/09/18/schott-50-state-report

Scott, M.T. (2012). Socio-emotional and psychological issues and needs of gifted African-American students: culture matters. Interdisciplinary Journal of Teaching and Learning, 2(1), 23-33.

Schwandt, T.A (2005). Three epistemological stances for qualitative inquiry. In: Denzin, N.K., Lincoln, Y.S. (eds.) Handbook of Qualitative Research, ( $3^{\text {rd }}$ ed.). Sage Publications, London.

Seaton, M., Marsh, H. W., \& Craven, R. G. (2009). Earning its place as a pan-human theory: Universality of the big-fish-little-pond effect across 41 culturally and economically diverse countries. Journal of Educational Psychology, 101, 404419. doi:10.1037/a0013838

Sellers, R., Copeland-Linder, N., Martin, P., \& Lewis, R. (2006). Racial identity matters: The relationship between racial discrimination and psychological functioning in African American adolescents. Journal of Research on Adolescence, 16(2), 187216.

Seigle, D., Wilson, H.E., \& Little, C.A. (2013). A sample of gifted and talented educators’ attitudes about academic acceleration. Journal of Advanced Academics, 24(1), 27-51.

Sethna, B. N., Wickstrum, C. D., Boothe, D., \& Stanley, J. C. (2001). The advanced academy of Georgia: Four years as a residential early-college entrance program. Journal of Secondary Gifted Education, 13(1), 11-22.

Shenton, A.K. (2004). Strategies for ensuring trustworthiness in qualitative projects. 
Education for Information, 22(1), 63-75.

Simmons D.C. (1998). What reading research tells us about children with diverse learning needs. Mahwah, NJ: Erlbaum.

Skiba, R.J., Michael, R.S., Nardo, A.C., Peterson, R.L. (2002). The color of discipline: Sources of racial and gender disproportionality in school punishment. Urban Review, 34(4), 317-342.

Skiba, R. J., Knesting, K., \& Bush, L. D. (2002). Culturally competent assessment: More than nonbiased tests. Journal of Child and Family Studies, 11(1), 61-78.

Smith, D.E. (1987). The everyday world as problematic: A feminist sociology. Boston: Northwestern University Press.

Smith, J.A., \& Osborn (2007). Interpretive phenomenological analysis. In John Smith (Ed.), Qualitative psychology: A practical guide to research methods (pp. 53-80). Thousand Oaks, CA: Sage Publications, Inc.

Smith, J. A., Flowers, P., \& Larkin, M. (2009). Interpretative phenomenological analysis: Theory, method, and research. London, England: Sage Publications.

Solokowski, R. (2000). Phenomenology defined. In Introduction to phenomenology (pp. 185-197). New York, NY: Cambridge University Press.

Solomon, J. L. (1987). From Hegel to existentialism. New York: Oxford University Press.

Solomon, R.P., Portelli, J.P., Daniel, B.J., \& Campbell, A. (2005). The discourse of denial: How white teacher candidates construct race, racism, and "white privilege”. Race, Ethnicity and Education, 8(1), 147-169.

Soloranzo, D.G. (1997). Images and Words that Wound: Critical Race Theory, Racial 
Stereotyping, and Teacher Education. Teacher Education Quarterly, 24(3), 5-19. Southern, W. T., \& Jones, E. D. (2004). Types of acceleration: Dimensions and issues. In N. Colangelo, S. Assouline, \& E. Gross (Eds.), A nation deceived: How schools hold back America’s brightest students (pp. 5-12). Iowa City: University of Iowa. Speirs Neumeister, K.L., Adams, C.M., Pierce, R.L., Cassady, J.C., \& Dixon, F.A. (2007). Fourth-grade teachers' perception of giftedness: Implications for identifying and serving diverse gifted students. Journal for the Education of the Gifted, 30(1), 479-499.

Spencer, M.B., \& Markstrom-Adams, C. (1990). Identity processes among racial and ethnic children in America. Child Development, 61(1), 290-310.

Spielgelberg, H. (1976). Doing phenomenology. Hague, Netherlands: Martinus Nijhoff. Staigner, A. (2004). Whiteness as giftedness: Racial formation at an urban high school. Social Problems, 51(2), 161-181.

Stambaugh, T., \& Ford, D. Y. (2015). Microaggressions, multiculturalism, and gifted individuals who are Black, Hispanic, or poor: Cultural and economic considerations for counseling. Journal of Counseling and Development, 93(1), 192-201.

Strauss, A.L. (1987). Qualitative analysis for social scientists. Cambridge: Cambridge University Press.

Strauss, A. \& Corbin, J. (1998). Basics of qualitative research: Grounded theory procedures and technique ( $2^{\text {nd }}$ Ed.). New Park, London: Sage.

Stewart, D., \& Mickunas, A. (1974). Exploring phenomenology: A guide to the field and its literature. Chicago, IL: American Library Association. 
Subotnik, R.F., Olszewski-Kubilius, P., \& Worrell, F.C. (2011). Rethinking giftedness and gifted education: A proposed direction forward based on psychological science. Psychological Science in the Public Interest, 12(1), 3-54.

Subotnik, R. F., Olszewski-Kubilius, P., \& Worrell, F. C. (2012). A proposed direction forward for gifted education based on psychological science. Gifted Child Quarterly, 56(1), 176-188.

Sue, D.W. (2010). Microaggressions in everyday life: Race, gender, and sexual orientation. Hoboken, NJ: Wiley \& Sons, Inc.

Swanson, J. D. (2007). Policy and practice: A case study of gifted education policy implementation. Journal for the Education of the Gifted, 31(1), 131-164.

Tate, W. (1997). Critical race theory and education: History, theory, and implications. Review of Research in Education, 22(1), 195-247.

Tatum, A. (2005). Teaching reading to black adolescent males: Closing the achievement gap. New York: Stenhouse.

Thomas, K. (2009). Evaluating psychological factors that relate to academic success among high-achieving African American males. An Addendum to Leading Change in Gifted Education, 1(1), 20-24.

Tomlinson, C.A. (2003). Fulfilling the promise of the differentiated classroom: Strategies and tools for responsive teaching. Alexandria, VA: Association for Supervision and Curriculum Development.

Tomlinson, C.A., \& Jarvis, J.M. (2014). Supporting academic success for students with high 
potential from ethnic minority and economically disadvantaged backgrounds. Journal for the Education of the Gifted, 37(3), 191-219.

Trautwein, U., Lidtke, O., Marsh, H.W., \& Nagy, G. (2008). Within school comparisons: Perceived class prestige predicts academic self-concept. Berlin: Max Planck Institute for Human Development.

Trede, F., \& Higgs, J. (2009). Framing research questions and writing philosophically: The role of framing research questions. In J. Higgs, D. Horsfall, \& S. Grace (Eds.), Writing qualitative research on practice (pp. 195-215). Rotterdam: Sense Publishers.

Tufford, L., \& Newman, P. (2010). Bracketing in qualitative research. Qualitative Social Work, 11(1), 80-96.

U.S. Census Bureau (2010). Economics and statistics administration. Retrieved from http:// census.gov/prod/cen2010/briefs/c2010br-06.pdf

U.S. Department of Education. (2010). Civil rights data collection, 2009-2010: National and state estimations. Retrieved from http://ocrdata.ed.gov/StateNationalEstimations/Projects_2009_10

U.S. Department of Education. (2012). Civil rights data collection, 2011-2012: National and state estimations. Retrieved from https://catalog.data.gov/dataset/civil-rightsdata-collection-201112

U.S. Department of Justice (2006). Bureau department of justice statistics: Prisoners 2005. Retrieved from http://www.bjs.gov/content/pub/pdf/p05.pdf

Uwah, C.J., McMahon, G.H., \& Furlow, C.F. (2008). School belonging, educational 
aspirations, and academic self-efficacy among African American male high school students: Implications for school counselors. Professional School Counseling, 11(5), 296-305.

Valenica, R.R., \& Solóranzo, D.G. (1997). Conceptualizing the notion of deficit thinking. In Valencia, R.R., The evolution of deficit thinking: educational thought and practice (pp. 1-12). New York, NY: Routledgefalmer Taylor \& Francis Group.

Valencia, R.R. (2010). Dismantling contemporary deficit thinking: Education thought and practice. London, England: Routledge.

Van Manen, M. (1997). Researching the lived experience: Human science for an active sensitive pedagogy. London, England: The Althouse Press.

VanTassel-Baska, J., \& Brown, E.F. (2007). Towards best practice: An analysis of the efficacy of curriculum models in gifted education. Gifted Child Quarterly, 51(4), 342-358.

VanTassel-Baska, J., Feng, A. X., \& Evans, B. R. (2007). Patterns of identification and performance among gifted students identified through performance tasks: A threeyear analysis. Gifted Child Quarterly, 51(1), 218-231.

Vargas, G.R. (2003). The (racially neutral) politics of education: A critical race theory perspective. Educational Administration Quarterly, 39(1), 68-94.

Viotti da Costa, E. (2001). New publics, new politics, new histories: From Economic reductionism to cultural reductionism. Reclaiming the Political in Latin American History: Essays from North America. Gilbert M. Jones (ed.). Durham and London: Duke University Press.

Vogl, K., \& Preckel, F. (2014). Full-time ability grouping of gifted students: Impacts on 
social self-concept and school related attitudes. Gifted Child Quarterly, 58(1), 1568.

Walton, G. M., \& Cohen, G. L. (2007). A question of belonging: Race, social fit, and achievement. Journal of Personality and Social Psychology, 92(1), 82-96.

Walton, G. M., \& Cohen, G. L. (2011). A brief social-belonging intervention improves academic and health outcomes of minority students. Science, 33(1), 1447-1451. doi:10.1126/science.1198364

Webb, P., \& Linn, C. (2016). Acting white or acting right: Exploring racial identity at an HBU. Journal of Black Studies, 47(2), 134-149.

Weiner, L. (2006). Challenging deficit thinking. Educational Leadership, 64(1), 42-46.

Wells, R., Lohman, D., \& Marron, M. (2009). What factors are associated with grade acceleration? An analysis and comparison of two U.S. databases. Journal of Advanced Academics, 20(1), 248-273.

Westberg, K.L., \& Daoust, M.E. (2004). The results of the replication of the classroom practices survey replication in two states. Storrs, CT: National Research Center on the Gifted and Talented, University of Connecticut.

Whiting, G. W. (2006). Promoting a scholar identity among African-American males: Recommendations for gifted education. Gifted Education Press Quarterly, 20(3), 2-6.

Whiting, G.W. (2009a). Gifted black males: Understanding and decreasing barriers to achievement and identity. Roeper Review, 31(1), 224-233.

Whiting, G. W. (2009b). The Scholar Identity Institute: Guiding Darnel and other Black males. Gifted Child Today, 3(1), 53-58. 
Whiting, G. W. (2010). Overrepresentation of African American males in special education: A clarion call for action and change. In E. M. Zamani-Gallaher \& V. C. Polite (Eds.), The State of the African American Male, (pp. 19-44). East Lansing, MI: Michigan State University.

Wiggan, G. (2007). From opposition to engagement: Lessons from high achieving African American students. The Urban Review, 40(4), 317-349.

Williams, Carrie B. (2007). Research methods. Journal of Business and Economic Research, 5(3), 65-72.

Wilson, T.D. (2002). Alfred Schutz: Phenomenology and research methodology for information behavior research. The New Review of Information Behavior Research, 3(1), 71-81.

Wimpenny, P. (2000). Interviewing in phenomenology and grounded theory: Is there a difference?. Journal of Advanced Nursing, 31(6), 1485-1492).

Winsler, A., Karkhanis, D.G., Kim,Y.K., \& Levitt, J. (2013). Being black, male, and gifted in Miami: Prevalence and predictors of placement in elementary school gifted education programs. Urban Review, 45(4), 416-447.

Wright, B.L., Ford, D.Y., \& Young, J.L. (2017). Ignorance or indifference? Seeking excellence and equity for under-represented students of color in gifted education. Global Education Review, 4(1), 45-60.

Yin, R.K. (2003). Case study research design and methods ( ${ }^{\text {rd }}$ ed.) Thousand Oaks, CA: Sage Publications. 


\section{APPENDICES}

APPENDIX A

\section{INFORMED CONSENT}

Present But Not Accounted For: A Phenomenological Study of Gifted African American Males

Principal Investigator:

Kyle W. Ingle

College of Education and Human Development

University of Louisville

1905 South $1^{\text {st }}$ Street

Louisville, KY 40292

wking101@lousville.edu

Ronda E. George

University of Louisville

1905 South $1^{\text {st }}$ Street

Louisville, KY 40292

rescru01@cardmail.louisville.edu

Site where study is to be conducted: * Middle School

Phone number for subjects to call for questions:

\section{Introduction and Background Information}

My name is Ronda George and I am conducting research at __ Middle School as a doctoral candidate from the University of Louisville's College of Educational Leadership and Organizational Development under the direction of the Principal Investigator, Kyle W. Ingle, Ph.D., University of Louisville. My research explores the topic of the lived experiences of gifted African American males in grades 6-8. Eleven subjects will be invited to participate in this study.

\section{Purpose}

The purpose of the study is to better understand the experiences of gifted African American males who participate in a gifted program. The study will also exam how gifted African American males perceive their sense of self-efficacy and the extent to which self-efficacy influences their academic persistence. Information from this study can help in reformulating gifted assessment instrumentation, identification criteria, 
enrollment, and retention practices that have historically contributed to the underrepresentation of African American males in gifted programs. The data collected is intended to provide insight to schools, school districts, and policymakers as a means to not only increase enrollment in gifted programs, but to improve program quality and enhance their academic and social experiences within the gifted program.

\section{Procedures}

Each participant will be asked to participate in a 45-60-minute interview consisting of 11 questions constructed to encourage the participant to think thoughtfully about their daily experiences as a student in a gifted program. The participant will have the option of identifying themselves or they may remain anonymous. The interview is confidential and recordings and transcripts will be locked in a file cabinet for security purposes. The interview recordings and transcript will only be accessible to me as the researcher. Participant may decline to answer any question that makes them feel uncomfortable. Participation is completely voluntary and participants may withdraw at any time without consequences.

After all interviews have been completed all of the participants will be invited to participate in a focus group. The purpose of the focus group is to gather more in-depth data which can be derived from the interactive sharing among a group of gifted African American males. Participation in the focus group will also be completely voluntary.

\section{Potential Risks}

There are no foreseeable risks other than possible discomfort in answering personal questions. Although there are no foreseeable risks, there may be unforeseen risks.

\section{Benefits}

The participant's contribution in sharing experiences and perceptions about their educational experiences as gifted African American males is expected to provide insight to schools and school districts about the most effective ways to provide academic and social supports for this student population. It is also expected that the findings of the study will influence the inclusivity and cultural sensitivity in the construction and implementation of future practices and policies that govern the identification, recruitment, and retention of gifted African American males to gifted programs.

\section{Payment}

Participants will not be compensated, but will be given a $\$ 10$ gift card in appreciation for their contribution to the study.

\section{Confidentiality}

Total privacy cannot be guaranteed. We will protect participant privacy to the extent permitted by law. If the results from this study are published, participant names will not 
be made public. Once participant information leaves our institution, we cannot promise that others will keep it private.

Participant information may be shared with the following:

- The University of Louisville Institutional Review Board, Human Subjects Protection Program Office, Privacy Office, others involved in research administration and compliance at the University, and others contracted by the University for ensuring human subjects safety or research compliance

- People who are responsible for research, compliance and HIPAA oversight at the institutions where the research is conducted

- Government agencies, such as:

o Office for Human Research Protections

o Office of Civil Rights

OR: Your identity as a subject in this study and the information you provide may be released and published (only if the participant agrees that the information may be made public).

\section{Security}

Interview and focus group recordings and transcriptions will be kept private by being kept in a locked file cabinet. Electronic research manuscripts will only be accessible using a password protected computer.

\section{Voluntary Participation}

Taking part in this study is voluntary. Participants may choose not to take part at all. If participants decide to be in this study, they may stop taking part at any time. If the participant decides not to be in this study or if the participant stops taking part at any time, they will not lose any benefits for which they may qualify.

\section{Contact Person}

If you have any questions or concerns about the study, please do not hesitate to contact the researcher, Ronda George at rescru01@cardmail.louisville.edu

\section{Research Subject's Rights}

If you have any questions about participant rights, you may call the Human Subjects Protection Program Office at (502) 852-5188. You may discuss any questions in private, with a member of the Institutional Review Board (IRB). The IRB is an independent committee made up of people from the University community, staff of the institutions, as well as people from the community not connected with these institutions. The IRB has approved the participation of human subjects in this research study. 


\section{Concerns and Complaints}

If you have concerns or complaints about the research or research staff and you do not wish to give your name, you may call the toll free number 1-877-852-1167. This is a 24 hour hot line answered by people who do not work at the University of Louisville.

This informed consent document is not a contract. This document tells you what will happen during the study if the subject chooses to take part. Your signature indicates that this study has been explained to you, that your questions have been answered, and that you give consent for your child to participate in the study. You are not giving up any legal rights to which you are entitled by signing this informed consent document. You will be given a copy of this consent form to keep for your records.

Subject Name (Please Print)

Signed

Printed Name of Legally

Signed

Authorized Representative (if applicable)

Signature of Legally

Date

Authorized Representative

Authority of Legally Authorized Representative to act on behalf of Subject

*Authority to act on behalf of another includes, but is not limited to parent, guardian, or durable power of attorney for health care.

Printed Name of Person Explaining Consent Form Signed

Consent Form (if other than the Investigator) 


\section{APPENDIX B}

\section{ASSENT FORM}

Student's Name

You are being asked to participate in a study that will help teachers and other staff understand your views of yourself and your experiences as a gifted African American male in a gifted program. Your role in this study will be to participate in an individual 45-60 minute interview. You will also be invited to participate in a focus group. During the focus group, you and other participants will have the opportunity to respond to prompts and engage with each other for approximately an hour and a half. Your responses in the interview and focus group are confidential, which means that your answers and/or comments will only be shared with the researcher and no one else. The purpose of this study is to provide insight to help schools and school districts improve the educational experiences of gifted African American males. You may choose to withdraw at any time without any consequences.

Your parent/guardian has approved your participation in the interview and focus group. The interviews and focus group will be scheduled within two weeks of each other. Questions asked during the interview or focus group are not a test and there is no wrong answer. Your participation is greatly appreciated and as an incentive for participating, after completing both the interview and focus group, you will be given a $\$ 10$ gift card. By signing below, you are agreeing to participate in the study.

Student Signature

Date

I understand that my participation in this study is completely voluntary and I may withdraw at any time without consequence by contacting the researcher using the contact information listed below. I understand that unless I agree, my name will not be associated with responses or participation.

If you have any questions or concerns about the study, please do not hesitate to contact the researcher, Ronda George at rescru01@cardmail.louisville.edu. If you have questions 
about your rights as a participant, contact the Human Subjects Office at the University of Louisville at (502)-852-5188. 


\section{APPENDIX C \\ INTERVIEW QUESTIONS}

1. Tell me about yourself and your school experiences.

2. What have your experiences been as a participant in a gifted program?

3. What does being gifted mean to you?

4. What makes you feel accepted or not accepted in this group?

5. Have you ever experienced discrimination in your school environment? Describe the situation and how you responded to it.

6. Have you ever wanted to be exited from the gifted program? Describe the circumstances that made you feel that way. If you have never wanted to be exited, what experiences make you want to continue?

7. Why do you think you can be successful in gifted program? Give me a time when you bounced back.

8. Describe an experience in your class that made you feel your ethnicity was valued.

9. Describe your support system in school and outside of school.

10. How do you perceive your academic achievement in comparison to your peers? 


\section{APPENDIX D}

\section{FOCUS GROUP PROTOCOL}

\section{Research Questions}

3. How do gifted African American males understand and experience participation in a gifted program?

4. How do African American males perceive their participation in a gifted program as shaping their sense of self- efficacy and academic persistence?

\section{Materials Needed}

- Approximately 6 chairs arranged in a circle

- Table to arrange snacks

- Tables for drawing

- Snacks for 7 people (drink, chips/cookies, cups, napkins, plates)

- $\quad$ Playlist set up (for music to play while participants are arriving)

- About 7 nametags

- Sharpies to write on chart paper

- Focus group tape recording equipment

- 12 \$10 gift certificates

- Small alarm clock (digital) for facilitator to glance at

- Plain white paper

- Pencils

- Focus group expectations on flipchart

- Introductions and Icebreaker on flipchart

- Masking tape

- 1 clipboard and legal pad

\section{Focus Group Site Preparation}

1. Arrange chairs in circular formation for at least 6 participants.

2. Setup snacks for at least 6 people. 
3. Have name tags and black magic markers available for participants to write their first names only.

4. Place plain white paper on tables.

5. Test recording equipment and place strategically for optimal recording.

6. Greet participants as they enter the room. Encourage them to take some snacks.

7. Ask participants to turn off cell phones.

8. Ask participants to use the restroom before the focus group gets started.

\section{Introduction}

\section{Welcome and Facilitator Introduction}

"Good afternoon and welcome to our discussion. Thanks for taking the time to join me in a discussion about the experiences of gifted African American males who participate in a gifted program or magnet. My name is Ms. Ronda George and I will be the facilitator for the focus group discussion today.

\section{Background on PCC Bridge Project and Purpose of Focus Group}

"I'm going to tell you a little bit about my study and what you can expect today. For the last two and a half years I have been studying about gifted African American males and what other researchers have reported about how they understand and experience school. I now want to talk to the real experts, you, to find out about your experiences and about how you feel and think about the environment in which you learn each day and about you as individuals. I am going to use what I learn from you today and from to help me inform school and school districts about how they can best serve gifted African American males based on what we have learned about their experiences.”

"Our focus group discussion is going to last about an hour and half. Focus groups are different from workshops or classes at school. Once we get started, you are going to give your reactions to some photographs I will show you. I am going to ask you a few questions and you are going to share your thoughts and opinions. You will do most of the talking. I will be doing a lot of listening. Remember I want to learn from you.”

\section{Appreciation}

"To show my appreciation for what you will teach me and for your time, I have \$10 gift certificates that I will give to each of you at the end of the session."

\section{How Today's Focus Group will work}

1. No "Right" or "Wrong" Answers and Participation"I will be showing you four different photographs, one at a time. I will you give you a few moments to fully take in what you are seeing. I will then ask you to call out words or phrases that come to your mind that reflect your thoughts on 
what you see and how it makes you feel or what the photo represents or means to you. This is called free listing. I will write all of your responses on the chart paper. Remember, these are your thoughts, there are no right or wrong answers. We will do this for all four photographs.

After we have exhausted our lists, I'll be asking you a few questions that relate to the photographs and some of the thoughts you listed. Again, I want you to know that there are no "right" or "wrong" answers, and it's okay to have a different opinion from other people in the group. It's really important for us to hear all the different points of view in the room. I want you to share your point of view, even if it is different from what others are saying, and I want you all to respect each other's opinions. Please don't make fun of what other people say or argue with them."

"I also don't want you to feel like you have to respond to me all the time. Feel free to talk to each other when discussing my questions. If you want to respond to something someone said, or if you want to agree or disagree, or give an example, you can do that, just be respectful. We want all people to have a chance to share ideas. We may need to interrupt or call on people to make sure this happens. Please do not feel offended if we do this. I will let you know when we are ready to move on to the next question."

\section{Recording and Confidentiality}

"Before we get started, I want to remind you that I will be digitally recording the session because we don't want to miss any of your comments. People often say things in these sessions, and I can't write fast enough to write them all down."

"Although will use each other's first names today, I will not use any names in my study unless you say it is OK. You can be assured of complete confidentiality. No one will be able to link your name back to what you said I am the only one who will be listening to the recording. I am also going to ask all of you to keep what is said here confidential, so that everybody feels comfortable talking and knows what they say will not be repeated. Can you all do that?" (Make eye contact with each person in the group and wait for them to nod affirmatively.)

"You do not have to answer any question that makes you feel uncomfortable."

\section{Timing, Survey and Gift Certificates}

"We expect to be here until . We appreciate you giving us your time, and we want to make sure we end on time. Lori will be watching the clock and may need to interrupt the discussion at times and move us on to another question to be sure we have time to discuss all topics. Lori may also ask you some questions, give a summary or remind me of something I missed."

"At about 3:50, we will end the focus group discussion. Before you are dismissed, I will distribute a $\$ 10$ gift certificate to each of you as a token of our appreciation 
for your time and participation."

(Tell the group that you will be starting the tape recorder and do so.)

\section{ICEBREAKER}

(5 minutes)

1. "Let's begin. I have asked you to wear a name tag to help me remember each of your names. Let's go around the circle and introduce ourselves. Please give me your first name and age, and just for fun, tell us what you enjoy doing most and why. I'll start...”

\section{FREE LISTING}

1. There are four images around the room. Take a moment to look at them carefully. What do you think of when you see the images? How do they make you feel? Can you identify with any of them? What is positive or negative about the images?

2. After participants have viewed the photographs, ask them for words or phrases to record on the chart paper to create the free lists.

\section{MAIN QUESTIONS}

(60 minutes)

1. Which of the images did you identify with most?

2. Did any of the photos cause you to have specific memories of your experiences in school?

3. What do think the character in the picture might be thinking?

4. How did viewing the photographs make you feel?

\section{WRAP UP QUESTION}

1. If you could speak to any of the characters in the photographs, what would you say to them and why?

\section{PARTICPANT DRAWINGS}

After the discussion and free list have been exhausted. Participants will generate an image of their own.

"Now I would like for you to create a picture of your own. You do not have to be an artist. Everyone can participate. I would like for you to draw a picture that represents your schooling experiences. You can also use symbols or text to enhance your drawing. There is not right or wrong way to do this. The drawing should represent you, your experiences of your desires concerning your education and schooling experiences in general. 


\section{GIFT CERTIFICTATES}

(2-5 minutes)

Give each youth a gift certificate. 


\section{APPENDIX E}

\section{PHOTO ELICITATION IMAGES}

\section{Figure E1}

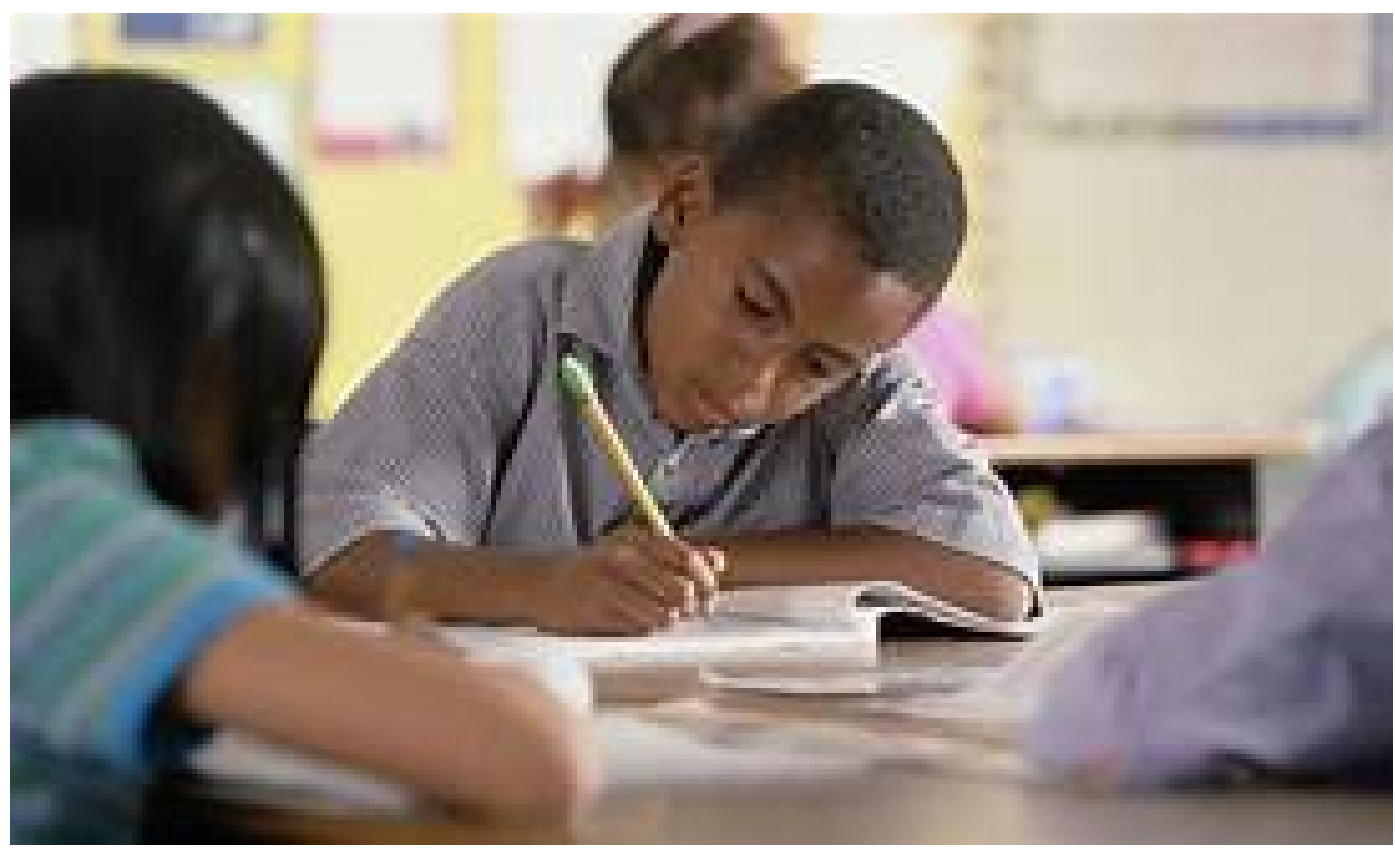

Figure E2

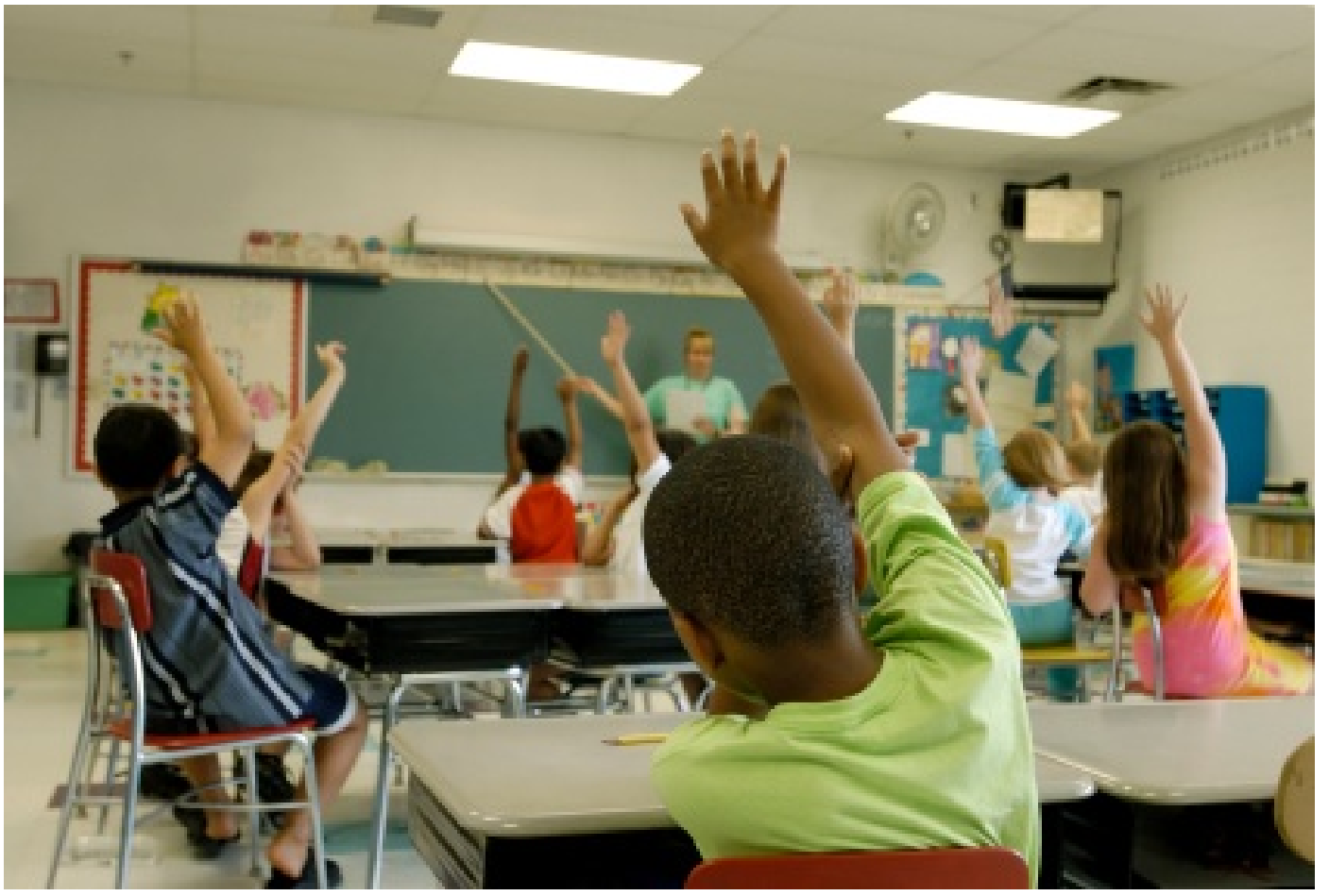


Figure E3

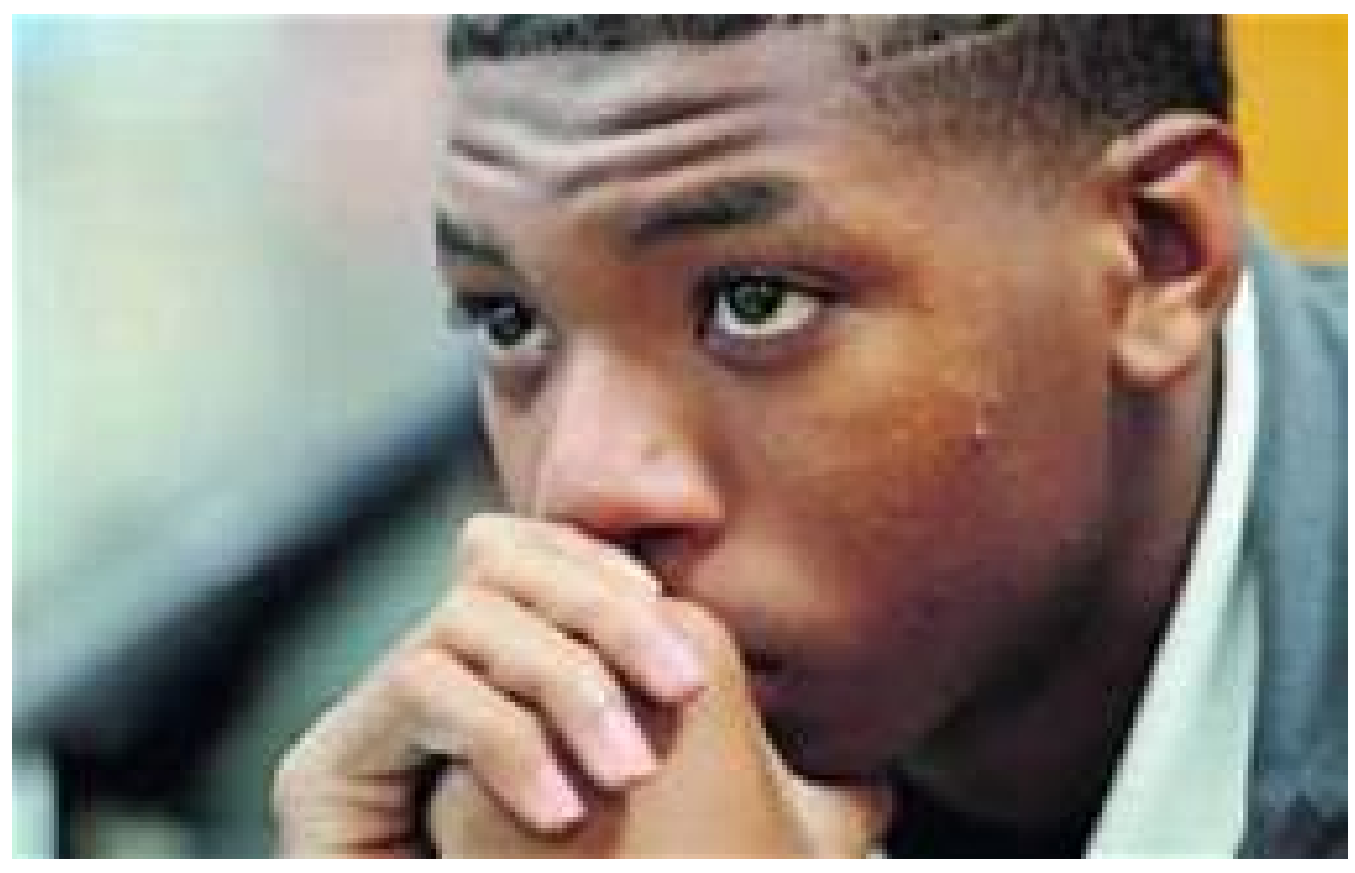

Figure E4

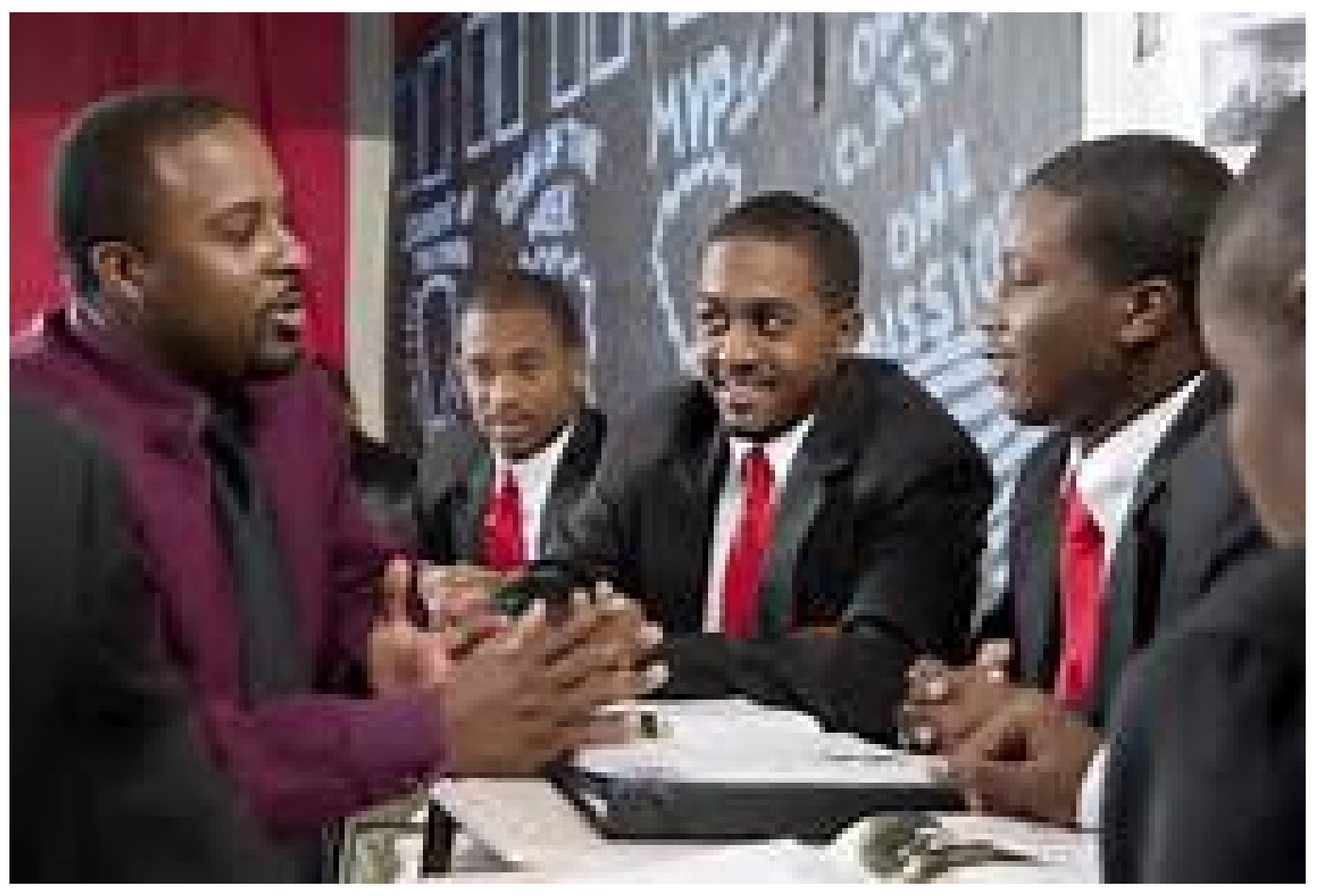




\section{RESEARCHER POSITIONALITY}

Dillard (2000) purported that historically, people of color have been demoralized, repressed, and their contributions undervalued in education research. As a woman of color having to contend with the effects of pervasive and enduring cultural, economic, and educational inequality has served as the impetus for pursuing an interpretive phenomenological study on gifted African American males. The purpose of the study is to contribute to the body of knowledge on the experiences of gifted African American males in grades 6-8. The study also expands upon prior knowledge and analyses of gifted African American males, providing a platform for their voices to be heard from their experiences as a middle school student in a gifted program. As such, my role as the researcher will be examined through exploring the following aspects of positionality: (a) how I personally situate myself in the research process, (b) my knowledge of self in relation to others and (c) exploring the transition from self-examination to an examination of systems.

\section{Researching the Self}

"And as the mother forgets her agonies in the bliss of clasping her babe to her breast, so the bent and heart-sore immigrant forgets exile and homesickness and ridicule and loss and estrangement, when he beholds his sons and daughters moving as Americans among Americans” (Antin, 1912, p. 272).

As a Black female educator and researcher, my experiences and understanding of the world have implications for my methodological approach to research, the manner in which I collect and interpret data, and the ways in which I conceptualize my interactions with research subjects. My epistemological beliefs are shaped through the historical 
legacy and present-day realities of racial injustice, cultural dismissiveness, and deficit ideological thinking experienced by people of color in the United States. While I have committed to ensuring the fidelity of the research process and moderating for credibility, dependability, and trustworthiness, my analytic interpretations of the research data are not void of the influences of my knowledge of self as it relates to my cultural background, gender, and belief system. In fact, Milner (2007) asserted that in studying individuals and groups of people of color, in order to avoid deleterious representation of the subjects, researchers should be cognizant of their positionality on race and culture. As such, transparent introspection on who I am as an individual in the position of researcher, holds me accountable for delivering an unblemished narrative of the lived experiences of gifted African American males that begins with a narrative of self.

I vividly recall our first day back from Thanksgiving break. I sat attentively in the 7th/8th split accelerated Language Arts class in I.S. 231 waiting for the teacher to give instructions for the opening activity. There were many Black faces, but I knew there was distance between them and me that I could not explain at that time. I clearly understood my differences from the Hispanic, Indian, and Asian students in the overcrowded New York City classroom, but I felt like an imposter of sorts. I looked like the other Black kids, but I was different. Just as expected, the teacher asked us to share with the class what favorite foods we enjoyed for Thanksgiving. From various corners of the room, the other Black students yelled out macaroni and cheese, candied yams, cornbread, and deviled eggs. I felt as though my family must have gotten Thanksgiving all wrong, because we did not have any of those foods at our table. My Caribbean parents prepared native foods for Thanksgiving such as rice and peas, curried goat, and fried plantains. I 
loved those foods and had a wonderful Thanksgiving, but in an attempt to fit in and to avoid the scrutiny and interrogation of my Black peers, I followed the lead of a classmate and claimed I had macaroni and cheese too. A very simplistic memory, but profound in its shaping of my sense of cultural acceptance, belonging, and identity formation.

My parents immigrated to the United States in the 1960's. My parents were hardworking and I was raised in a middle-class neighborhood in Queens, NY. As a child, I was oblivious to the pathos of racism that had permeated this country I called home. I have no recollection of discussions on the conditions of race relations in theU.S.in our home until I was much older. In hindsight, I realize it was probably because my parents themselves were trying to make sense of the impact of the Vietnam War on the United States, Civil Rights protests, the assassinations of President John F. Kennedy and Martin Luther King Jr., and the Cuban Missile Crisis and how those events would shape their experiences. For many it is inconceivable to imagine the enormity of leaving one's native land and being thrust into one of the most volatile era's in a nation's history. They found comfort in socializing and fellowshipping with other West Indians in our neighborhood and church. The diversity in New York allowed them to have access to food from the islands, which I'm certain served to soften their periodic longing for the comforts of home. Despite their longing, my parents came to the United States to evade the clutches of poverty and pursue the American dream.

Establishing a new home in a new country would prove to be challenging. My parents advanced in their social mobility through schooling and obtaining higher paying jobs. The commitment to hard work was not without reward. They soon began to experience racism, discrimination, and being ostracized and ridiculed. They were at 
times the butt of jokes about foreigners, and painfully, many of the jokes came from African Americans who could not detect my dad's Trinidadian accent as a result of his many hours of practice for broadcasting. He sounded like an American. They would complain to him about all of the foreigners “coming over here and taking our jobs”. Little did they know, they were speaking to the culprit. In fact, I have personally encountered this kind of conversation many times. They would make fun of West Indians having many jobs and saving money to bring other family members to the states. As I got older my parents would have discreet discussions about the discrimination they experienced as we sat together for dinner. These conversations I did remember and may account for the reason I disassociated my ethnic background in an attempt to camouflage my experiences to reflect that of other African Americans. I didn’t want to be different. I didn’t want to be the butt of a joke. I wanted to be $100 \%$ American like them.

The significance of the Thanksgiving memory can be expanded upon through the lens of Rumbaut's (1994) seminal work on the children of immigrants. Rumbaut's study examined the degree to which the formation of ethnic self-identities during adolescence influenced the psychosocial adaptation of the children of immigrants from Asia, Latin America, and the Caribbean. While the findings indicated that there were significant differences among ethnic self-identification between and within ethnic groups, patterns emerged from the multivariate analysis indicating common factors shaping the process of ethnic self-identification and other aspects of cognitive and affective psychosocial adaptation. Variables such as gender, age, nativity, socio-economic status, parent-child relationships, language proficiency, educational attainment, and experiences with discrimination were examined as predictors of depression and self-esteem. 
Rumbaut found that a) issues of gender are strongly associated with self-identity; b) acculturation affects the identity process in assimilation; c) psychosocial adaptation is cast by family dynamics; and d) cognizance of discrimination affects how children define their ethnic identity. Understanding the intersection of these factors in relation to the psychosocial outcomes for the children of immigrants confirms Rumbaut's assertion that "youth see and compare themselves in relation to those around them, based on their social similarity or dissimilarity with the reference groups that most directly affect their experiences” (p. 754). Furthermore, this assertion validates the feeling of cultural dissonance I experienced as an adolescent. As a researcher who is a minority within a minority, investigating the lived experiences of gifted African American males, I am well acquainted with the schism they encounter between intelligence, ethnicity, and gender and the struggle to be accepted in your peer group.

My experiences as an African American female student, in an advanced program, in many ways mirrors the experiences described in the literature on the experiences of gifted African American males. Drawing upon Rumbaut's observations of the manner in which adolescents compare themselves to those around them, I bring to the research process, the understanding that the development of racial self-identity is paramount to African American students successfully negotiating the pre-existing racialized construct of societal agencies that limit access to equal opportunity. As an example, if ethnic images are underrepresented in gifted programs and African American males find no relevance or connection to the social context of a gifted program, gifted African American males may disavow their giftedness in search of an environment where their concept of self and socioemotional well is not challenged or undermined. As a 
researcher, I can derive meaningful interpretations from the narratives of the study's participants from racial and cultural experiential similarities. The acknowledgement of racial and cultural similarities however, does not negate the importance of giving attention to cultural differences that may exist in instances where the researcher and participant are from the same ethnic group. In fact, realizing within group differences provides an opportunity for me as the researcher to confront my own biases and preconceptions about the subjects under investigation.

My adolescent experiences were the genesis of a journey which has propelled me to amplify the voices of adolescents who have been marginalized. Hence, the phenomenological approach to my research will afford me the opportunity to publicize the unique abilities of African American males that have gone unnoticed. Making meaning from the lived experiences of the subjects, invites me to be reflective of the ways in which I have made meaning from my lived experiences and how the evolution of those meanings governs and influences my position and disposition as a researcher. As a result, exposing the inequities that have led to the underrepresentation of African American males in gifted programs, and challenging policies and practices that limit access to quality gifted programming serves as my contribution to and participation in a social justice imperative for males of color.

\section{Researching the Self in Relation to Others}

“...we are all members of cultural communities where the interpretation of our life experiences is mediated by the interaction of a complex set of variables, such as gender, social class, age, political affiliation, religion, and region (Banks, 1998, p. 5). 
My exploration of self and finding meaning in the world as an Black female was the driving force behind my decision to become an educator. I have lamented over the persistent underachievement of African American students over the course of my twentyone years as an educator in urban schools, particularly gifted African American males. My personal encounters with gifted African American males certainly confirmed their academic aptitude, yet there loomed a cloud of despair because their academic strengths were rarely realized in classrooms and in the state's system of accountability. Furthermore, in my own school it was clear that gifted African American males went unidentified and/or experienced varying degrees of instructional rigor, low expectations from teachers, and had novice teachers who were not prepared to teach gifted African American males amidst the unique challenges of an urban school setting.

My passion and drive for my students fueled a great sense of urgency within me. This urgency led me to question what was happening with instructional practices, and the culture and climate in classrooms all over the country that has gripped the academic achievement of African American students with such fierceness, that their academic performance continued to lag behind that of their White counterparts. On many occasions I engaged in discussions with colleagues and school leaders about barriers to achievement and culturally responsive teaching strategies designed to meet the learning needs of children of color, in hopes of reversing the historical narrative of their educational deficiencies. Admittedly, the constant stream of devitalizing dialogue on the performance of African American students aroused a sense of despair. I started to succumb to negative rhetoric and finger-pointing as a result of my inability to bring more instantaneous resolution to this daunting predicament. However, as a reflective educator 
and lifelong learner, I am aware that there is an ongoing quest to equitably and efficaciously educate gifted African American males.

African American males are more likely than other student groups to be identified as being a behavior problem and their academic achievement is often characterized as being at risk, particularly as it relates to math and reading test scores, low graduation rates, disproportionate referrals to special education, and high dropout rates (Boykin \& Noguera, 2011). In my role as a teacher, I assessed that African American students experiencing a deficit-based educational experience lacked academic confidence and overtime became socially and academically displaced. I would often have one-on-one conversations with my students of color, as if to give them an infusion of hope when I saw them bending under the weight of apathy. My admonishment would always begin with 'tell me what's going on with you, I know you can do better than this'. This was my strategy for getting students to divulge their innermost thoughts about their school experiences, realizing that if I could help them identify the root of the problem, the academics would take care of themselves. My students would consistently tell me-`I hate this school, these teachers don’t care, I don’t want to be here. My translation of this-I don't feel like I belong here, teachers don’t know me or care about me as a person, and I don't want to be somewhere where I am misunderstood, devalued, and feel like I am treated differently than other students. Along the same lines, Delgado Bernal (2002) found that "although students of color are creators of knowledge, they often feel as if their histories, experiences, cultures, and languages, are devalued, misinterpreted, and omitted within formal educational settings” (p. 106). As such, my anecdotal 
interpretations prompted me to see the world through their eyes and question how the role of others constructed their perceived realities.

The student's educational realities gave momentum to perpetuating an attitude of indifference within the school context. Their negative approach to being a student was in some instances exacerbated by issues of not having access to or taking advantage of resources needed to be successful in school, low parental involvement, and the negativity projected on them from their parents who also harbored animosity about their unfavorable educational experiences. Growing up in a two-parent, middle-class household insulated me from the kinds of struggles my student's experienced, however in my experience as an educator, I have encountered many students struggling to navigate insurmountable barriers, giving me a strong sense of how their social and educational trajectory could easily be adversely affected. To further support this contention, Burke, Sebring, Allensworth, Luppescus, and Eaton (2010) and Payne (2010) opined that if purposeful measures are not taken to avert negative outcomes, dysfunctional school cultures will persist in which student and educator relationships are stunted. Being mindful of the paradigm forming experiences of the research participants, I am intentional about not devaluing their experiences, casting dispersions, or imposing my values system or beliefs when making meaning from their experiences.

\section{Shifting from Self to System}

"In the practice of research, researchers take into consideration, for example, how history and politics shape their racialized and cultural systems of knowing and those of research participants" (Milner, 2007). 
My reflection of self can be contextualized through the systems that legitimize the role of race in society and its subsequent impact on the subjects under investigation in the current study. As a Black female researcher, there is an ever-present consciousness of the undercurrent of race that has permeated the justice system and the education system. Both systems having implications on the social mobility of people of color due to their history of oppression in the United States. Critical Race Theory (CRT) provides a lens to understand the role of race and how it is operationalized within societal systems. CRT scholars explore the ways that supposed race-neutral policies and laws sustain racial, cultural, and gender subservience (Delgado Bernal, 2002). The use of CRT then qualifies the examination of gifted African American males as being a group to which the nuances of culture and ethnicity have been largely ignored and perpetuated through systems where racial subordination is at its core.

The shift from examining self to system became apparent to me as an administrator in the district's only middle school gifted magnet program. Being new to the school, I spent the first year learning the students, staff, and school culture. I was consumed by the managerial tasks that monopolized my day and gave little attention to the systems of inequity that were right under my nose. While observing a lesson in a gifted classroom, I was amazed at the genius present in the room. The engagement was high, the content was rigorous, and the discourse far exceeded any middle school discussion I have ever heard. That particular moment could be described as an administrator's dream observation, but the longer I observed the class, I started to think beyond the lesson, to thinking about how some students got here and others did not. I 
pondered the gross underrepresentation of students of color, especially the males. They simply were not there.

After the observation, I peered into the classroom windows of the other gifted classrooms. I counted aloud to myself to determine how many African American students were in each class of thirty-one students. I counted three of the approximately 124 students on the gifted team. Feeling deflated, I walked back to my office, and tried to make sense of this astonishing reality. Having taught unidentified gifted students of color in my career as a math teacher, I knew these students existed, but were somehow excluded, not just from the classroom, but from a system that seemed to have normalized the underrepresentation of students of color. Continuing to ponder the situation, I realized, I had never even heard the topic of underrepresentation discussed in our administrative team meetings. I had never heard discussion on revisiting the applicant criteria to ensure it was culturally inclusive. I had never heard anyone give thought to how students of color perceive their experiences in a gifted program, although addressing the socio-emotional needs of our gifted students was a high priority. This silence had become deafening and these crucial omissions were evidence of systemic deficits in identifying and sustaining the enrollment of African American students in our gifted program.

Milner (2007) purported that predominant systems working against people of color can taint what we know about them and the way we acquire knowledge about them. When examining educational systems, Ladson-Billings and Tate (1995) aptly suggested that in using CRT to understand the role of race and race-based inequities, access to rigorous curriculum and high-quality programming has primarily benefited White 
students. This was the case in my assessment of the gifted program in my school. While I have seen incremental progress over time, an attempt to level the playing field for students of color, progress may be stymied because based on my experience, there has been minimal evidence pointing to the elimination of social, economic and educational disparities.

Exploring the systems most effecting the subject of this study and the profundity of the role of race within systems, DeCuir and Dixson (2004) asserted that looking through the frame of CRT, "race should be the center of focus and charges researchers to critique school practices and policies that are both overtly and covertly racist” (p. 30). As an ethnic researcher, my study brings to the forefront the imperative of looking at systems that do not provide access to culturally different students. My study aims at challenging accepted norms and interrogating the systemic dominance of the cultural majority, in order to obtain cultural representation in gifted programs reflective of the students within a school community. My success as a researcher hinges on my ability to affect systemic change through policies and practices that produce quantifiable results for gifted African American males. 


\section{REFERENCES}

Antin, M. (1912). Th promised land. New York: Houghton Mifflin.

Banks, J.A. (1998). The lives and values of researchers: Implications for educating citizens in a

multicultural society. Educational Researcher, 27(1), 4-17.

Boykin, A.S., \& Noguera, P. (2011). Creating the opportunity to learn: Moving from research to

practice to close the achievement gap. Washington, DC: ASCD.

Bryk, A.S., Sebring, P.B., Allensworth, E., Luppescu, S., \& Easton, J.Q. (2010).

Organizing

schools for improvement: Lessons from Chicago. Chicago, IL: University of Chicago Press.

DeCuir, J.T., \& Dixsox, A.D. (2004). So when it comes out, they aren't that surprised that it is

there: Using critical race theory as a tool of the analysis of race and racism in education. Educational Researcher, 33(5), 26-31.

Delgado Bernal, D. (2002). Critical race theory, Latino critical race theory, and critical race-

gendered epistemologies: Recognizing students of color as holders and creators of knowledge. Qualitative Inquiry, 8(1), 105-126.

Dillard, C.B. (2000). The substance of things hoped for the evidence of things not seen: 
Examining an endarkened feminist epistemology in educational research and leadership. International Journal of Qualitative Studies in Education, 13(6), 661681.

Ladson-Billings, G., \& Tate, W. (1995). Toward a critical race theory of education. Teachers

College Record, 97(1), 47-68.

Milner IV, H.R. (2007). Race, culture, and researcher positionality: Working through dangers

seen, unseen, and unforeseen. Educational Researcher, 36(7), 388-400.

Rumbaut, R.G. (1994). The crucible within: Ethnic identity, self-esteem, and the segmented assimilation among children of immigrants. International Migration Review, 28(4), 748-794. 


\section{CURRICULUM VITAE}

\section{Ronda E. George ronda.george@jefferson.kyschools.us}

\section{“... a professional who is energetic and \\ enthusiastic...projects a confidence and \\ knowledge base that provides \\ instructional validity... demonstrated \\ excellence in school leadership..." \\ Mary Hardin \\ Former Administrator}

“...business background in technology

was supportive to the use of videos and

computers in the class...She volunteered

for cooperative opportunities in the

media center and helped teachers to

accommodate computers...I recommend

her with the highest regard..."

\section{Professional Profile}

A visionary instructional leader who uses a unique skill set to guide academic achievement outcomes, build staff capacity, and empower teacher leaders to create relevant and rigorous teaching and learning experiences within a school; experienced in the application of $21^{\text {st }}$ century best practices for school improvement and sustainability.

- Doctor of Education in Educational Leadership and Organizational Development.

- Master of Arts in Middle School Education Administration and a Bachelor of Arts in Middle School Education.

- Content area and administrative certifications.

- Committed to the holistic development of students who are college and career ready, ethically conscious and value community reciprocity; creating schools where student achievement is an expectation for all students; nurturing teachers who are passionate, knowledgeable and creative in their approach to teaching all children.

\section{Education and Certifications}

Education

Doctor of Education in Educational Leadership and Organizational Development

University of Louisville, Louisville, KY. 2018

Masters of Middle School Education \& Administration

Western Kentucky University, Bowling Green, KY. 2001

Bachelor of Middle School Education

University of Louisville, Louisville, KY. 1995 
Aidan Delaney
2nd Grade Teacher New York City Schools

"...deeply involved in learning about the educational state-of-the-art, investigating research and designing instructional materials...I look forward to the time when Diane will bring her love of children, enthusiasm, initiative, and intelligence into her own classroom." Monica Brink, Ed.D.

"My ability to motivate students and share a love of learning fosters a successful classroom environment. ...I would welcome becoming part of 'the village that raises the child' in your district." Jenny Lysaker

\section{Certifications}

Professional Certificate for Instructional Leadership-Principal-All Grades.

Provisional Certificate for Teaching in the Middle Grades 5-8: Mathematics\& Spanish.

Professional Growth \& Effectiveness System certification.

ELEOT Certified (Effective Learning Environment Observation Tool).

\section{Honors, Organizations \& Leadership \\ Jefferson County Public Schools Deeper Learning Symposium}

Presenter: Culturally Responsive Pedagogy 2017

Jefferson County Public Schools Educator of Color Nominee, May 2017

NISL (National Institute for School Leaders), Aug. 2014-June 2015

AdvancEd External Audit Review Team, October 2014

Jefferson County Public Schools Assistant Principal of the Year 2013

Jefferson County Public Schools F.A.M.E Mentor 2006 \& 2008

Jefferson County Assistant Principals Association Secretary 2008-2009

KLA (Kentucky Leadership Academy) 2004-2006

\section{Key Qualifications}

- Evaluation and supervision of certified and instructional staff.

- Oversight of extracurricular and special programs (academic/non-athletic)

- Instructional coach for teachers in the area of using a wide variety of instructional strategies as well as motivational and implementation strategies to engage students in active learning.

\section{Employment}

Samuel V. Noe Middle

Jefferson County Public Schools, Louisville, KY Assistant Principal 
October 2008-Present

Thomas Jefferson Middle School

Jefferson County Public Schools, Louisville, KY

Assistant Principal

June 2002- October 2008

Thomas Jefferson Middle School

Jefferson County Public Schools, Louisville, KY

Teacher

August 1996-May 2002

Professional Affiliations

Jefferson County Assistant Principal's Association 